Florida International University FIU Digital Commons

\title{
Nanofabrication and Spectroscopy of Magnetic Nanostructures Using a Focused Ion Beam
}

\author{
Ali Hadjikhani \\ FIU, ahadj001@fiu.edu
}

DOI: $10.25148 /$ etd.FIDC000785

Follow this and additional works at: https://digitalcommons.fiu.edu/etd

Part of the Biological Factors Commons, Biology and Biomimetic Materials Commons, Biomedical Devices and Instrumentation Commons, Chemical and Pharmacologic Phenomena Commons, Circulatory and Respiratory Physiology Commons, Electrical and Electronics Commons, Electromagnetics and Photonics Commons, Electronic Devices and Semiconductor Manufacturing Commons, Medical Immunology Commons, Nanotechnology Fabrication Commons, and the Semiconductor and Optical Materials Commons

\section{Recommended Citation}

Hadjikhani, Ali, "Nanofabrication and Spectroscopy of Magnetic Nanostructures Using a Focused Ion Beam" (2016). FIU Electronic Theses and Dissertations. 2536.

https://digitalcommons.fiu.edu/etd/2536 


\section{FLORIDA INTERNATIONAL UNIVERSITY}

Miami, Florida

NANOFABRICATION AND SPECTROSCOPY OF MAGNETIC NANOSTRACTURES USING A FOCUSED ION BEAM

A dissertation submitted in partial fulfillment of the requirements for the degree of DOCTOR OF PHILOSOPHY

in

\section{ELECTRICAL ENGINEERING}

by

Ali Hadjikhani

2016 
To: Interim Dean Ranu Jung

College of Engineering and Computing

This dissertation, written by Ali Hadjikhani, and entitled Nanofabrication and Spectroscopy of Magnetic Nanostractures Using a Focused Ion Beam, having been approved in respect to style and intellectual content, is referred to you for judgment.

We have read this dissertation and recommend that it be approved.

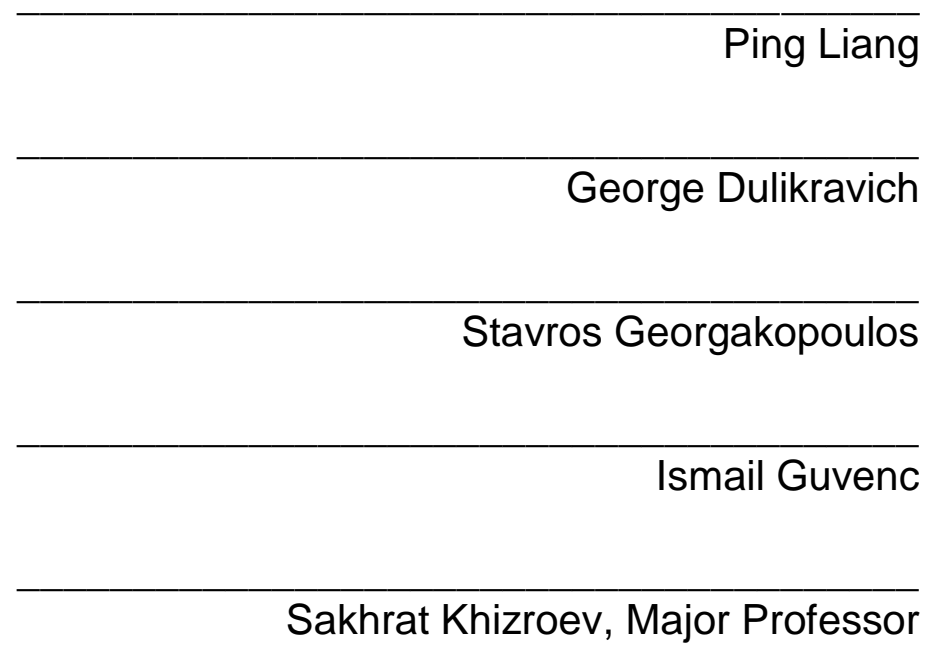

Date of Defense: July 08, 2016

The dissertation of Ali Hadjikhani is approved.

Interim Dean Ranu Jung College of Engineering and Computing

Andrés G. Gil Vice President for Research and Economic Development and Dean of the University Graduate School

Florida International University, 2016 


\section{DEDICATION}

I dedicate this dissertation to my family and my grandparents who have always been there for me 


\section{ACKNOWLEDGMENTS}

It would have not been possible for me to get here without the endless support and help of many people. This dissertation became a reality only with the support of these individuals. I would like to extend my sincere thanks to my advisor, Dr. Sakhrat Khizroev, for believing in me and letting me take the lead. He was always insightful and motivated and this kept me going on as well. I would also like to thank Dr. George Dulikravich, Dr. Ismail Guvenc, Dr. Stavros Georgakapoulos and Dr. Ping Liang, my committee members, for their critical guidance and mentorship. I am grateful to my parents who made me who I am, also my grandparents, and my uncle, Dariush Nami, who were there for me whenever I needed them without even having to ask.

I am thankful to Dr. Arvind Agarwal and Dr. Cesar Levy for all their dedication and support. I would sincerely like to thank Dr. Jeffrey Bokor, Dr. Paul Lum and Dr. Frances Allen at University of California Berkeley for their help and guidance. I have to thank Mark Stone, Brayan Navarrete, Ping Wang and Emmanuel Stimphil for fabricating and characterizing the samples. I sincerely thank Alexandra Rodzinskaia for preparing the bio-samples. She also helped me with every step of writing this dissertation and was more of a friend than a colleague. I have to express my gratitude to Dr. Yusuf Emirov for patiently helping us with our TEM analysis, also Dr. Alex Franco and all the Advanced Materials Engineering Research Institute (AMERI) staff. I would also have to thank Steven Stalbaum and John Barchi for teaching me and mentoring me. Dr. Nouchine Hadjikhani and Dr. Pegah Khoshpouri for helping me to have a better understanding of the bio-distribution data. This

note of gratitude will not be complete without mentioning my friends Ali Karbasi and Roozbeh Nikkhah and also my brother and friend Mehdi Hadjikhani and Sadegh Behdad. Sadegh helped me out as much as my brother did and no matter what the problem, I always knew I could count on him. 


\title{
ABSTRACT OF THE DISSERTATION
}

\section{NANOFABRICATION AND SPECTROSCOPY OF MAGNETIC NANOSTRACTURES}

USING A FOCUSED ION BEAM

\author{
by
}

\author{
Ali Hadjikhani \\ Florida International University, 2016 \\ Miami, Florida \\ Professor Sakhrat Khizroev, Major Professor
}

This research used a focused ion beam in order to fabricate record small nano-magnetic structures, investigate the properties of magnetic materials in the rarely studied range of nanometer size, and exploit their extraordinary characteristics in medicine and nanoelectronics. This study consists of two parts: (i) Fabrication and study of record small magnetic tunnel junctions (ii) Introduction of a novel method for detection of magnetoelectric nanoparticles (MENs) in the tissue.

A key challenge in further scaling of CMOS devices is being able to perform non-volatile logic with near zero power consumption. Sub-10-nm nanomagnetic spin transfer torque (STT) magnetic tunneling junctions (MTJs) have the potential for a universal memory that can address this key challenge. The main problem is to decrease the switching current density. This research studied these structures in sub-10-nm size range. In this range, spin related excitations consume considerably smaller amounts of energy as compared to the larger scale. This research concluded that as predicted a decrease in switching current superior to that of the linear scaling will happen in this size range.

Magneto-electric nanoparticles (MENs) can be used to directly couple intrinsic electricfield-driven processes with external magnetic fields for controlling neural activity deep in the brain. These particles have been proven to be capable of inducing deep brain 
stimulation non-invasively. Furthermore, these magneto-electric nano-particles can be used for targeted drug delivery and are contenders to replace conventional chemotherapy. The circulatory system can deliver a drug to almost every cell in the body; however, delivering the drug specifically into the tumor cell and then releasing it on demand remains a formidable task. Nanomedicine can accomplish this, but ensuring that the drug is released at an appropriate rate once at the target site is an important task. In order to have a complete understanding of the behavior of these MENs when injected into the body, a comprehensive bio-distribution study was performed. This study introduced a novel spectroscopy method for tracing the nanoparticles in the bloodstream. This study investigated the post injection distribution of the MENs in vital organs throughout a period of two months. 


\section{TABLE OF CONTENTS}

CHAPTER

PAGE

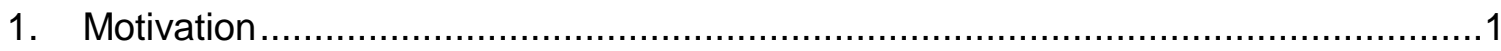

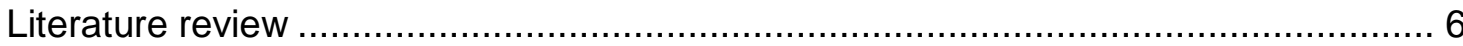

1. Magneto electric nanoparticles (MENs) in medicine ....................................... 6

Deep brain stimulation using MENs ................................................................ 6

Targeted anticancer drug delivery and release with MENs................................... 8

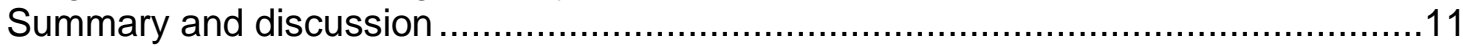

2. Fabrication of magnetic nanostructures using a focused ion beam (FIB) ...............12

Sub-10-nm magnetic tunneling junction point contacts ........................................16

Novel techniques for fabrication of sub-10-nm MTJ point contacts [61] ....................18

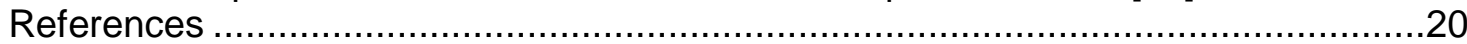

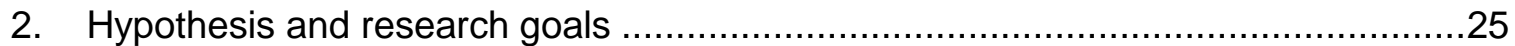

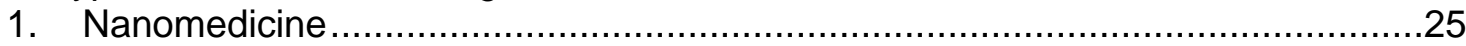

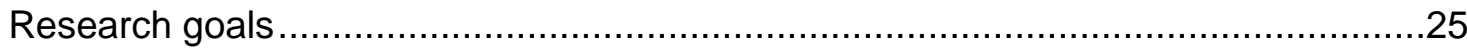

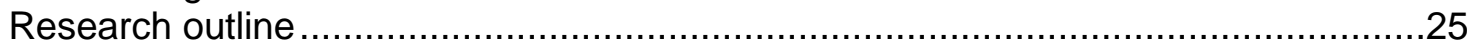

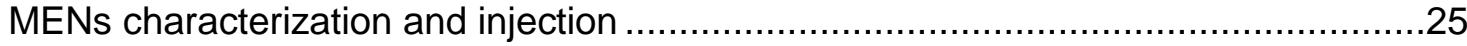

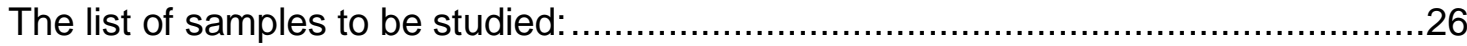

Euthanization and sample preparation for SEM imaging ........................................27

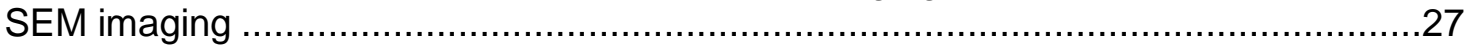

Image analysis, standardization, and data analysis of the results ..........................27

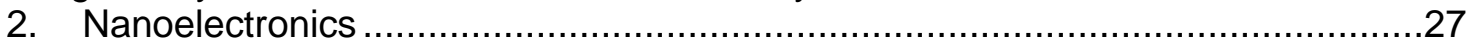

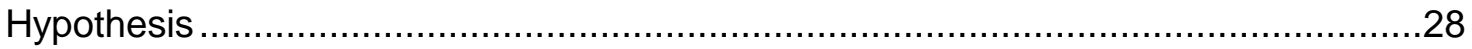

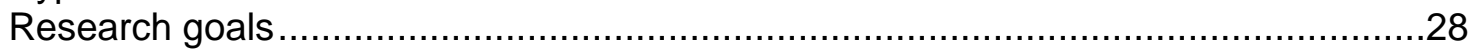

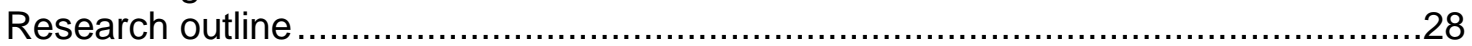

MTJ fabrication in the cleanroom ..............................................................28

Quality control testing of the fabricated junctions ……….................................29

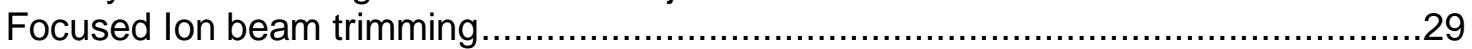

Characterization and magnetic measurement of the MTJs..................................29

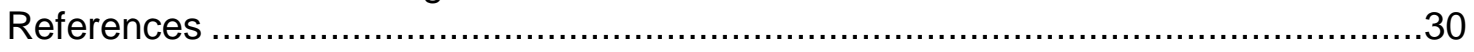

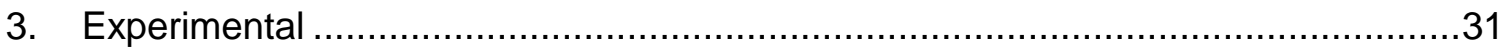

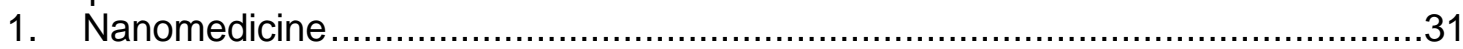

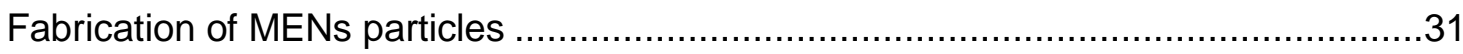

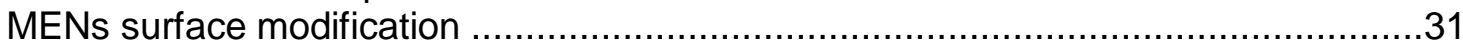

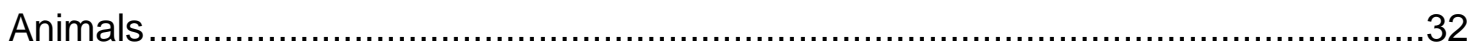

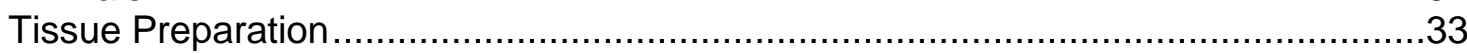

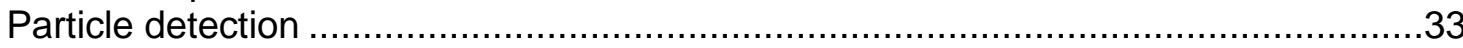

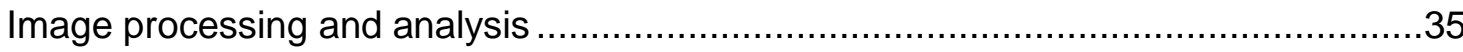

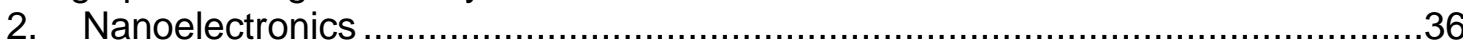

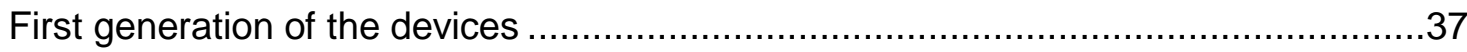

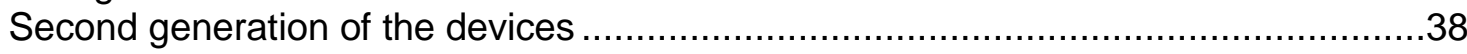

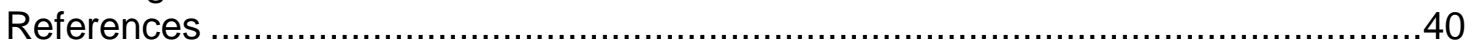

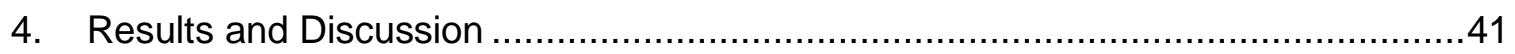

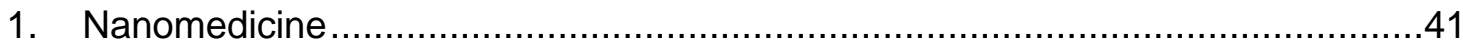

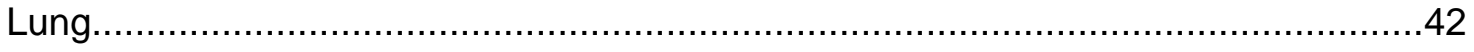




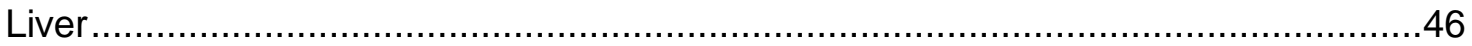

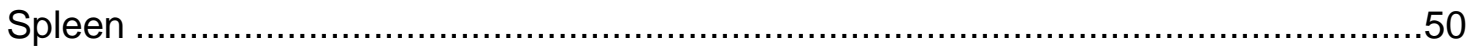

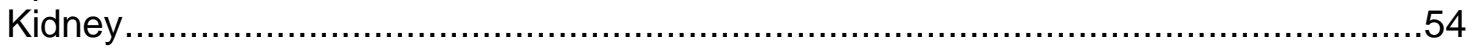

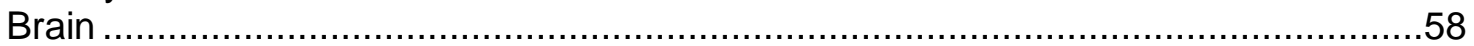

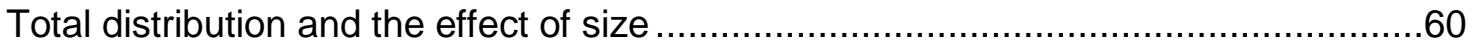

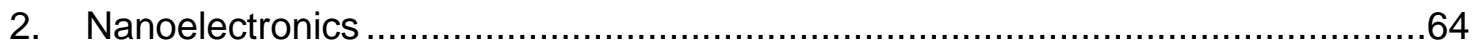

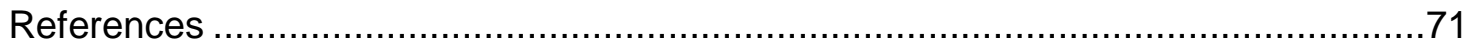

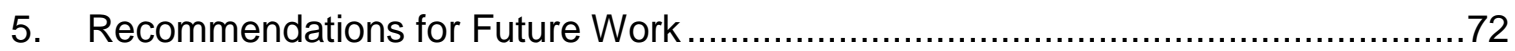

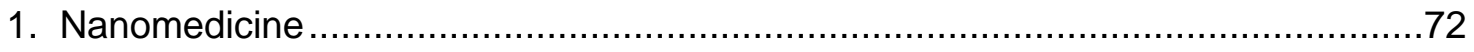

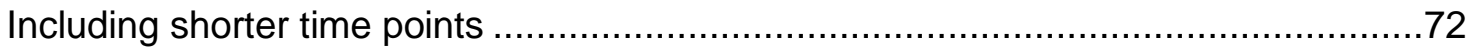

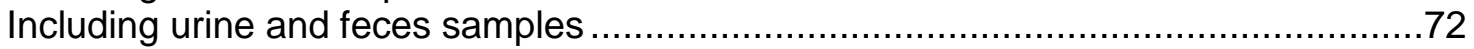

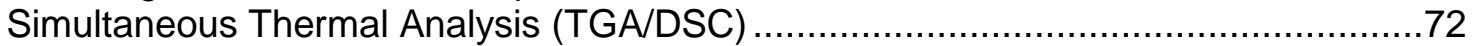

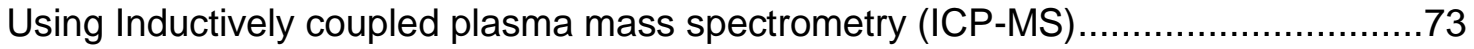

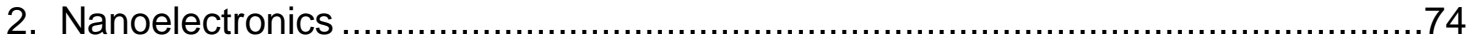

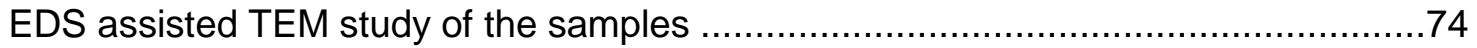

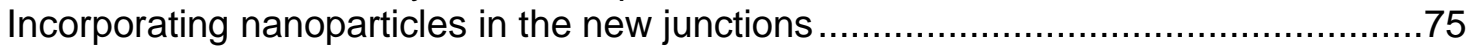

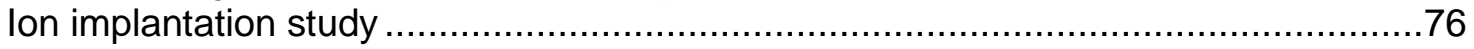

Calibration of neon and helium sources and using glass wafers instead of silicon .....76

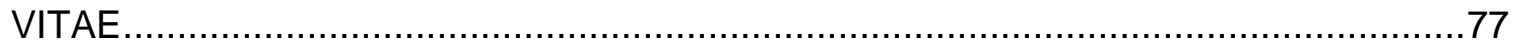




\section{LIST OF FIGURES}

FIGURE. PAGE

Figure 1.1. Schematic diagram of the sputtering process and ion-solid interactions ....... 2

Figure 1.2. Global technological installed capacity to store information [1] .................... 3

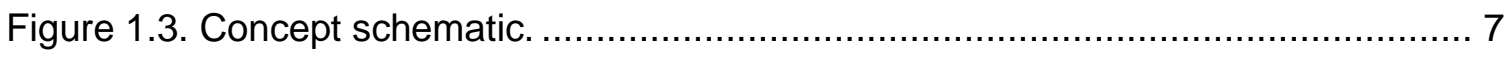

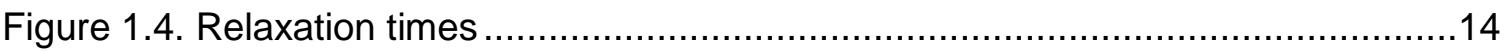

Figure 1.5. Size dependence of the switching current density ……...........................15

Figure 1.6. schematic of the junction fabricated on the probe tip [60] .........................16

Figure 1.7. M-H hysteresis loop ....................................................................17

Figure 1.8. Diamond Precision Lapping [61] …………..................................18

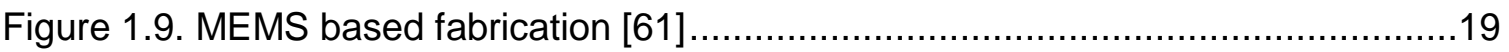

Figure 3.1. TEM image of the MENs on the left and the EDS data gathered from SEM

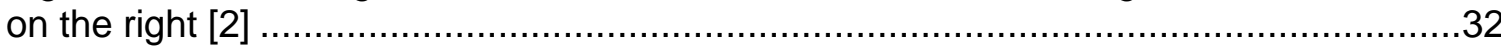

Figure 3.2. Secondary image vs backscattered image ...............................................34

Figure 3.3. Backscattered image \& mapping EDS of an area in the lung 1 month post

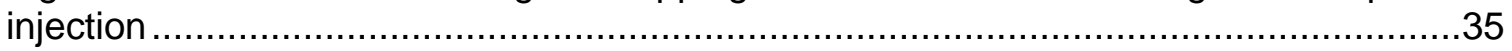

Figure 3.4. Different stages of image analysis ………………...............................36

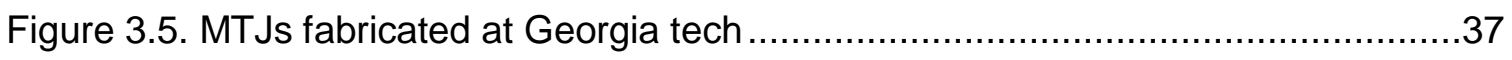

Figure 3.6. Schematic illustration ............................................................................

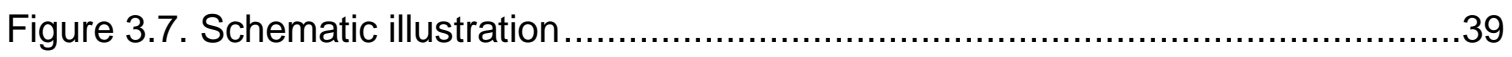

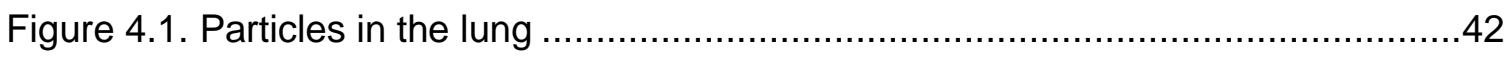

Figure 4.2. Mapping EDS of $600 \mathrm{~nm}$ MENs two months post injection in the lung ..........42

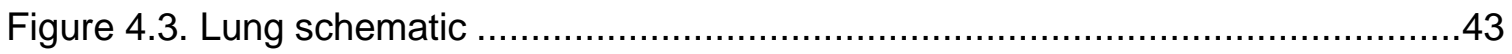

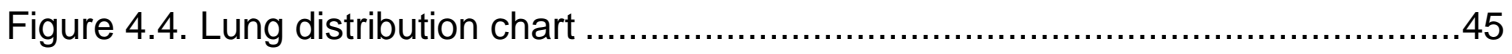

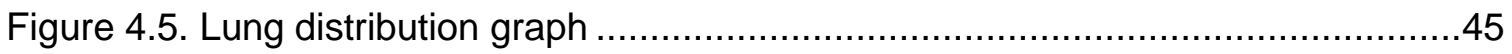

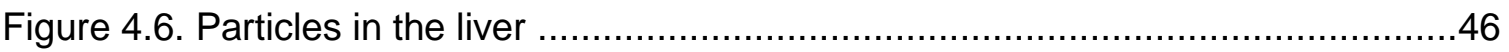


Figure 4.7. Mapping EDS of 100nm MENs a month post injection in the liver.....

Figure 4.8. Liver schematic.

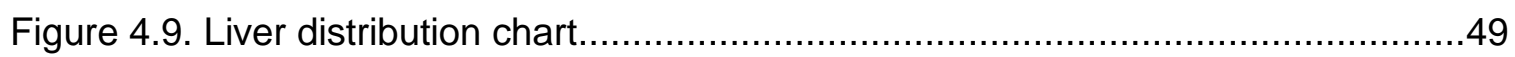

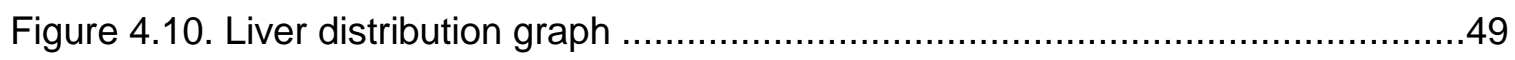

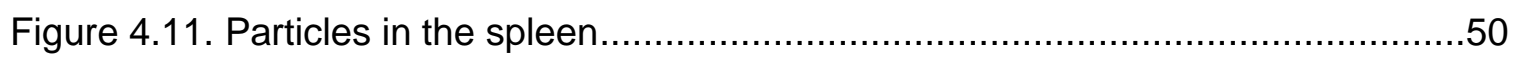

Figure 4.12. Mapping EDS of 100nm MENs a month post injection in the spleen ..........50

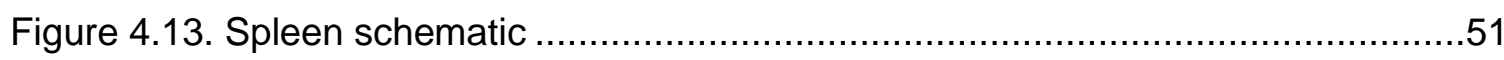

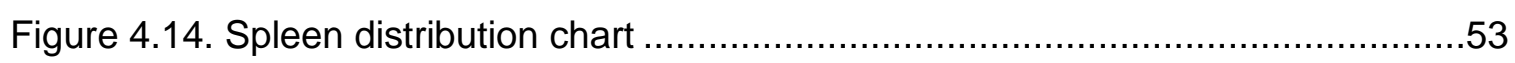

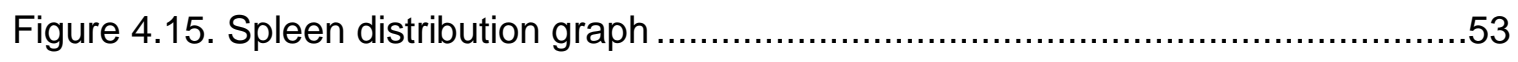

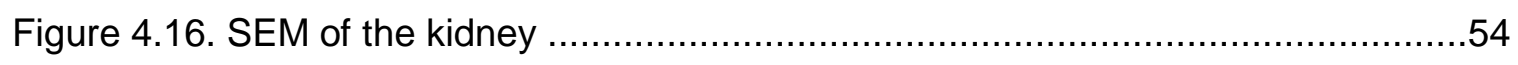

Figure 4.17. Mapping EDS of 100nm MENs a week post injection in the kidney...........54

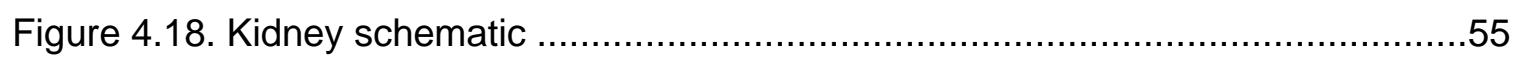

Figure 4.19. Relative anatomic location of various cell types in the kidney] ..................56

Figure 4.20. Schematic picture of the glomerular capillary ....................................56

Figure 4.21. SEM of particles in the glomerular capillary ......................................58

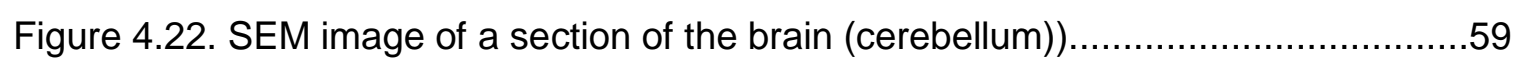

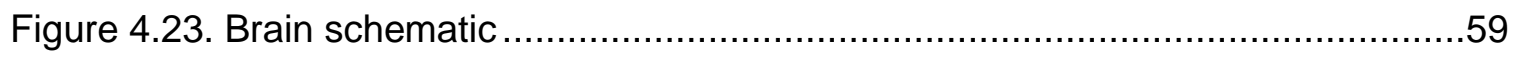

Figure 4.24. Bio-distribution of 10nm particles over time .....................................60

Figure 4.25. Bio-distribution of $30 \mathrm{~nm}$ particles over time ...................................61

Figure 4.26. Bio-distribution of $100 \mathrm{~nm}$ particles over time...................................61

Figure 4.27. Bio-distribution of $600 \mathrm{~nm}$ particles over time....................................62

Figure 4.28. Biodistribution and clearance of nanoparticles.................................63

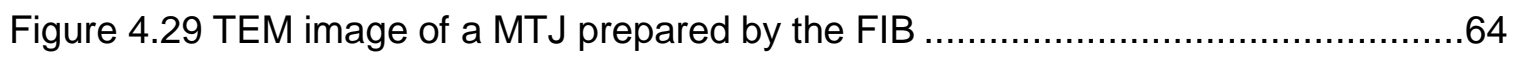

Figure 4.30. A high magnification TEM image of a MTJ showing different layers of the junction. 
Figure 4.31. M-H loop of the fabricated pristine film on the left and TMR measurement on the right

Figure 4.32. SEM image of a first generation MTJ in different steps of the milling ........66

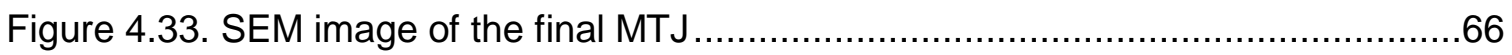

Figure 4.34. SEM image of a second generation MTJ in different steps of the milling

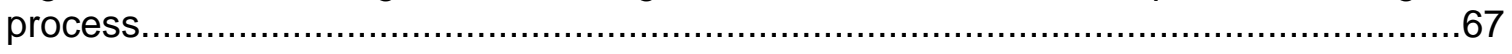

Figure 4.35. He ion image the MTJ (red box) .............................................68

Figure 4.36. The junction before any fabrication work .....................................69

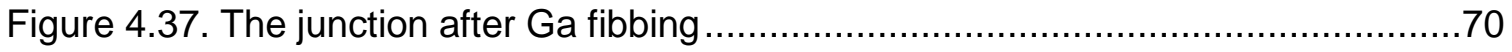

Figure 4.38. The junction after Ne patterning ............................................... 70

Figure 5.1. Approximate detection capabilities of the ICP-MS method .......................74

Figure 5.2. TEM image of a CoFe2O4 nanoparticle ............................................75 


\section{Motivation}

This research aims to investigate the properties of magnetic materials in the rarely studied range of nanometer size and exploit their characteristics in this range in medicine and nanoelectronics. This study takes advantage of a unique opportunity that has been provided by employing a focused ion beam in order to fabricate, trim, analyze and characterize the magnetic materials in both nanoelectronics and medicine. The most common dual beam focused ion beam systems combine an ion column and an electron column (i.e., a scanning electron microscope (SEM)) and possess advanced capabilities. The electron beam can be used for nondestructive imaging and characterization without sputtering the sample surface. This paves the path for very innovative ion beam millings and characterization. Additionally, three-dimensional information can be obtained by integrating the electron and ion beam operations. This procedure involves sputtering the sample in increments and imaging the sample using the SEM after each session.

A backscattered electron detector on the system can yield compositional contrast that facilitates locating and tracing the materials based on their atomic weight. In addition, an energy dispersive spectrometry (EDS) detector enables acquiring additional elemental analysis. In some cases, a combination of ion gun and SEM is being used for site-specific specimen preparation and elemental analysis. The quality of the millings, cuts or depositions depends crucially on how the impinging ion interacts with the target. Hence, having an understanding of the basics of ion beam-solid interactions may improve the ability to achieve optimum results using an FIB system greatly. The ability to mill, image, and deposit material using a focused ion beam (FIB) instrument plays a key role in the progress of this research in all the areas that are going to be discussed. For these reasons, 
a brief introduction to the nature of interaction between the ion beam and solid will be provided in the next chapter, then how these interactions are implemented in a FIB will be discussed. A description of how magnetic properties in the rarely studied range of nanometer size can be implemented in order to improve nano-electronic devices will then be followed by a discussion of the use of nanomagnetics in medicine.

Ion-Solid interactions in a focused ion beam

Figure 1.1 is a schematic that illustrates some of the ion beam-material interactions that can happen when a solid is bombarded with ions. Milling occurs due to the physical sputtering of the target.

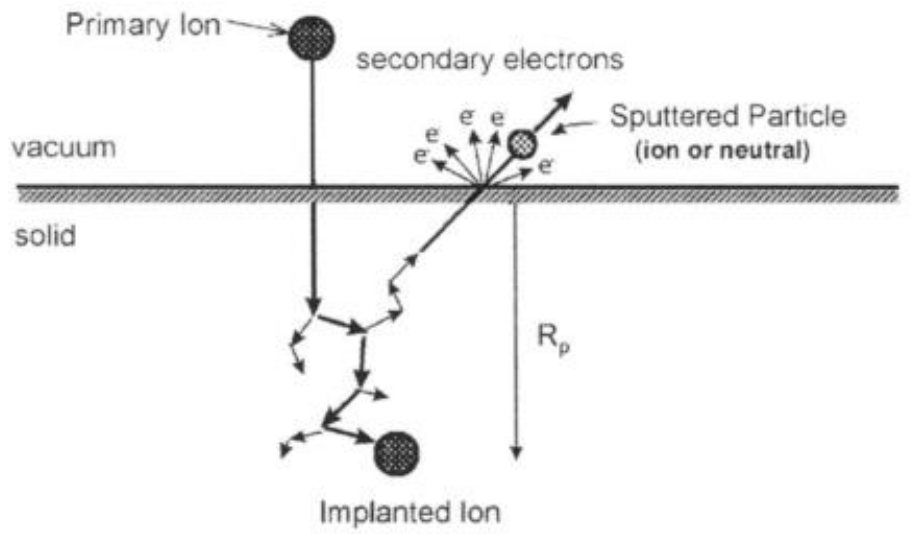

Figure 1.1. Schematic diagram of the sputtering process and ion-solid interactions

In order to understand the sputtering process, the interactions between the ion beam and the target should be considered. Sputtering takes place as a consequence of a series of elastic collisions in which momentum is transferred from the incident ions to the target atoms within a region which is called cascade region. An atom on the surface of the target may be sputtered if it receives a kinetic energy that exceeds its surface binding energy (SBE). A portion of these ejected atoms might be ionized. Because of ion bombardment, 
inelastic interactions can also happen. These interactions produce phonons, plasmons in metals, and secondary electrons (SE). A standard FIB employs secondary electrons in order to produce an image.

Nanomagnetics in Nanoelectronics

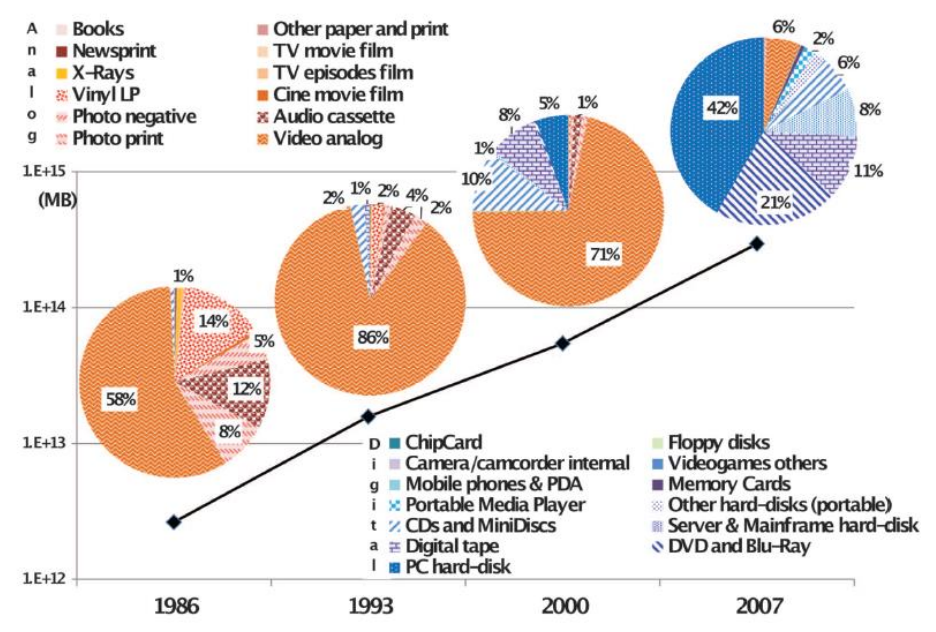

Figure 1.2. Global technological installed capacity to store information [1]

Figure 1.2 demonstrates how the storage has turned from analogue formats (orange color on the left) to digital (blue on the right) over the last decade as the information storage capacity increases. However, there are a couple of issues to be addressed in order to further scale up the storage capacity and processing speeds and scale down the size [1]. One of the main issues for further scaling of CMOS devices with non-volatile logic is the static power consumption. Spin transfer torque (STT) magnetic tunneling junction (MTJ) possesses the density advantage of dynamic random access memory (DRAM), processing speed of static random access memory (SRAM), and non-volatility of flash memory all together [2-4]. Furthermore, near zero power consumption in STT-MTJs has the potential to address the consumption problem [5]. However, the current that is required to switch the spin orientation is relatively high. This high switching current needs to be 
reduced before logic application of the STT-MTJs can be realized. There are conventional techniques for reducing the switching current, but these techniques add more complexity to the system and increase the power consumption [6]. Another possible solution can be decreasing the device size. Nevertheless, if the current density reduction abides by linear scaling, even at dimensions smaller than $10 \mathrm{~nm}$ the switching is still not energy efficient. The purpose of this study is to confirm and utilize the fact that at the sub $10 \mathrm{~nm}$ region, the switching current reduction is not occurring according to a linear scaling. The function of the thermal reservoir is to absorb the excitation energy. In the sub $10 \mathrm{~nm}$ region, the thermal reservoir becomes considerably small. Hence, it is unable to absorb the energy that was created due to the magnetic dynamics [7]. At this intermediate size range, the material can be described as a cluster. In this cluster, the excitation energy exchange is not occurring as effectively as it is in a crystal. This will result in a rapid magnetization damping drop, superior to that of a linear scaling decrease. Atomistic scale simulations confirm that the magnetization drop occurs. One of the most effective tools, which can be utilized to scale down the electronic devices are focused ion beams. These tools can be effectively used to reduce the size of the devices. As a part of this research, different ion beams with different beam currents are employed to mill down a magnetic tunneling junction. The aim is to fabricate the smallest sizes which were reported in order for the magnetic properties to be studied in these rarely studied ranges.

\section{Nanomagnetics in Medicine}

Magneto-electric nanoparticles (MENs) are nanoparticles with a coreshell nanostructure composed of a core of $\mathrm{CoFe} 2 \mathrm{O} 4$ around 7-10 nm in diameter and a shell $\mathrm{BaTiO} 3$ with a diameter, which is around $30 \mathrm{~nm}$. They are synthesized through a standard hydrothermal methods. These MENs can be used in order to directly couple intrinsic electric-field-driven 
processes with external magnetic fields for controlling neural activity deep in the brain. These particles have been proven to be able to induce deep brain stimulation noninvasively. Additioanlly, these magneto-electric nano-particles can be used for targeted drug delivery and are strong contenders for alternatives to conventional chemotherapy. The circulatory system can deliver a drug to almost every cell in the body; however, delivering the drug specifically into the tumor cell and then releasing it on demand remains a formidable challenge. Ensuring that the drug is not prematurely released in the plasma or interstitial space but is released at an appropriate rate once at the target site is an important task. In order to have a complete understanding of the behavior of these MENs when injected into the body, a comprehensive bio-distribution study was required. This bio-distribution study investigated the post injection distribution of the MENs in different organs throughout a period of two months in a mouse model. A JEOL 4500 dual beam SEM, which was equipped with a back scattered and energy dispersive spectroscopy detector was employed. These detectors were used in order to locate, confirm, and map the particles in each organ. Each mouse was injected with a single dose of nanoparticles in one of four sizes $(10 \mathrm{~nm}, 30 \mathrm{~nm}, 100 \mathrm{~nm}$, or $600 \mathrm{~nm})$ and was terminated after a predetermined time point (24 hours, 1 week, 1 month, and 2 months). Different organs were harvested and sectioned for scanning electron microscopy (SEM) studies. This study aims to gain a comprehensive understanding of what portion of the particles go to which specific organ after entering the blood stream, if and how they cluster, how long they stay in each site, and how long it takes for the particle clusters to grow smaller in size and quantity. 


\section{Literature review}

\section{Magneto electric nanoparticles (MENs) in medicine}

In order to illustrate the significance of studying the bio-distribution of nanoparticles after entering the blood stream and over time, a few applications for MENs in different branches of medicine will be discussed in this section.

\section{Deep brain stimulation using MENs}

A neural network can be described as a complex bio-electric circuit which consists of many neurons connected through chemical and electrical synapses formed between axons and dendrites [8-10]. The signaling in the network is often driven by electric fields and is based on a highly collective system of electric charges, neurotransmitters, and action potentials. The ability of remotely stimulating specific neuronal excitations deep in the brain with the intent to trigger selective regions of the network remains an important unanswered question in neural engineering [11]. Additionally, being able to control the central nervous system (CNS) at micro- or even nano-scale could provide exceptional control over specific functions and make highly personalized "pin-point" treatment of neurodegenerative diseases such as Parkinson's Disease (PD), Essential Tremor (ET), Epilepsy, and others possible $[12,13]$.

The in-vivo study on imprinting control region mice shows MENs can be implemented in order to couple intrinsic electric field-driven processes with external magnetic fields and may be used to control neural activity deep in the brain. [14] 


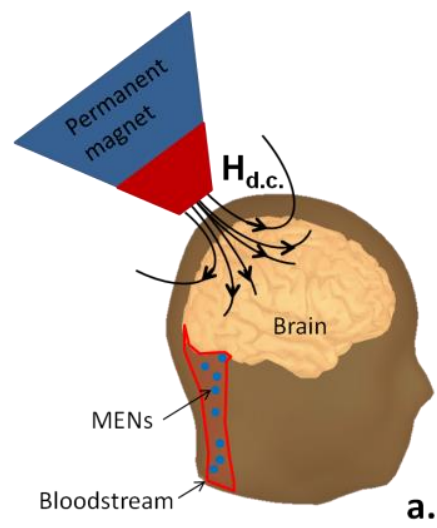

a.

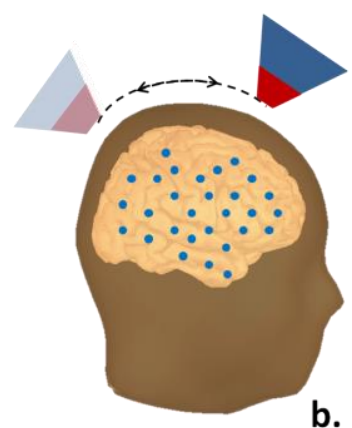

b.

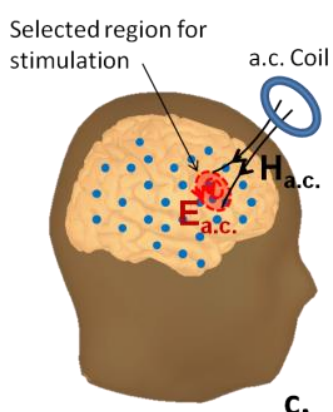

C.

Figure 1.3. Concept schematic

Illustration of the novel concept of using MENs for "mapping" the brain for non-invasive electric field stimulation of selected regions deep in the brain. (a) MENs are dragged into the brain across the BBB via application of a d.c. magnetic field gradient. (b) When in the brain, MENs are being distributed over the entire brain or in selected regions via application of spatially varying d.c. magnetic field gradients. The existence of MENs forms a "new brain microenvironment", in which the intrinsic electric signals created because of the neural activity are intensely coupled at the sub-neuronal level to the external magnetic fields generated by remote source. (c) This coupling can be used for non-invasive high-efficacy stimulation of selected regions deep in the brain via application of a relatively low $(\sim 100 \mathrm{Oe})$ near-d.c. $(\sim<1000 \mathrm{~Hz})$ magnetic field.

In this study, approximately $10 \mu \mathrm{g}$ of $\mathrm{CoFe} 2 \mathrm{O} 4-\mathrm{BaTiO} 330-\mathrm{nm}$ nanoparticles were injected into the blood stream of a mouse through the tail vein and were forced to cross the blood-brain barrier (BBB) via a d.c. field gradient. The toxicity was eliminated through coating the nanoparticles with a layer of glycerol monooleate of a thickness of $2 \mathrm{~nm}$. After the nanoparticles reached the brain, there were over 40 nanoparticles per each neuron. This was confirmed through SEM imaging of brain slices as well. A surgically attached two-channel EEG headmount was employed in order to show that the administrated MENs could modulate the electric waveforms deep in the brain via application of a magnetic field with a frequency ranging from 0 to $20 \mathrm{~Hz}$. To further emphasize the significance of this study, it is worth noting that introduction of MENs into the brain could pave the path for "writing" and "reading back" an electric field map of the brain at a sub-neuronal level. A 3D electric field mapping via these particles can be used for both remote stimulation of specific 
functions and "reading back" the electric field information with a sub-neuronal precision, assuming there is an efficient magnetic imaging technique available. Additionally, it should be noted that the MENs' parameters that were mentioned above are not limited to the CoFe2O4-BaTiO3 composition and the $30 \mathrm{~nm}$ diameter, as used in the aforementioned study. It is possible to further tailor and improve upon the application by using different materials, e.g. biodegradable compositions, and even smaller size nanoparticles [14]. It is commonly assumed that approximately $10 \%$ of IV-administrated nanoparticles transfer into the brain [15]. However, in this particular case, this estimate might be conservative taking into account that a "magnetic field gradient pull" was used to force the nanoparticles to cross BBB. A more comprehensive in-vivo study had to be conducted to fully comprehend the detailed metabolism of the nanoparticles, including understanding exactly how much of the particles reach the brain and how they are distributed throughout the other organs. It was also important to learn how long the particles take to reach their destinations and how long they remain there. A set of experiments using an energydispersive spectroscopy (EDS) based analysis of the nanoparticle distribution in different organs were designed to address this need. This detailed study is going to be discussed in the following chapters. This study is crucial to the next stages of this research and also other bodies of research which involve using these nanoparticles as agents, drug carriers, etc. These studies require biodistribution analysis of MENs of different compositions, sizes, and shapes observed in different organs and in different time intervals after the administration.

\section{Targeted anticancer drug delivery and release with MENs}

A significant challenge in cancer treatment in general is developing a controlled targeted delivery and release of cancer medications with the aim of eradication of tumor cells while leaving the normal cells unharmed. The circulatory system is capable of delivering a drug 
to almost every cell in the body. However, carrying the drug, specifically into the tumor cell past its membrane, and then releasing the drug into the tumor cells on demand without affecting the normal cells remains a challenge [16-18].

Recent research is trying to address this important issue by employing nanoparticles as drug carriers [19-21].Nanoparticles possess unique properties due to the following reasons: (i) They have unique sizes, which range from tens to over one hundred nanometers. This enables tailoring drug delivery into different organs. (ii) They have a wide shape variation. This includes spheres, rods, and platelets. This property helps to steer the drug-loaded nanoparticles towards more specific targets. (iii) It is possible to perform comprehensive surface functionalization on these particles. These attributes are important because there is need to meet a wide range of applications, which are required for conjugation with specific biomolecules, and in order to surpass numerous biological barriers. These processes can occur with or without exploiting the immune system. Last but not least, nanoparticle drug delivery (NDD) shows potential for addressing the fundamental issue of multidrug resistance (MDR) in cancer therapies. NDD systems take advantage of multiple material and environmental compositions including metal and polymer nanostructures, thermally responsive polymers, electromagnetically (in UV, Visible-Wavelength, and IR ranges) or acoustically activated materials, liposomes, electrochemical processes, and magnetic fields [22]. There is another advantage, which makes magnetic nanoparticle systems exceptional: they can be remotely navigated to an intended site through application of an external magnetic field gradient [23]. Accumulations of systematically administered nanoparticles have been observed in a number of tumors due to the enhanced permeability and retention (EPR) effect. In short, EPR is established dye to the high leakiness of tumor blood vessels and due to the fact that tumors lack an adequate lymphatic system [24-27]. For a successful vascular delivery 
of cancer drugs, particles should have a small size $(<\sim 200 \mathrm{~nm}$ but $>\sim 10 \mathrm{~nm})$ and also a neutral charge and hydrophilic coating [43].

Exceptionally small particles $(<\sim 10 \mathrm{~nm})$ can be discharged by the kidney and larger particles (> $200 \mathrm{~nm}$ ) can be discharged by the mononuclear phagocyte system (MPS). Recently, special attention has been devoted to advancing the immunotherapy-mediated active nanoscale approaches. In one case, for example, monoclonal antibodies (mAbs) were employed to identify over-expressed tumor specific biomarkers, while nanoparticles were used as a high-efficiency drug delivery system [28-33]. In spite of the great promise of the nanoparticle delivery, making sure that the drug is not prematurely released in the plasma or interstitial space remains a significant challenge. The drugs are supposed to be released at an appropriate rate once at the intended site, i.e. into the cancer cell cytoplasm [34]. To address this problem, nanoparticles have been modified to trigger the drug release by different methods. These methods include triggering drug release by externally applied temperature [35, 36], ultrasound [37, 38], intracellular $\mathrm{pH}$ [39], intracellular enzymes [40, 41], or the tumor microenvironment [42]. All these methods still have the disadvantage of inconsistent drug release when the delivery nanoparticles reach the targeted organ. In actuality, employing NDD systems to control retention and for targeted drug delivery stays an open question in cancer treatment.

A combined in-vitro and in-vivo study by our group demonstrates how MENs can be employed in order to externally capacitate controlled high-specificity targeted delivery and release on demand [43]. Additionally, possessing this amount of control paves the path for physically separating the two crucial functions of drug delivery and release via applying d.c. and a.c. magnetic fields. This amount of control can be accomplished here because, unlike standard purely magnetic nanoparticles like iron oxide nanostructures, MENs have a non-zero magnetoelectric (ME) effect. This is because of their strongly coupled 
magnetostrictive and piezoelectric properties [43]. It is well established that cellular membranes are electrically charged. Hence, MENs can interact with the cellular microenvironment through electric fields. Furthermore, due to the ME effect, MENs provide a unique opportunity to employ magnetic fields in order to externally control intrinsic electric fields which inhibit the chemical bonds between the nanoparticles and the drug which has been mounted on it. Additionally, these fields can be used to control the interaction between the drug carrier and the cellular microenvironment [44-47]. It has been shown that the electric properties of the cancer cells differ from the normal cells. These differences include membrane potential, cellular dielectric constant, or electric capacitance $[48,49]$. As an example, the membrane potential for ovarian cancer cells in interphase is around $-3 \mathrm{mV}$ whereas normal ovarian cells have a membrane potential of around -50 mV [50]. Consequently, the electric-field interaction between MENs and cancer cells is different from the interaction between MENs and normal cells. It has been shown that externally controlled MENs can be used in order to carry, deliver, and release the drugs at the intended sites. MENs carry a payload of drugs and deliver them to the intended target site. They avoid removal from the circulatory system before they reach the target, enter the cancer, and do not affect the normal cells. MENs release the drug only after the carrier nanoparticles enter the cancer cells through application of a specific sequence of external d.c. and a.c. fields on demand [43]. The proposed method for targeted drug delivery by MENs can be applied in order to cure any cancer; however, this was a case study focused on ovarian cancer $(\mathrm{OC})$, which is characteristically metastatic (Stage III) at the time of diagnosis [51, 52].

\section{Summary and discussion}

The aforementioned examples are only a few of the many applications that MENs can have. Particle imaging using MENs, deep brain stimulation, designing a brain map, 
targeted drug delivery, and all the other applications which can be designed for these particles are not going to be complete unless a comprehensive bio-distribution study is performed. Different parameters might affect the result of this study and the size of the particle is one of the important parameters that is going to influence how long the particle stays in the blood stream and how it is discharged from the body. The study covers different particle sizes ranging from $10 \mathrm{~nm}$ to $600 \mathrm{~nm}$. The data has to be collected in different times after the injection of the MENs and the analysis has to be performed on vital organs such as lung, liver, kidney, spleen, and brain. In the following sections, the design and results of such a study will be discussed.

\section{Fabrication of magnetic nanostructures using a focused ion beam (FIB)}

Conventional magnetic materials have served different purposes, from electric motors to magnetic compasses and sensors. Recently, a new family of magnetic materials are becoming more popular. This new family of materials functions based on the manipulation of spin polarized electrons in magnetic thin-film structures. The advent of magnetoresistance and the discovery of exchange coupling around two decades ago has enabled the engineering and design of a new class of thin-film magnetic materials for advanced magnetic sensors and magnetic random access memory. The advent of spinvalve magnetoresistive (GMR) sandwiches has paved the path for fabrication of magnetic sensors, which have increased the storage capacity of magnetic hard drives enormously. The discovery of magnetic tunnel junctions has enabled the design of a nonvolatile magnet random access memory, which has a density close to that of a dynamic random access memory and read write speeds close to that of a static RAM. GMR and MTJ based devices are examples of spintronics in application. In both of these, the spin-polarized currents of electrons are being manipulated by magnetic fields [53]. 
Spin transfer torque (STT) magnetic tunneling junction (MTJ) have the potential to be used as a universal memory that has a density in order of a DRAM, the speed advantage of SRAM, and is nonvolatile just like flash [54-56]. Furthermore, STT-MTJs have the promise of non-volatile logic with a static power consumption, which is near zero, and this is a fundamental issue in further scaling of CMOS devices. Nevertheless, the energy, which is required to switch the spin orientations in these devices, is comparatively high. This high energy consumption for switching the spin remains the main obstacle for this technology to become competitive. Switching current reduction methods such as heat-assisted switching and voltage-controlled anisotropy can be employed in order to reduce the required current [57]. However, these techniques add to the complexity of the structure and demand additional power consumption for the heat source and voltage bias. This study intends to explore the sub-10-nm region where, according to simulations and calculations, a switching current reduction superior to the linear area scaling is expected. In this region, the thermal reservoir, whose functionality is to absorb the energy of excitations, becomes exceedingly small and becomes unable to absorb the energy, which is a result of the collective spin dynamics [58].

According to the commonly accepted phenomenological expression by Slonczewski, the switching current density $J_{S}$ is changing in a linear fashion according to [56]:

$$
J_{s} \sim \frac{M_{s} t \alpha}{p \xi}\left(H_{k e f f}+H_{0}\right)
$$

$H_{k e f f}$ is the anisotropy field and takes into account both intrinsic and shape anisotropy. $H_{0}$ Is the net external field. $\alpha$ accounts for the damping constant ranging from 0.001 to 0.1. $\xi$ is the spin torque efficiency factor. $M_{s}$ is the saturation magnetization. $t$ is the thickness of the free layer. $p$ is the spin polarization and is related to the magnetization according to Jullier equation: 


$$
\frac{\Delta R}{R}=\frac{2 P^{2}}{\left(1-P^{2}\right)}
$$

As is common for conventionally "large" sizes of the device (> 10nm), the crystalline fields and elastic modulation of exchange interaction forms a coupling to phonons. L-S interactions take part in fast and slow spin-lattice relaxation mechanisms. Spin-lattice relaxation and thermal fluctuations from the thermal reservoir cause a destruction in the Hamiltonian behavior of the spin system and hence destroy the coherent long-lived signals. In the sub-10nm range, ab initio calculations would directly take into account all the quantum-mechanical interactions between adjacent spins [58]. In the case of the sub $10 \mathrm{~nm}$ range, the spin relaxation time is expected to rise by orders of magnitude as the size is further decreased below approximately $5 \mathrm{~nm}$.
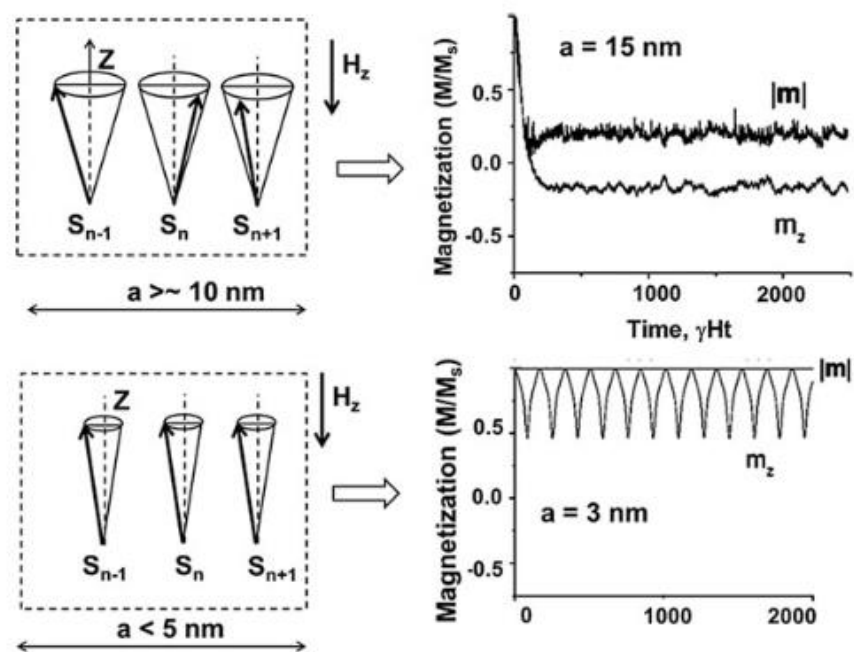

Figure 1.4. Relaxation times for sizes above and under the thresholdthe relaxation process in two regions of interest, (i) above-10-nm range (top row) and (ii) sub10-nm range (bottom row) [59]

According to one model, the effect can be explained if the surface and volume effects are separated. The expression for spin relaxation time is as follows: 


$$
\tau_{s} \sim \frac{d}{V_{f}}\left[\Delta g_{v}^{2}\left(1-\frac{N_{s}}{N_{v}}\right)+\Delta g_{s}^{2}\left(\frac{N_{s}}{N_{v}}\right)\right]^{-1}
$$

$d$ Is the nano-structure parameter. $\Delta g_{v}$ and $\Delta g_{s}$ are the $g$-factor shifts for bulk and surface electrons. $N_{v}$ and $N_{s}$ are the electron concentrations for bulk and surface. In general, the surface shift $\Delta g_{s}$ happens to be smaller than the volume shift $\Delta g_{v}$ by orders of magnitude [6]. Hence, as the size is decreased to where the surface effects are dominant, the spin relaxation times increase by orders of magnitude.

This implies that above a certain threshold size, the device acts like a crystal and according to the established dependence that is characteristic of a crystal structure. It has been confirmed that focused ion beam fabricated devices with a size above $10 \mathrm{~nm}$ exhibit a switching current density that is on a par with the other literature reported measurements. However, below the threshold size, the surface effects become more dominant. This domination of surface effects is because of collective spin excitations. As a result, the spin relaxation time can be substantially increased in this case [60].

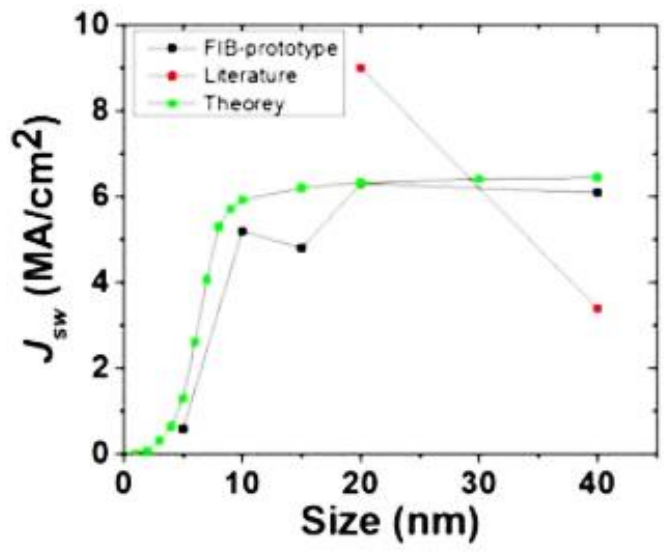

Figure 1.5. Size dependence of the switching current density Green dots show the theoretical points, red dots are the data from literature and black dots resulted from the fib prototyping [60] 
Different approaches have been proposed in order to perform the study in sub $10 \mathrm{~nm}$ range and a couple of them will be discussed. Then methods and set up of the current study will be explained in detail.

\section{Sub-10-nm magnetic tunneling junction point contacts}

In this study, in order to achieve a small MTJ cross-section, a point contact between a nanoprobe and the surface of a metal (copper) substrate was established. A scanning tunneling microscopy (STM) probe tip was tuned by focused ion beam (FIB) in order to make the tip as sharp as possible. Then the probe was sputtered with a commonly used MTJ multilayer composition, which is $\mathrm{Ta}(5 \mathrm{~nm}) / \mathrm{CoFeB}(1 \mathrm{~nm}) / \mathrm{MgO}(0.9 \mathrm{~nm}) / \mathrm{CoFeB}(1$ $\mathrm{nm}) / \mathrm{Ta}(5 \mathrm{~nm})$, as shown:

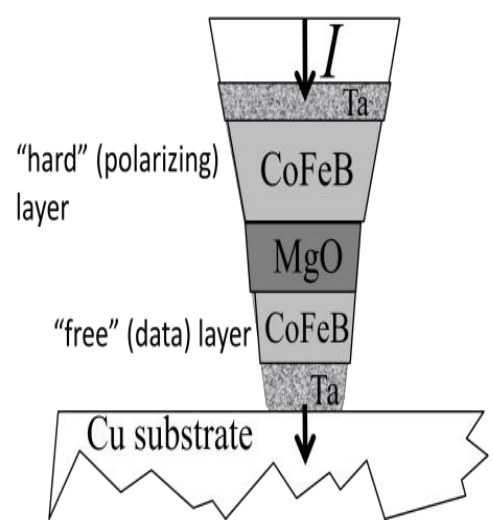

Figure 1.6. schematic of the junction fabricated on the probe tip [60]

These layers were deposited under a 10-mTorr argon with a sample rotation in order to make sure the surface of the sample is being uniformly and completely covered. In this case, the last ferromagnetic layer which was sputtered on the tip of the probe works as the data storage "free" layer. This assumption was confirmed by Landau- Lifshitz-Gilbert 
(LLG) model-based micromagnetic simulation results. The coercivity of the "free" layer is expected to be smaller than 50 Oe while the coercivity of the "fixed" layer is around 100 Oe. This composition is chosen in order to provide the optimum combination of the properties, which are crucial for the device. These properties include high spin torque efficiency, high magnetoresistance and ultra-high interface-induced perpendicular anisotropy energy. High spin torque efficiency is needed to strengthen the STT effect. High magnetoresistance improves the signal to noise ratio (SNR) and ultra-high interfaceinduced perpendicular anisotropy makes sure that the desired thermal stability is maintained as the size is reduced to sub $10 \mathrm{~nm}$. In this case the stability condition is:

$$
\frac{K_{u} V}{K_{B} T}>20
$$

Here $K_{u}$ is the anisotropy energy density. $V$ is the free layer volume. $K_{B}$ is the Boltzmann constant, and $T$ is the ambient temperature. Taking advantage of a focused MagnetoOptical Kerr Effect (f-MOKE) magnetometer, the interface-induced perpendicular anisotropy field was measured at $\sim 5 \mathrm{kOe}$ and the out-of-plane coercivity field measured at $\sim 75$ Oe for this composition in the free layer is shown in figure 1.6:

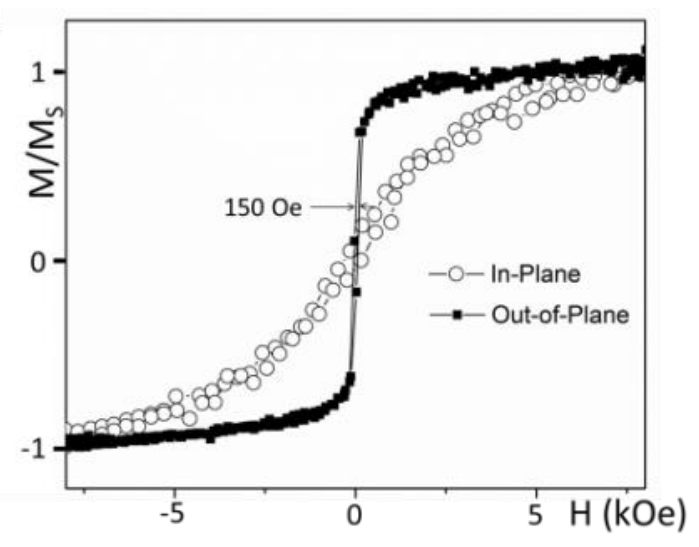

Figure 1.7. M-H hysteresis loop in-plane and out of-plane $\mathrm{M}-\mathrm{H}$ hysteresis loops measured by f-MOKE on the free layer [60] 
The contribution of the shape of the aforementioned nano-junction made of this material is going to increase the net anisotropy even further. Additionally, a final layer of deposited Ta not only improves the perpendicular anisotropy but also protects the nanoscale junction against oxidation. Also, this coating guarantees that an adequate contact with the interfacing metal surface exists. A session of FIB milling ensured the removal of the potential Ta film coating on the side regions. In order to take into account the contribution of the metal substrate, graphite was used as an alternative to copper and the results showed no noticeable change. The measured switching current was around 100nm (which is approximately a current density of the order of $\left.10^{5} \mathrm{~A} / \mathrm{cm}^{2}\right)$. This switching current is at least an order of magnitude smaller than the value that is expected from the linear scaling of the junction cross-section area of the smallest reported current density.

\section{Novel techniques for fabrication of sub-10-nm MTJ point contacts}

The preliminary results were encouraging enough to advance with more robust and scalable fabrication methods. This section briefly describes some alternative approaches: (i) Diamond Precision Lapping that allows for removing the region of interest using a mechanical lapping and polishing process. This removes the requirement of using FIB but this method is not as precise and the resolution is usually limited. In addition, contamination due to the polishing media can cause complications [61].

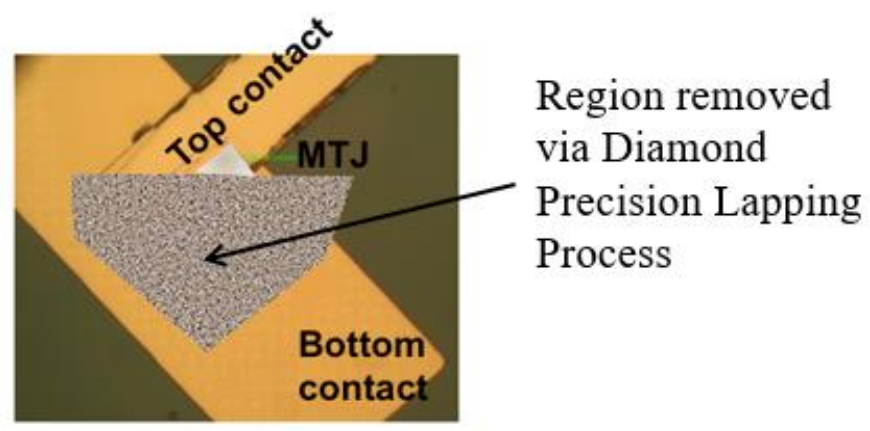

Figure 1.8. Diamond Precision Lapping [61] 
(ii) Another approach that has the advantage of being mass producible is MEMS based fabrication that takes advantage of lithography and etching techniques in order to make a small trench in which the junction will then be deposited[61].

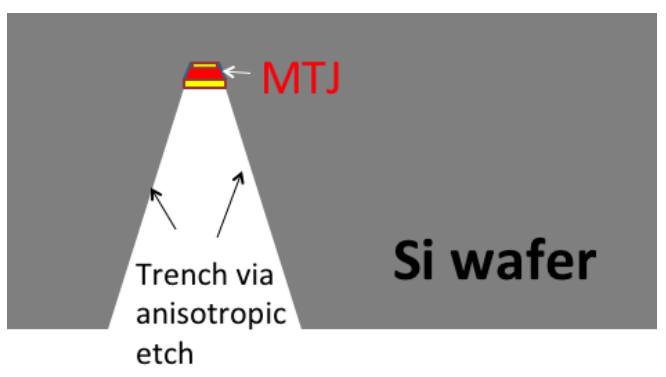

Figure 1.9. MEMS based fabrication [61]

A couple of other methods show promise for making sub $10 \mathrm{~nm}$ mangnetic tunneling junctions. Of these, using magneto-electric nanoparticles and using E-beam lithography are worthy of mentioning [61]. 


\section{References:}

1. Hilbert, Martin, and Priscila López. "The world's technological capacity to store, communicate, and compute information." science 332.6025 (2011): 60-65.

2. Tsoi, M., et al. "Excitation of a magnetic multilayer by an electric current."Physical Review Letters 80.19 (1998): 4281.

3. Slonczewski, J. C. "Currents and torques in metallic magnetic multilayers."Journal of Magnetism and Magnetic Materials 247.3 (2002): 324-338.

4. Berger, L. "Emission of spin waves by a magnetic multilayer traversed by a current." Physical Review B 54.13 (1996): 9353.

5. Prejbeanu, I. L., et al. "Thermally assisted switching in exchange-biased storage layer magnetic tunnel junctions." Magnetics, IEEE Transactions on40.4 (2004): 2625-2627.

6. Gajek, M., et al. "Spin torque switching of $20 \mathrm{~nm}$ magnetic tunnel junctions with perpendicular anisotropy." Applied Physics Letters 100.13 (2012): 132408.

7. Safonov, Vladimir L. Nonequilibrium magnons: Theory, experiment and applications. John Wiley \& Sons, 2012.

8. Marblestone $\mathrm{AH}$ et al. Physical principles for scalable neural recording. Front. Comput. Neurosci. 7 (2013).

9. Hopfield, John J. "Neurons with graded response have collective computational properties like those of two-state neurons." Proceedings of the national academy of sciences 81.10 (1984): 3088-3092.

10. De Kramer, J. J. "The electrical circuitry of an olfactory sensillum in Antheraea polyphemus." The Journal of neuroscience 5.9 (1985): 2484-2493.

11. Srikanth, Maya, and John A. Kessler. "Nanotechnology-novel therapeutics for CNS disorders." Nature Reviews Neurology 8.6 (2012): 307-318.

12. Yue, Kun, et al. "Magneto-electric nano-particles for non-invasive brain stimulation." PloS one 7.9 (2012): e44040.

13. Brambilla, Davide, et al. "Nanotechnologies for Alzheimer's disease: diagnosis, therapy, and safety issues." Nanomedicine: Nanotechnology, Biology and Medicine 7.5 (2011): 521-540.

14. Guduru, Rakesh, et al. "Magnetoelectric'spin'on stimulating the brain."Nanomedicine 10.13 (2015): 2051-2061. 
15. Calvo, Pilar, et al. "Long-circulating PEGylated polycyanoacrylate nanoparticles as new drug carrier for brain delivery." Pharmaceutical research 18.8 (2001): 1157-1166.

16. Kantoff, Philip W., et al. "Sipuleucel-T immunotherapy for castration-resistant prostate cancer." New England Journal of Medicine 363.5 (2010): 411-422.

17. Lee, Daniel W., et al. "The future is now: chimeric antigen receptors as new targeted therapies for childhood cancer." Clinical Cancer Research 18.10 (2012): 2780-2790.

18. Vasir, Jaspreet K., and Vinod Labhasetwar. "Targeted drug delivery in cancer therapy." Technology in cancer research \& treatment 4.4 (2005): 363-374.

19. Wang, Andrew Z., Robert Langer, and Omid C. Farokhzad. "Nanoparticle delivery of cancer drugs." Annual review of medicine 63 (2012): 185-198.

20. Brigger, Irène, Catherine Dubernet, and Patrick Couvreur. "Nanoparticles in cancer therapy and diagnosis." Advanced drug delivery reviews 64 (2012): 24-36.

21. Pridgen, Eric M., Robert Langer, and Omid C. Farokhzad. "Biodegradable, polymeric nanoparticle delivery systems for cancer therapy." (2007): 669-680.

22. Arruebo, Manuel, et al. "Magnetic nanoparticles for drug delivery." Nano today 2.3 (2007): 22-

23. Hoare, Todd, et al. "A magnetically triggered composite membrane for on-demand drug delivery." Nano letters 9.10 (2009): 3651-3657.

24. Brasseur, Francis, et al. "Actinomycin D adsorbed on polymethylcyanoacrylate nanoparticles: increased efficiency against an experimental tumor." European Journal of Cancer (1965) 16.11 (1980): 1441-1445.

25. Maeda, Hiroshi. "The enhanced permeability and retention (EPR) effect in tumor vasculature: the key role of tumor-selective macromolecular drug targeting." Advances in enzyme regulation 41.1 (2001): 189-207.

26. Prabhakar, Uma, et al. "Challenges and key considerations of the enhanced permeability and retention effect for nanomedicine drug delivery in oncology."Cancer research 73.8 (2013): 2412-2417.

27 Oldham, Robert K., and Robert O. Dillman. "Monoclonal antibodies in cancer therapy: 25 years of progress." Journal of Clinical Oncology 26.11 (2008): 1774-1777.

28. Tai, Wanyi, Rubi Mahato, and Kun Cheng. "The role of HER2 in cancer therapy and targeted drug delivery." Journal of Controlled Release 146.3 (2010): 264-275.

29. Li, Xin, Qinghe Zhao, and Liyan Qiu. "Smart ligand: aptamer-mediated targeted delivery of chemotherapeutic drugs and siRNA for cancer therapy."Journal of Controlled Release 171.2 (2013): 152-162. 
30. Wei, Xiaoli, et al. "Brain tumor-targeted drug delivery strategies." Acta Pharmaceutica Sinica B 4.3 (2014): 193-201.

31. Firer, Michael A., and Gary Gellerman. "Targeted drug delivery for cancer therapy: the other side of antibodies." Journal of hematology \& oncology 5.1 (2012): 1-16.

32. Barua, Sutapa, et al. "Particle shape enhances specificity of antibody-displaying nanoparticles." Proceedings of the National Academy of Sciences110.9 (2013): 32703275.

33. Danhier, Fabienne, Olivier Feron, and Véronique Préat. "To exploit the tumor microenvironment: passive and active tumor targeting of nanocarriers for anti-cancer drug delivery." Journal of Controlled Release 148.2 (2010): 135-146.

34. Zhang, Wujie, et al. "Synthesis and characterization of thermally responsive pluronic F127- chitosan nanocapsules for controlled release and intracellular delivery of small molecules." ACS nano 4.11 (2010): 6747-6759.

35. Wu, Guohui, et al. "Remotely triggered liposome release by near-infrared light absorption via hollow gold nanoshells." Journal of the American Chemical Society 130.26 (2008): 8175-8177.

36. Cochran, Michael C., et al. "Disposition of ultrasound sensitive polymeric drug carrier in a rat hepatocellular carcinoma model." Academic radiology18.11 (2011): 1341-1348.

37. Lin, Chung-Yin, et al. "Enhancement of focused ultrasound with microbubbles on the treatments of anticancer nanodrug in mouse tumors."Nanomedicine: Nanotechnology, Biology and Medicine 8.6 (2012): 900-907..

38. Lim, Eun-Kyung, et al. "pH-Triggered Drug-Releasing Magnetic Nanoparticles for Cancer Therapy Guided by Molecular Imaging by MRI." Advanced Materials 23.21 (2011): 2436-2442.

39. Putnam, David, and Jindrich Kopecek. "Enantioselective release of 5-fluorouracil from $\mathrm{N}$-(2-hydroxypropyl) methacrylamide-based copolymers via lysosomal enzymes." Bioconjugate chemistry 6.4 (1995): 483-492.

40. Putnam, D., and J. Kopeček. "Polymer conjugates with anticancer activity."Biopolymers li. Springer Berlin Heidelberg, 1995. 55-123.

41. Wong, Cliff, et al. "Multistage nanoparticle delivery system for deep penetration into tumor tissue." Proceedings of the National Academy of Sciences 108.6 (2011): 24262431.

42. Brannon-Peppas, Lisa, and James O. Blanchette. "Nanoparticle and targeted systems for cancer therapy." Advanced drug delivery reviews 64 (2012): 206-212. 
43. Rodzinski, Alexandra, et al. "Targeted and controlled anticancer drug delivery and release with magnetoelectric nanoparticles." Scientific reports 6 (2016).

44. Nair, Madhavan, et al. "Externally controlled on-demand release of anti-HIV drug using magneto-electric nanoparticles as carriers." Nature communications 4 (2013): 1707.

45. Yue, Kun, et al. "Magneto-electric nano-particles for non-invasive brain stimulation." PloS one 7.9 (2012): e44040.

46. Guduru, Rakesh, et al. "Magneto-electric nanoparticles to enable field-controlled highspecificity drug delivery to eradicate ovarian cancer cells."Scientific reports 3 (2013).

47. Guduru, Rakesh, and Sakhrat Khizroev. "Magnetic Field-Controlled Release of Paclitaxel Drug from Functionalized Magnetoelectric Nanoparticles."Particle \& Particle Systems Characterization 31.5 (2014): 605-611.

48. Binggeli, Richard, and Ivan L. Cameron. "Cellular potentials of normal and cancerous fibroblasts and hepatocytes." Cancer research 40.6 (1980): 1830-1835.

49. Cahill, Kevin. "Molecular electroporation and the transduction of oligoarginines." Physical biology 7.1 (2009): 016001.

50. Redmann, K., et al. "The membrane potential of primary ovarian tumor cells in vitro and its dependence on the cell cycle." Acta biologica et medica Germanica 28.5 (1972): 853.

51. Armstrong, Deborah K., et al. "Intraperitoneal cisplatin and paclitaxel in ovarian cancer." New England Journal of Medicine 354.1 (2006): 34-43.

52. Kim, D-W., et al. "Multicenter phase II trial of Genexol-PM, a novel Cremophor-free, polymeric micelle formulation of paclitaxel, with cisplatin in patients with advanced nonsmall-cell lung cancer." Annals of oncology18.12 (2007): 2009-2014.

53. Parkin, Stuart, et al. "Magnetically engineered spintronic sensors and memory." Proceedings of the IEEE 91.5 (2003): 661-680.

54. Tsoi, M., et al. "Excitation of a magnetic multilayer by an electric current."Physical Review Letters 80.19 (1998): 4281.

55. Slonczewski, J. C. "Currents and torques in metallic magnetic multilayers."Journal of Magnetism and Magnetic Materials 247.3 (2002): 324-338.

56. Berger, L. "Emission of spin waves by a magnetic multilayer traversed by a current." Physical Review B 54.13 (1996): 9353.

57. Pushp, Aakash, et al. "Giant thermal spin-torque-assisted magnetic tunnel junction switching." Proceedings of the National Academy of Sciences112.21 (2015): 6585-6590. 
58. Safonov, Vladimir L. Nonequilibrium magnons: Theory, experiment and applications. John Wiley \& Sons, 2012.

59. Hong, Jeongmin, et al. "Energy-efficient spin-transfer torque magnetization reversal in sub-10-nm magnetic tunneling junction point contacts." Journal of nanoparticle research 15.4 (2013): 1-6.

60. Hong, Jeongmin, et al. "The Physics of Spin-transfer Torque Switching in Magnetic Tunneling Junctions in Sub-10-nm Size Range."

61. Stone, Mark, et al. "Anomalous properties of sub-10-nm magnetic tunneling junctions." Energy Efficient Electronic Systems (E3S), 2015 Fourth Berkeley Symposium on. IEEE, 2015. 
Hypothesis and research goals

\section{Nanomedicine}

\section{Research goals}

In order to tailor a carrier for cancer medication, having a complete understanding of the metabolism and behavior of magnetoelectric nanoparticles (MENs) is crucial. This study aims to address this need through an in-vivo study of particles in the blood stream and vital organs. A mouse model has been implemented to perform the study.

\section{Research outline}

This section of the research includes the following stages:

1. MENs characterization and injection 2. euthanization and sample preparation for SEM imaging , 3. SEM imaging and 4. image analysis, standardization, and data analysis of the results.

\section{MENs characterization and injection}

Different sizes $(10 \mathrm{~nm}, 30 \mathrm{~nm}, 100 \mathrm{~nm}, 600 \mathrm{~nm})$ coreshell MENs are characterized using transmission electron microscopy (TEM). Their magnetic field dependence on zeta potential was measured for $0.5 \mathrm{mg}$ of MENs. Magnetic force microscopy (MFM) confirmed their dipole nature. After the characterization was complete, the MENs are administrated through systemic IV injection into the lateral tail vein of a mouse. 
The list of samples to be studied:

Table 2.1. The list of samples for bio-distribution study

\begin{tabular}{|c|c|c|c|}
\hline & 1 week post injection & 4 weeks post injection & 8 weeks post injection \\
\hline \multirow{4}{*}{ Brain } & & & 10nm particles \\
\hline & & & 30nm Particles \\
\hline & & & 100nm Particles \\
\hline & $600 \mathrm{~nm}$ Particles & 600 nm Particles & 600 nm Particles \\
\hline \multirow{4}{*}{ Liver } & 10nm particles & 10nm particles & 10nm particles \\
\hline & 30nm Particles & 30nm Particles & 30nm Particles \\
\hline & 100nm Particles & 100nm Particles & 100nm Particles \\
\hline & 600 nm Particles & 600 nm Particles & 600 nm Particles \\
\hline \multirow{4}{*}{ Lung } & 10nm particles & 10nm particles & 10nm particles \\
\hline & 30nm Particles & 30nm Particles & 30nm Particles \\
\hline & 100nm Particles & 100nm Particles & 100nm Particles \\
\hline & 600 nm Particles & 600 nm Particles & 600 nm Particles \\
\hline \multirow{4}{*}{ Spleen } & 10nm particles & 10nm particles & 10nm particles \\
\hline & 30nm Particles & 30nm Particles & 30nm Particles \\
\hline & 100nm Particles & 100nm Particles & 100nm Particles \\
\hline & 600 nm Particles & 600 nm Particles & 600 nm Particles \\
\hline \multirow{4}{*}{ Kidney } & 10nm particles & 10nm particles & 10nm particles \\
\hline & 30nm Particles & 30nm Particles & 30nm Particles \\
\hline & 100nm Particles & 100nm Particles & 100nm Particles \\
\hline & 600 nm Particles & 600 nm Particles & 600 nm Particles \\
\hline
\end{tabular}




\section{Euthanization and sample preparation for SEM imaging}

Different time intervals after the injection (24 hours, 1 week, 4 weeks and 8 weeks post injection) were investigated in order to achieve a comprehensive understanding of the metabolism of the nanoparticles with different sizes. Vital organs (brain, lung, liver and spleen) were harvested after these time intervals from the mice injected with different nanoparticle sizes. The slides which were prepared from these organs were placed on microscope slides and prepared for the SEM imaging.

\section{SEM imaging}

Samples were coated, grounded, and loaded into a JEOL JIB 4500 equipped with a backscattered electron detector, which was then used to locate the clusters. The final confirmation that the cluster sites were actually MENs was done by point and shoot and mapping energy dispersive spectroscopy (EDS) of the sites located by the backscattered electron detector.

\section{Image analysis, standardization, and data analysis of the results}

Around $10 \mathrm{~Gb}$ of data including images, EDS mappings, and point and shoots were collected. For each organ, each time interval and each particle size images which were indicative of the distribution were chosen. Using imagej software, the distribution in a specific area of the tissue was calculated and averaged.

\section{Nanoelectronics}

This research aims to investigate the validity of the following hypothesis: 


\section{Hypothesis}

In a sub-10nm magnetic tunneling junction (MTJ), a switching current reduction superior to that of a reduction because of a linear area scaling can be expected.

The thermal reservoir is usually responsible for absorbing the energy of excitations. In this size range, the thermal reservoir becomes extremely small. Hence, it becomes unable to absorb the energy which was produced from the collective spin dynamics [1]. Additionally, in this size range it is inappropriate to use the continuous crystalline lattice model. A more suitable model for this range is a cluster where the energy transfer between the excitations is less effective. The aforementioned parameters result in a large magnetization damping decrease that in turn causes a decrease in the energy required for the switching [2].

\section{Research goals}

the goal of this study is to fabricate a sub $10 \mathrm{~nm}$ magnetic tunneling junction using lithography techniques and a focused ion beam in order to investigate the hypothesis.

\section{Research outline}

The plan to test the validity of the hypothesis includes the following stages:

1. MTJ fabrication in the cleanroom, 2. Quality control testing of the fabricated junctions, 3. Focused lon beam trimming, and 4. Characterization and magnetic measurement of the MTJs.

\section{MTJ fabrication in the cleanroom}

After proposing several sketches of the MTJs, a design, which was more suitable for further trimming with FIB, was chosen. Then a mask was fabricated using "Layout Editor" software. The produced mask was employed for fabricating the MTJs by lithography. An ATC 1800 ORION magnetron sputtering system from AJA international was used to sputter the popular perpendicular MTJ multilayer composition, which is $\mathrm{Ta}(5 \mathrm{~nm}) / \mathrm{CoFeB}(1$ 
$\mathrm{nm}) / \mathrm{MgO}(0.9 \mathrm{~nm}) / \mathrm{CoFeB}(1 \mathrm{~nm}) / \mathrm{Ta}(5 \mathrm{~nm})$. All the layers were deposited under a $10 \mathrm{mTorr}$ argon pressure with a sample rotation to assure the uniform coverage of the sample.

\section{Quality control testing of the fabricated junctions}

The composition has to provide an optimal combination of properties such as high spin torque efficiency, high magnetoresistance, and ultra-high interfaceinduced perpendicular anisotropy energy. The interface perpendicular anisotropy field was measured by deploying a focused magneto kerr effect. Magnetoresistance was measured before and after the FIB trimming to remove the possibility of creating shorts. Atomic force microscopy (AFM) and magnetic force microscopy (MFM) measurements confirmed the deposition of magnetic layers on the wafer.

\section{Focused Ion beam trimming}

After the samples were fabricated, they were loaded into a JEOL JIB4500 dual beam focused ion beam in order to be trimmed. At first coarser beam currents ( 100 pA) were employed for the coarser cuts and then as the size of the junction decreased finer beam currents $(\sim<10 \mathrm{pA})$ were used in order to protect the sample from over exposure to the ion beam.

\section{Characterization and magnetic measurement of the MTJs}

Next stage of the study consisted of scanning electron microscopy imaging for confirmation and measurement of the junction area, transmission electron microscopy (TEM) analysis and the I-V swipes for measuring the magnetoresistance. The last stage of the study, after identification of a MTJ with a proper magnetoresistance, was measuring the switching current. 


\section{References:}

1. Hong, Jeongmin, et al. "Energy-efficient spin-transfer torque mgnetization reversal in sub-10-nm magnetic tunneling junction point contacts." Journal of nanoparticle research 15.4 (2013): 1-6.

2. Hong, Jeongmin, et al. "The Physics of Spin-transfer Torque Switching in Magnetic Tunneling Junctions in Sub-10-nm Size Range." 


\section{Experimental}

\section{Nanomedicine}

\section{Fabrication of MENs particles}

Details of the fabrication of toxicity-free MENs have been explained in a previous publication [1]. To give a brief description, the basic structure of CoFe2O4-BaTiO3 coreshell MENs was synthesized according to the following steps:

(i) $0.058 \mathrm{~g}$ of $\mathrm{Co}(\mathrm{NO} 3) 2 \cdot 6 \mathrm{H} 2 \mathrm{O}$ and $0.16 \mathrm{~g}$ of $\mathrm{Fe}(\mathrm{NO} 3) 3.9 \mathrm{H} 2 \mathrm{O}$ were dissolved in $15 \mathrm{~mL}$ of deionized (DI) water; (ii) $5 \mathrm{~mL}$ of aqueous solution containing $0.9 \mathrm{~g}$ of sodium borohydride and $0.2 \mathrm{~g}$ of polyvinylpyrrolidone was added at $120^{\circ} \mathrm{C}$ for $12 \mathrm{~h}$ to obtain CoFe2O4 nanoparticles; (iii) BaTiO3 precursor solution was prepared by adding $30 \mathrm{~mL}$ of DI water containing $0.029 \mathrm{~g}$ of $\mathrm{BaCO} 3$ and $0.1 \mathrm{~g}$ of citric acid to $30 \mathrm{~mL}$ ethanolic solution containing $1 \mathrm{~g}$ of citric acid and $0.048 \mathrm{~mL}$ of titanium (IV) isopropoxide; (iv) The prepared CoFe2O4 nanoparticles $(0.1 \mathrm{~g})$ were added to the $60 \mathrm{~mL}$ of BaTiO3 precursor solution and sonicated for $120 \mathrm{~min}$; (v) The resulted dispersed nanoparticles were dried on hot plate at $60^{\circ} \mathrm{C}$ for $12 \mathrm{~h}$, while stirring at $200 \mathrm{rpm}$; (vi) The nanoparticle precursor was heated and cooled in a box-furnace. The temperature and timing of the heating and cooling cycle determined the size of the particles.

\section{MENs surface modification}

The fabricated nanoparticles were then surface functionalized by a 2-nm thick coating of glycerol monooleate. glycerol monooleate-MENs were prepared according to the method described by Guduru, R. et al. by incubating $0.1 \mathrm{mg}$ of glycerol monooleate with $5 \mathrm{mg}$ of MENs in $5 \mathrm{~mL}$ of PBS buffer which has a $\mathrm{pH}$ of 7.4 for 12 hours. The solution was slowly agitated during incubation in order to obtain uniform surface modification. The solution was then centrifuged at $20000 \mathrm{rpm}$ for $20 \mathrm{~min}$ at $10{ }^{\circ} \mathrm{C}$. The step was carried out to 
eliminate excess glycerol monooleate. The pellet which was obtained was suspended again in ethyl acetate:acetone (70:30) solution and centrifuged three times to obtain glycerol monooleate -MENs. Surface-modified MENs were lyophilized and stored at $4{ }^{\circ} \mathrm{C}$ until further use. The particle characterization was carried out using scanning electron microscopy, transmission electron microscopy and energy dispersive spectroscopy.
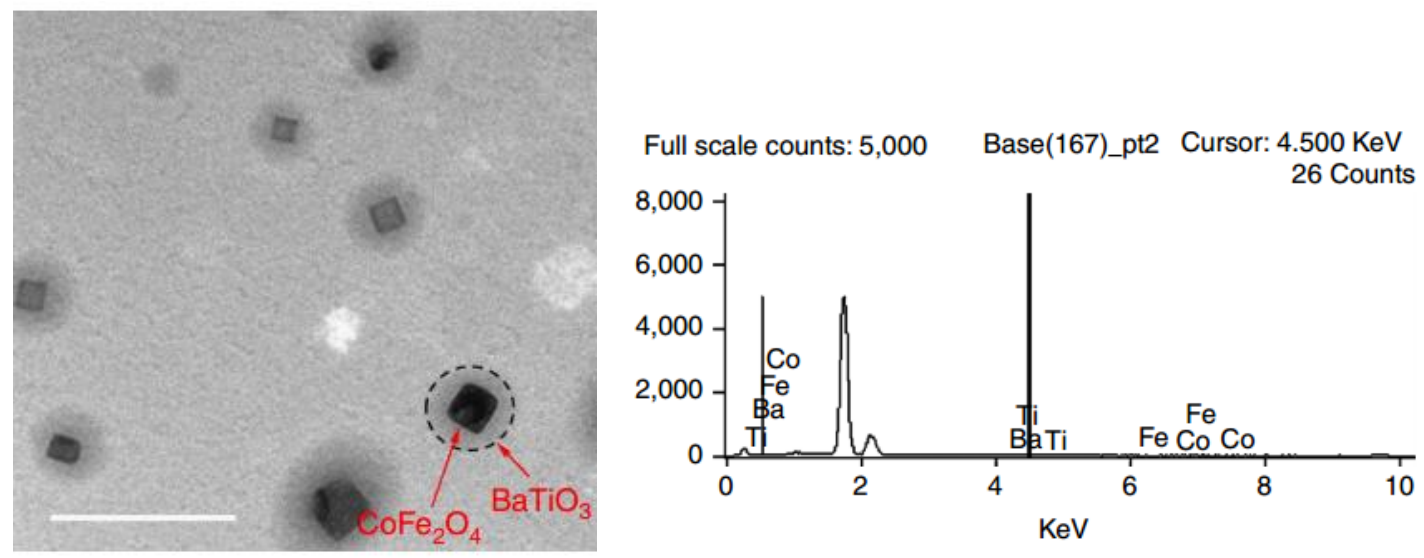

Figure 3.1. TEM image of the MENs on the left and the EDS data gathered from SEM on the right [2]

\section{Animals}

All the methods used in this study were carried out in accordance with the approved guidelines of the institutional animal care and use committee (IACUC) document \# 13-045 at Florida International University (FIU).

This study used immunodeficient ICR scid mice from Taconic in order to be comparable to the immunodeficient mice used in the previous cancer study [3]. The mice were maintained under a specific pathogen-free environment in the institutional animal care facility at all times. The mice were placed under isoflurane anesthesia and injected with a single dose of $5 \mathrm{mg}$ GMO MENs in $250 \mu \mathrm{L}$ PBS in the lateral tail vein. Animals were sacrificed for organ harvest after the specific time intervals of 24 hours, 1 week, 1 month, and 2 months. 


\section{Tissue Preparation}

Mice were euthanized by means of $\mathrm{CO} 2$ inhalation and the organs of interest were excised immediately for storage in a $10 \%$ formalin solution overnight at $4{ }^{\circ} \mathrm{C}$. After any necessary cleaning under a stereomicroscope, a small piece of the tissue was cut and transferred into PBS, where it was rocked for 30 minutes with the PBS being replaced 3 times in 10 minute intervals to remove excess fixative. Tissues were carefully dried with a Kimwipe and transferred to a plastic mold containing OCT (optimal cutting temperature medium). Tissues were frozen either by immersing the mold directly into liquid nitrogen or in a bath of 2-methylbutane/liquid nitrogen at approximately $-100^{\circ} \mathrm{C}$, depending on the type of tissue being processed. Frozen tissues were transferred to a $-80^{\circ} \mathrm{C}$ freezer for at least one night before being cut into $10 \mu \mathrm{m}$ sections with a Leica CM3050 Cryostat. The tissue slices were mounted on $\mathrm{VWR}^{\circledR}$ Superfrost ${ }^{\circledR}$ Plus microscope slides and dried on a slide warmer at $37^{\circ} \mathrm{C}$ for $1-2$ hours. Any extra OCT was rinsed off the slides using PBS prior to gold sputtering.

\section{Particle detection}

An important issue to be addressed in this study was finding a way to systematically trace and locate the particles in the tissue once the organs have been harvested. The image formed by the secondary electron detector is dominated by the topography of the sample

(in this case tissue) and the particles we are looking for are in the order of nanometers (if they are clustered, maybe a few microns). These particles are hard to locate by their shape so using the secondary electron detector is not going to be appropriate for this case. The 
backscattered electron detector, on the other hand, screens for the particles based on their atomic weight.

An area of the sample which has a lower atomic weight will not emit as many particles as the high atomic weight area will. The tissue is mainly composed of carbon and organic materials, while the particles are composed of relatively heavy elements. This makes the backscattered detector an ideal tool for locating the particles in the tissue, as can be seen in Figure 3.1
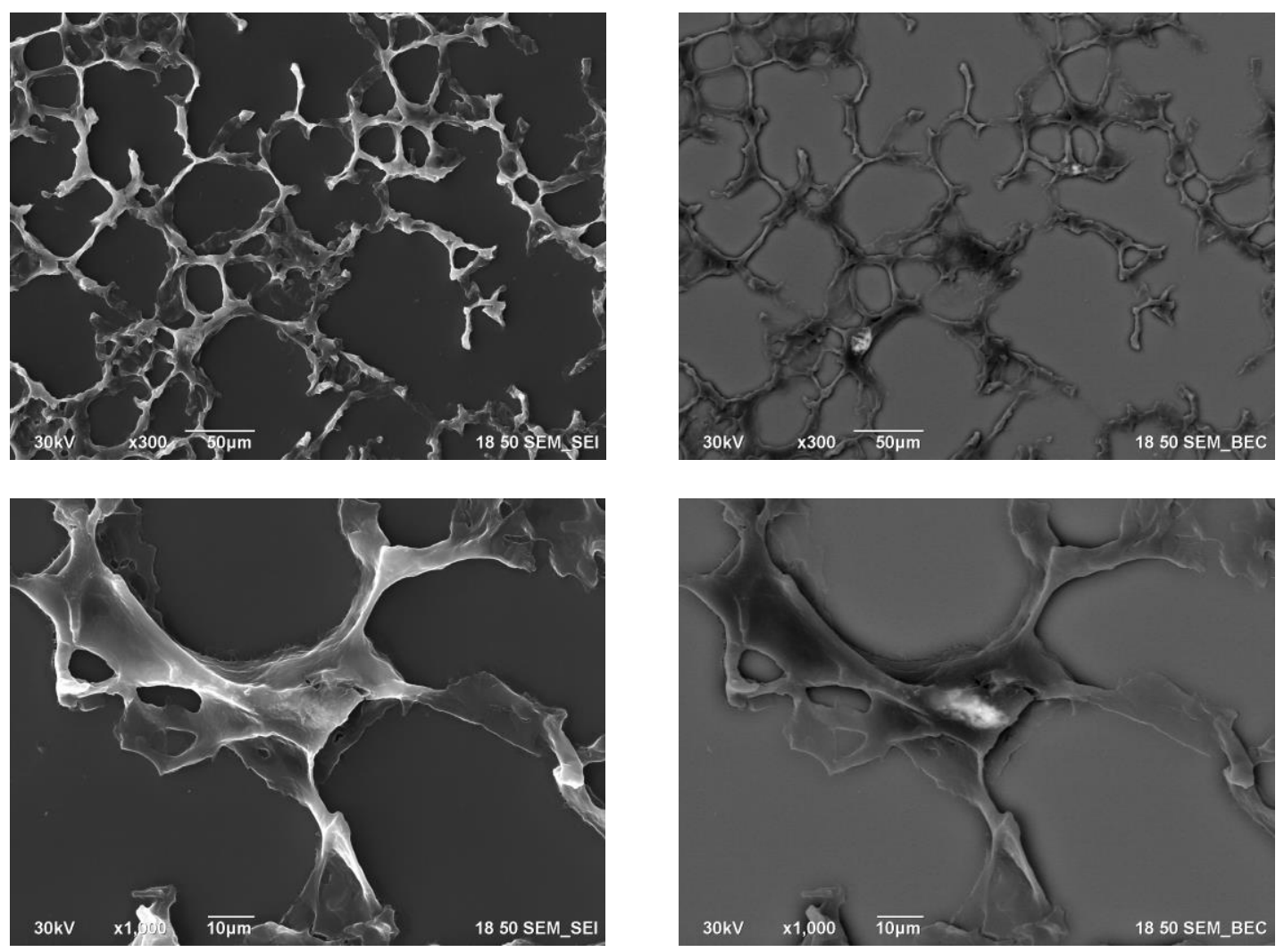

Figure 3.2. Secondary image vs backscattered image

On the left side both images show a secondary electron image of an area in the lung and on the right both images show the same area imaged by backscattered detector where the particles are shining in the images

It is worthy of noting that some organs have plenty of other minerals which are relatively heavy such as iron and calcium. Hence, after detection of a high contrast area it was crucial to confirm that the detected area contained Titanium and Barium using the EDS 
detector. Since these elements are rarely found in body, detection of these elements by EDS can confirm that the detected area indeed contained the particles.
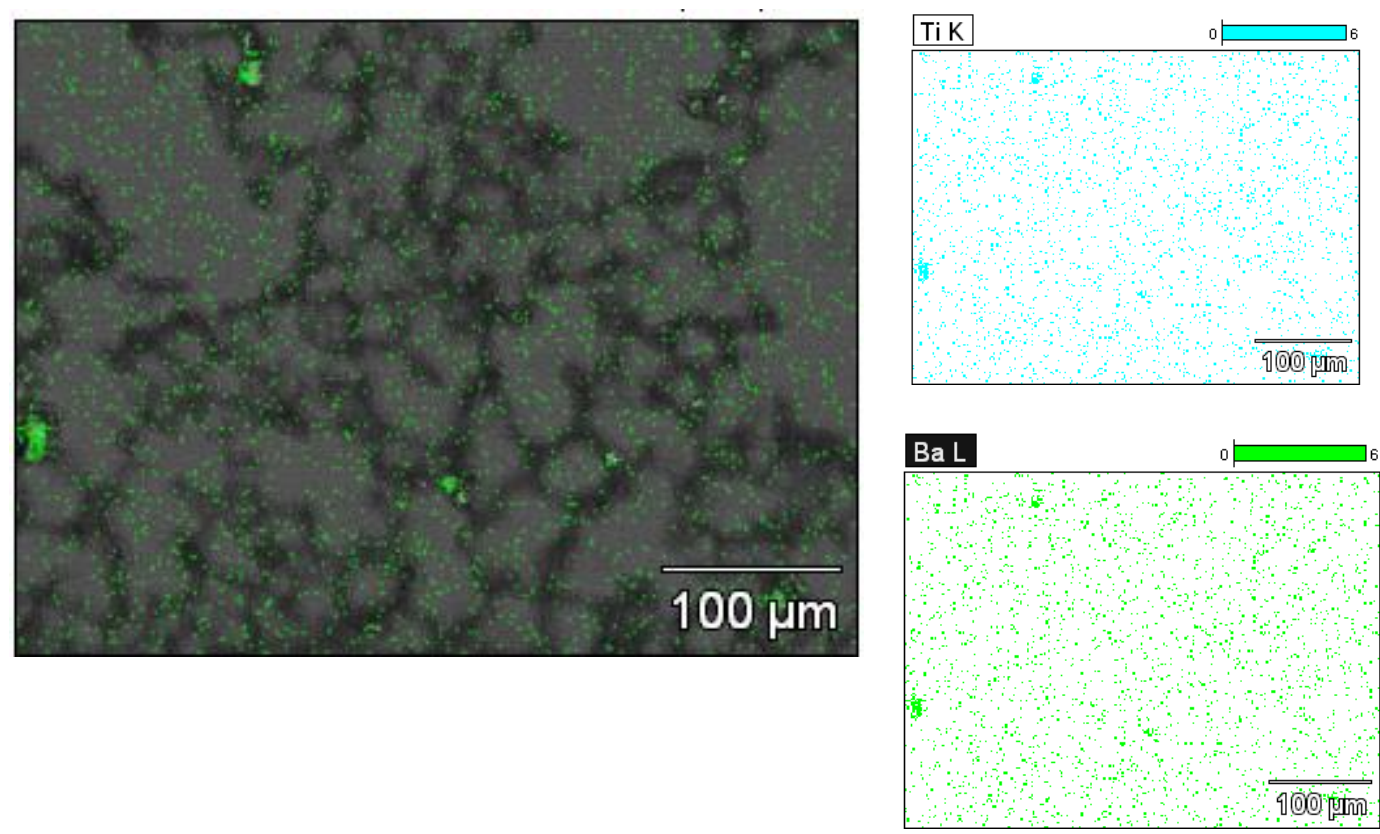

Figure 3.3. Backscattered image \& mapping EDS of an area in the lung 1 month post injection

\section{Image processing and analysis}

Approximately $10 \mathrm{~GB}$ of data including images and EDS results were collected. This data needs to be processed before any conclusion about the bio-distribution of the particles can be drawn. "Imagej" software was used in order to perform the image analysis. For each organ, each time lapse, and each particle size, the images were compared to their corresponding EDS results. The images which were approximately indicative of the distribution of the particles of that size in that specific time and for that specific organ were chosen. Then, the chosen images were scaled using the scale bar and processed by using a threshold and masking the whole image except for the areas containing the particles. 
Then, the distribution of the particle were calculated over the area which was selected. This process was repeated for a few images of each category and then they were averaged to yield a more reliable result for the distribution of the particles in that certain organ in that time period.
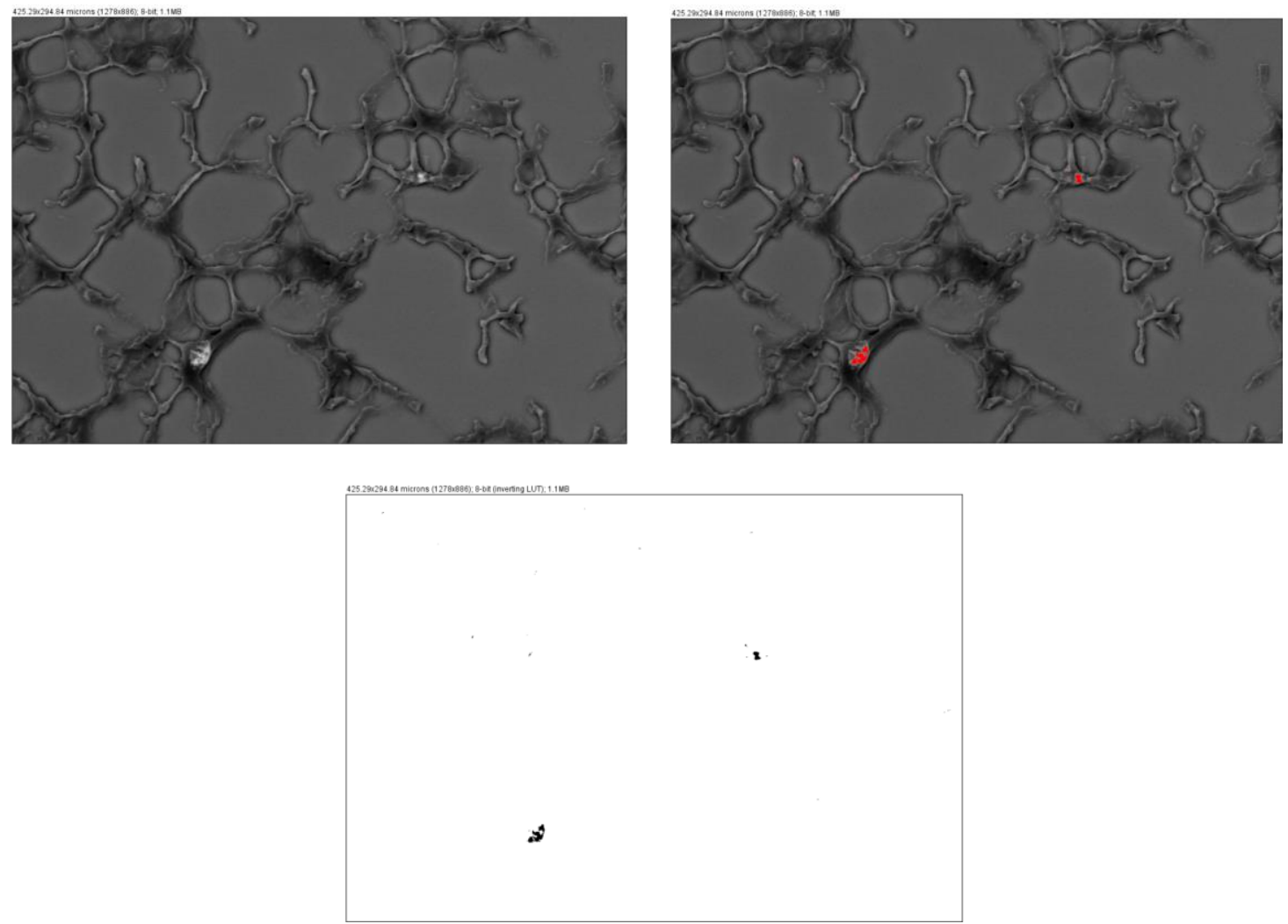

Figure 3.4. Different stages of image analysis

Top left image shows the BEC image that was picked for the analysis. Top right image shows the masked area using a contrast threshold and the bottom area shows the particles which were picked by the software for the image analysis

\section{Nanoelectronics}

The study was performed in different phases and in each, a new generation of the MTJs were fabricated. 


\section{First generation of the devices}

In phase one, which was is the preliminary study, the design was prepared at FIU and the MTJs were fabricated at the cleanroom by photolithography.

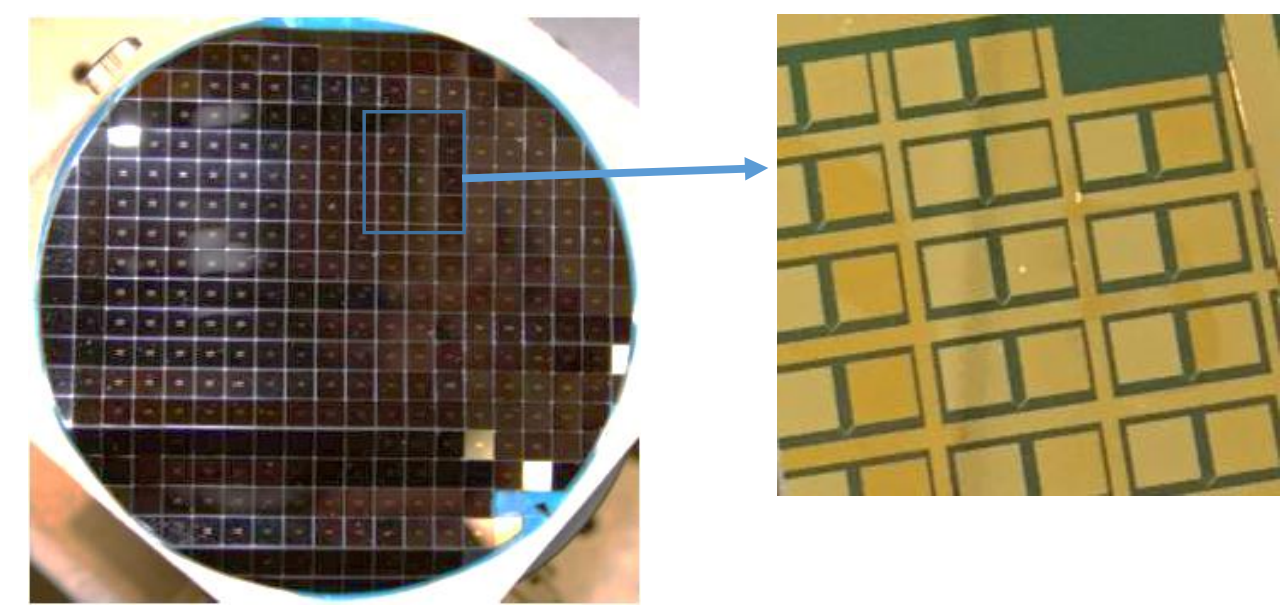

Figure 3.5. MTJs fabricated in phase one

Over 140 MTJ devices were fabricated at this stage by photolithography. Each device was then inspected for defects and was loaded into a dual beam JEOL-JIB 4500 focused ion beam platform. Using the SEM, these samples were inspected for possible opens or shorts. Then, using the $\mathrm{Ga}$ ion beam at $30 \mathrm{KeV}$, they were milled down to fabricate MTJ devices ranging between $24-100 \mathrm{~nm}$. Due to the large area of the milling at the initial stages, high current beams around 100pA were employed at first. As the junction became smaller the beam current was reduced in order to prevent overexposure. The final beam current which was used was $\sim<10 \mathrm{pA}$. These samples were then characterized using a JEOL 6330 field emission scanning electron microscope at $30 \mathrm{Kev}$ and $12 \mu \mathrm{A}$ probe current and tunneling microscopy. Magnetoresistance measurements paved the path for better understanding the magnetic behavior and measuring the switching current density in this region. 


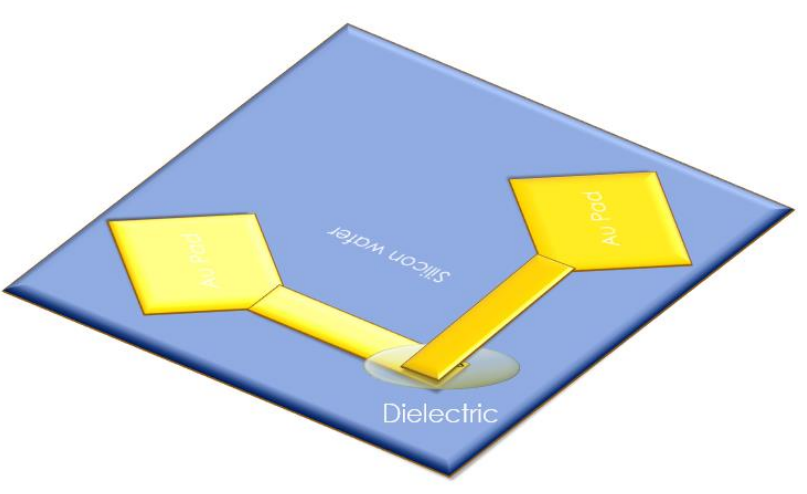

Figure 3.6. Schematic illustration

First generation Junction on the left and SEM image of a trimmed MTJ on the right

\section{Second generation of the devices}

The first set of samples had a couple of issues which needed to be addressed. These samples sometimes exhibited significant magnetic instability due to the field induced motion of single domains through overlapping surfaces. Additionally, this design suffered from a few flaws. There were areas in which the junction was not protected during the ion bombardment. Leaving areas of the MTJ exposed can change the result of the magnetic measurements. Furthermore, the MgO deposition was not uniform all over the surface. This can intensely affect the tunneling current and can bias the results. In order to refine the measurement results, another set of MTJs was developed. "Layout editor" software was used in order to design the devices. After deciding the best parameters for the pads, the junction, and a dielectric, these new MTJs were fabricated by sputter deposition in the Florida International University cleanroom. In the new set of samples, the aforementioned issues were addressed, allowing for accurate measurements and exact quantitative analysis of the MTJs magnetic response at the sub $10 \mathrm{~nm}$ region. 


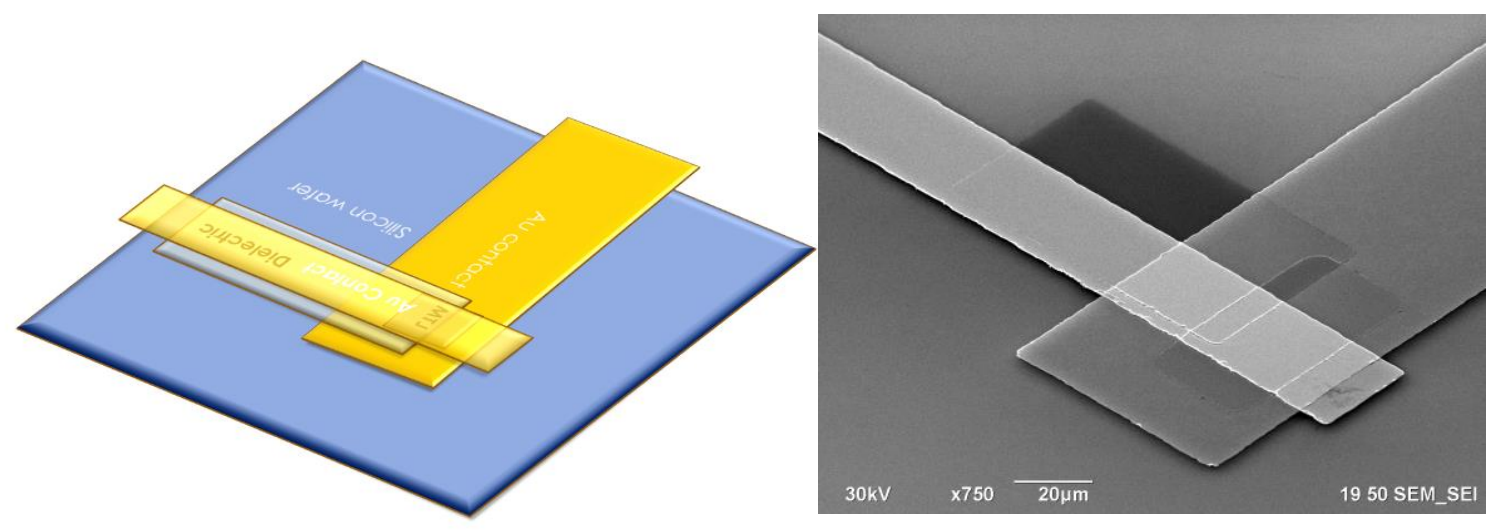

Figure 3.7. Schematic illustration

Second generation Junction on the left and SEM image of the MTJ on the right

Uniformity of the sputtered MgO layer was confirmed by TEM analysis. A JEOL JIB-4500 Multi-beam Focused Ion Beam with a Gallium ion source at 30KV was employed to mill out thin foils of the samples (lamella) for TEM analysis. These lamellae were then lifted out and were put on TEM grids. TEM imaging was performed by a Phillips CM-200 at 200 $\mathrm{KV}$ operating voltage. After this stage, the samples were loaded into a JEOL JIB 4500 dual beam FIB. The samples were milled down using beam currents ranging from 100pA to $<10 \mathrm{~Pa}$ for the final cuts. The smallest structure that was fabricated with this method was approximately $23 \mathrm{~nm}$. This seems to be the smallest structure which can be fabricated by Ga ion FIB due to its limited resolution. In order to conduct a comprehensive study at sub 10nm range, a collaboration between FIU and UC-Berkeley was formed in order to take advantage of the state of the art Helium ion microscope (HIM). This HIM is equipped with $\mathrm{Ga}, \mathrm{Ne}$, and $\mathrm{He}$ and has been designed specifically for the creation of sub $10 \mathrm{~nm}$ structures. The Orion nanofab helium ion microscope also has an imaging resolution of $0.5 \mathrm{~nm}$. Additionally, HIMs usually have 5 to 10 times depth of field of a field emission scanning electron microscope. 


\section{References:}

1. Guduru, Rakesh, and Sakhrat Khizroev. "Magnetic Field-Controlled Release of Paclitaxel Drug from Functionalized Magnetoelectric Nanoparticles."Particle \& Particle Systems Characterization 31.5 (2014): 605-611.

2. Nair, Madhavan, et al. "Externally controlled on-demand release of anti-HIV drug using magneto-electric nanoparticles as carriers." Nature communications 4 (2013): 1707.

3. Rodzinski, Alexandra, et al. "Targeted and controlled anticancer drug delivery and release with magnetoelectric nanoparticles." Scientific reports 6 (2016). 


\section{Results and Discussion}

\section{Nanomedicine}

It seems that the nanoparticles get discharged systematically by both phagocytic uptake and excretion through the kidneys. However, the rate of discharge seems to be slower than initially expected. Even after two months, small remnants of the clusters could be detected in different organs. Additionally, some clusters of particles were located in organs that often do not accommodate any clusters, e.g. the brain. The schematics that follow show how the clusters of different particle sizes in different organs decrease in size and number over time.

These schematics, however, do not result in a meaningful conclusion unless there could be a way to measure the distribution over an area of organ for a specific size of MENs particles and over time. It is required for the research to have a quantitative approximation of the MENs metabolism. In order to achieve a comprehensive understanding of how these clusters change over time, the acquired images were filtered. Images which were better representative of the distribution of MENs in each organ were chosen. The particle size and distribution was then analyzed in each image by the software. The results for each specific organ and each specific particle size was averaged with the data from other images and was compared to the same organ with the same particle size at a different time point. The following tables and charts show the aforementioned process. 


\section{Lung}
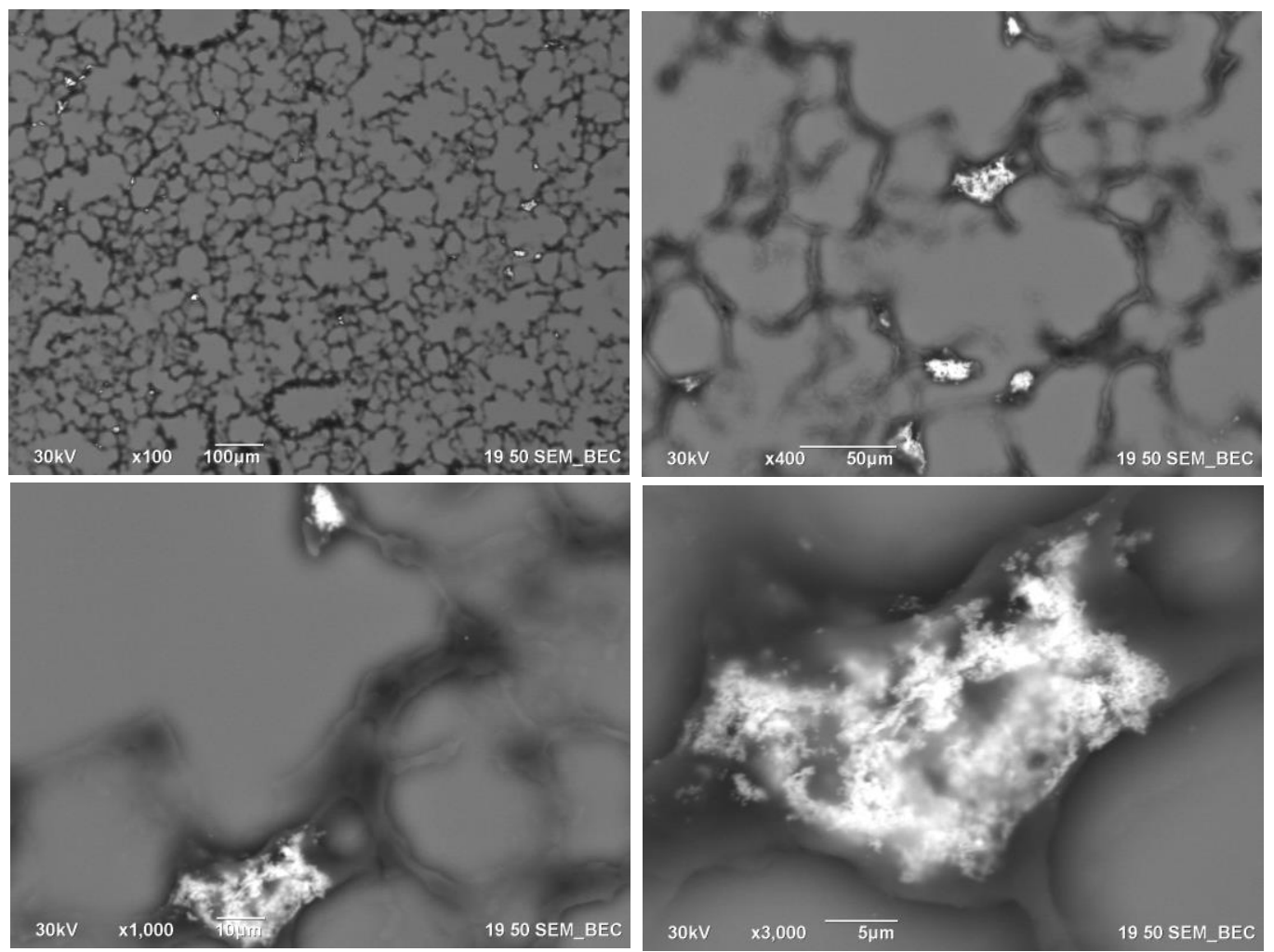

Figure 4.1. Particles in the lung Particle clusters in the lung at different magnifications (600nm-2months post injection)

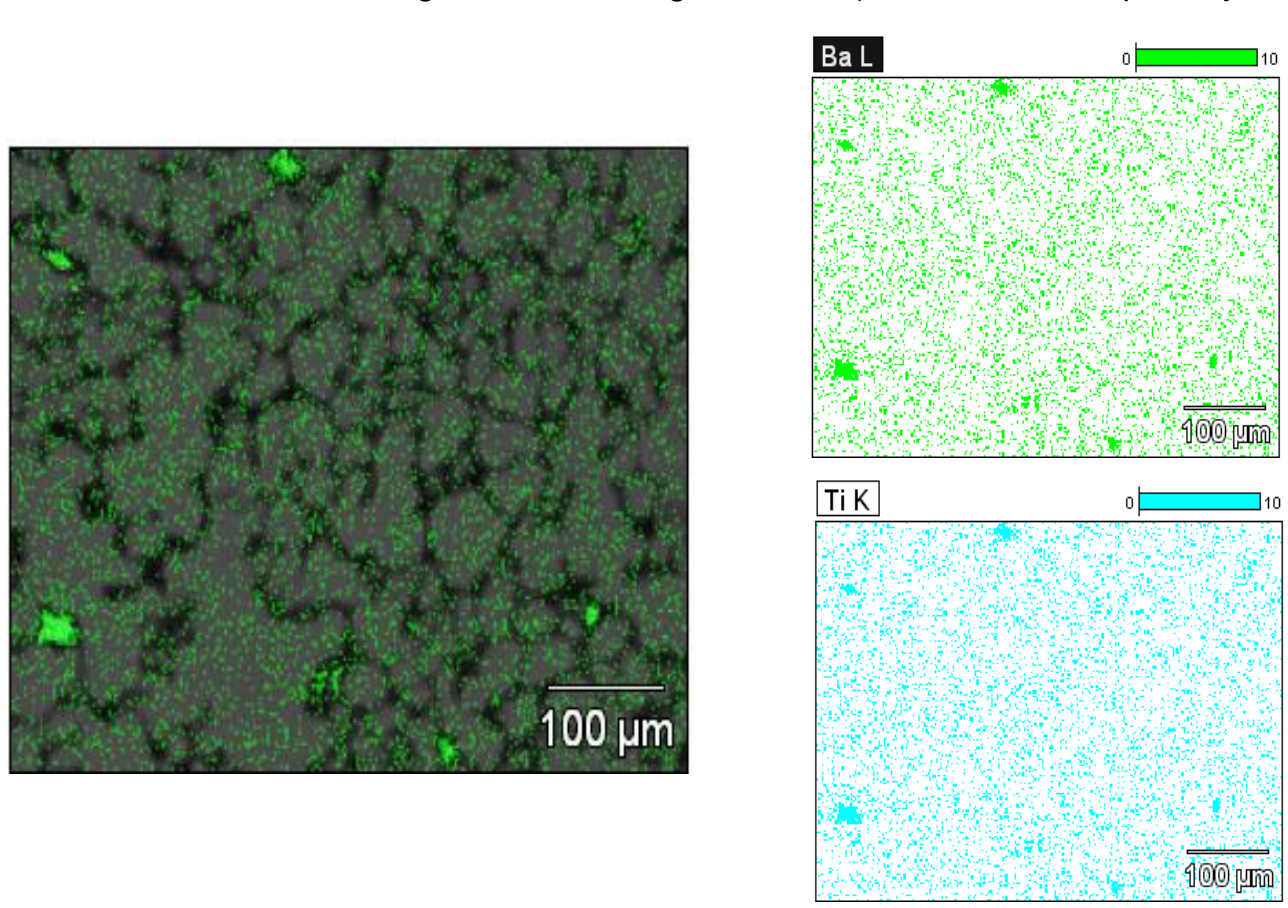

Figure 4.2. Mapping EDS of $600 \mathrm{~nm}$ MENs two months post injection in the lung 


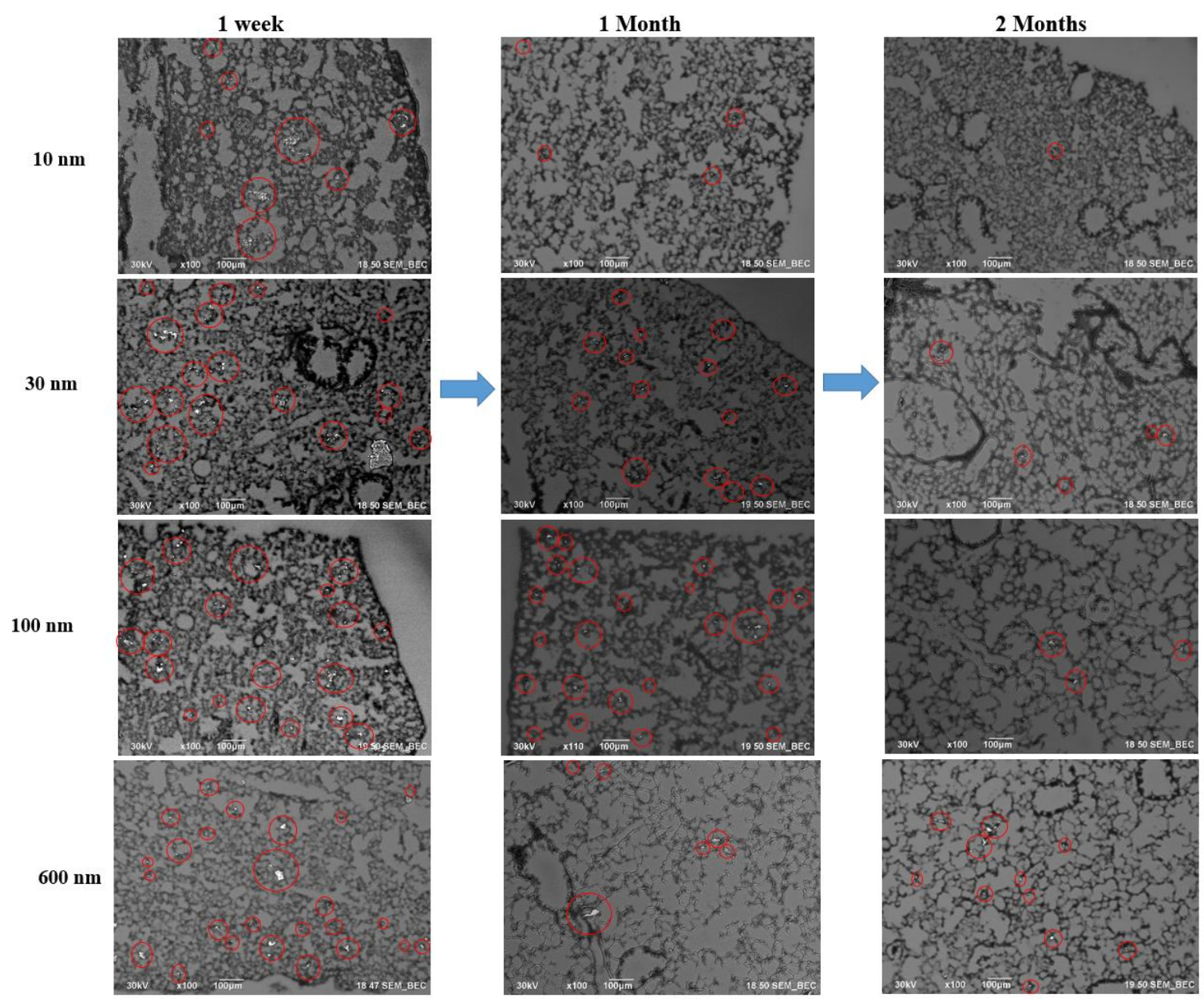

Figure 4.3. Lung schematic

Illustration of different MENs particles in the lung being discharged over time 
Table 4.1. The samples which were used for the quantitative analysis of the lung The table includes the number of the particles found by the software, the area that the particles cover, mean and standard deviation

\begin{tabular}{|c|c|c|c|c|c|}
\hline Time/ Size & Slice & count & $\%$ area & Mean \% area & STDEV \\
\hline & 100x.bmp & 87 & 0.168 & & \\
\hline \multirow[t]{3}{*}{1 week $10 \mathrm{~nm}$} & 50x.bmp & 558 & 0.142 & 0.153 & 0.0111 \\
\hline & $50 x-1 . b m p$ & 506 & 0.148 & & \\
\hline & $100 x-4 . b m p$ & 10 & 0.042 & & \\
\hline \multirow[t]{4}{*}{1 month $10 \mathrm{~nm}$} & 100x.bmp & 32 & 0.033 & 0.030 & 0.0123 \\
\hline & $100 x-9 . b m p$ & 16 & 0.034 & & \\
\hline & $100 x-2 . b m p$ & 12 & 0.009 & & \\
\hline & $100 x-2 . b m p$ & 4 & 0.003 & & \\
\hline \multirow[t]{3}{*}{2 month $10 \mathrm{~nm}$} & $100 x-3 . b m p$ & 16 & 0.004 & 0.017 & 0.0196 \\
\hline & $100 x-10 . b m p$ & 8 & 0.045 & & \\
\hline & $50 x-2 . b m p$ & 274 & 0.183 & & \\
\hline \multirow[t]{3}{*}{1 week 30nm } & 50x.bmp & 451 & 0.261 & 0.269 & 0.0733 \\
\hline & 50x.bmp & 502 & 0.362 & & \\
\hline & $100 x-16 . b m p$ & 53 & 0.066 & & \\
\hline \multirow[t]{3}{*}{1 month 30nm } & 100x.bmp & 21 & 0.079 & 0.058 & 0.0207 \\
\hline & $100 x$ around the veins.bmp & 15 & 0.03 & & \\
\hline & 50x.bmp & 44 & 0.029 & & \\
\hline \multirow[t]{3}{*}{2 month 30nm } & $50 x-7 . b m p$ & 63 & 0.047 & 0.044 & 0.0116 \\
\hline & $100 x-7 . b m p$ & 76 & 0.057 & & \\
\hline & 50x.bmp & 248 & 0.294 & & \\
\hline \multirow[t]{3}{*}{1 week 100nm } & $50 x-1 . b m p$ & 344 & 0.375 & 0.346 & 0.0366 \\
\hline & $50 x-1 . b m p$ & 279 & 0.368 & & \\
\hline & 50x.bmp & 171 & 0.151 & & \\
\hline \multirow[t]{3}{*}{1 month $100 \mathrm{~nm}$} & $50 x-1 . b m p$ & 89 & 0.086 & 0.119 & 0.0266 \\
\hline & $50 x-6 . b m p$ & 115 & 0.121 & & \\
\hline & 50x.bmp & 67 & 0.024 & & \\
\hline \multirow[t]{3}{*}{2 month $100 \mathrm{~nm}$} & $50 x-2 . b m p$ & 37 & 0.021 & 0.023 & 0.0017 \\
\hline & $50 x-7 . b m p$ & 32 & 0.025 & & \\
\hline & 50x.bmp & 119 & 0.125 & & \\
\hline \multirow[t]{3}{*}{1 week $600 \mathrm{~nm}$} & $50 x-1 . b m p$ & 156 & 0.154 & 0.133 & 0.0153 \\
\hline & $50 x-2 . b m p$ & 102 & 0.119 & & \\
\hline & 50x.bmp & 228 & 0.159 & & \\
\hline \multirow[t]{3}{*}{1 month $600 \mathrm{~nm}$} & $50 x-3 . b m p$ & 139 & 0.073 & 0.095 & 0.0457 \\
\hline & 50x-11.bmp & 20 & 0.054 & & \\
\hline & 50x.bmp & 45 & 0.065 & & \\
\hline \multirow[t]{2}{*}{2 month $600 \mathrm{~nm}$} & $50 x-2 . b m p$ & 42 & 0.059 & 0.071 & 0.0130 \\
\hline & $50 x-3 . b m p$ & 34 & 0.089 & & \\
\hline
\end{tabular}


Particle distribution over time in Lung

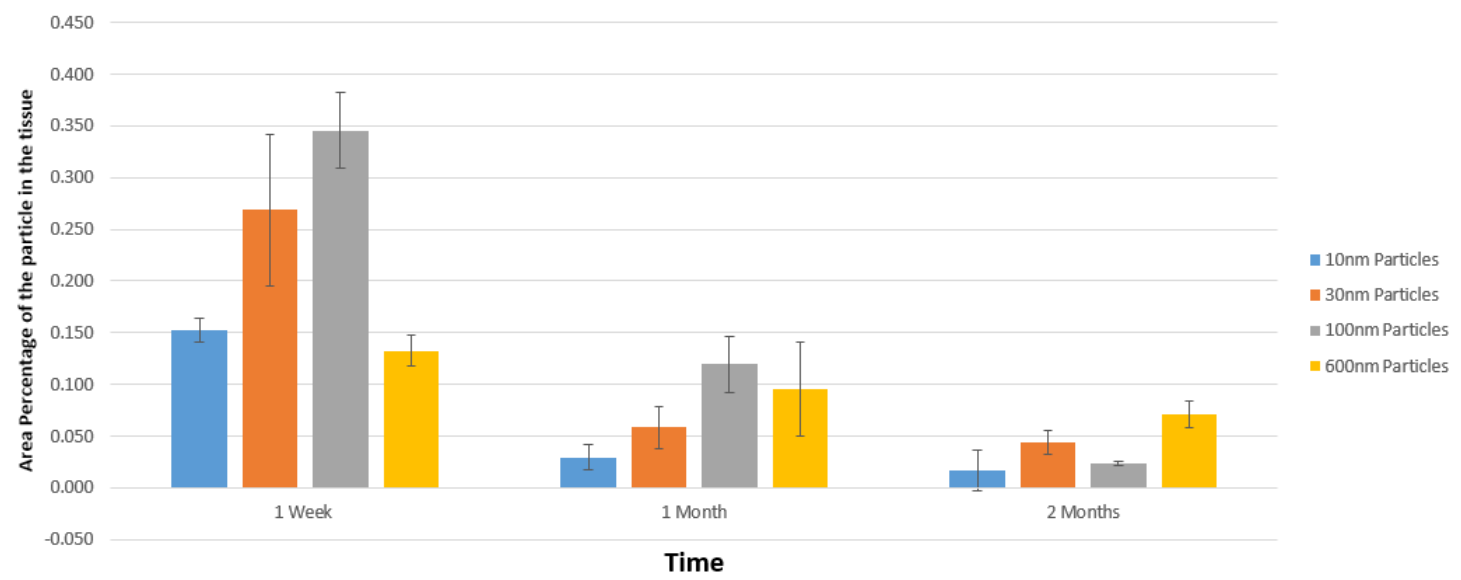

Figure 4.4. Lung distribution chart

Illustration of different size MENs particles in the lung being discharged over time

\section{PARTICLE DISTRIBUTION OVER TIME}

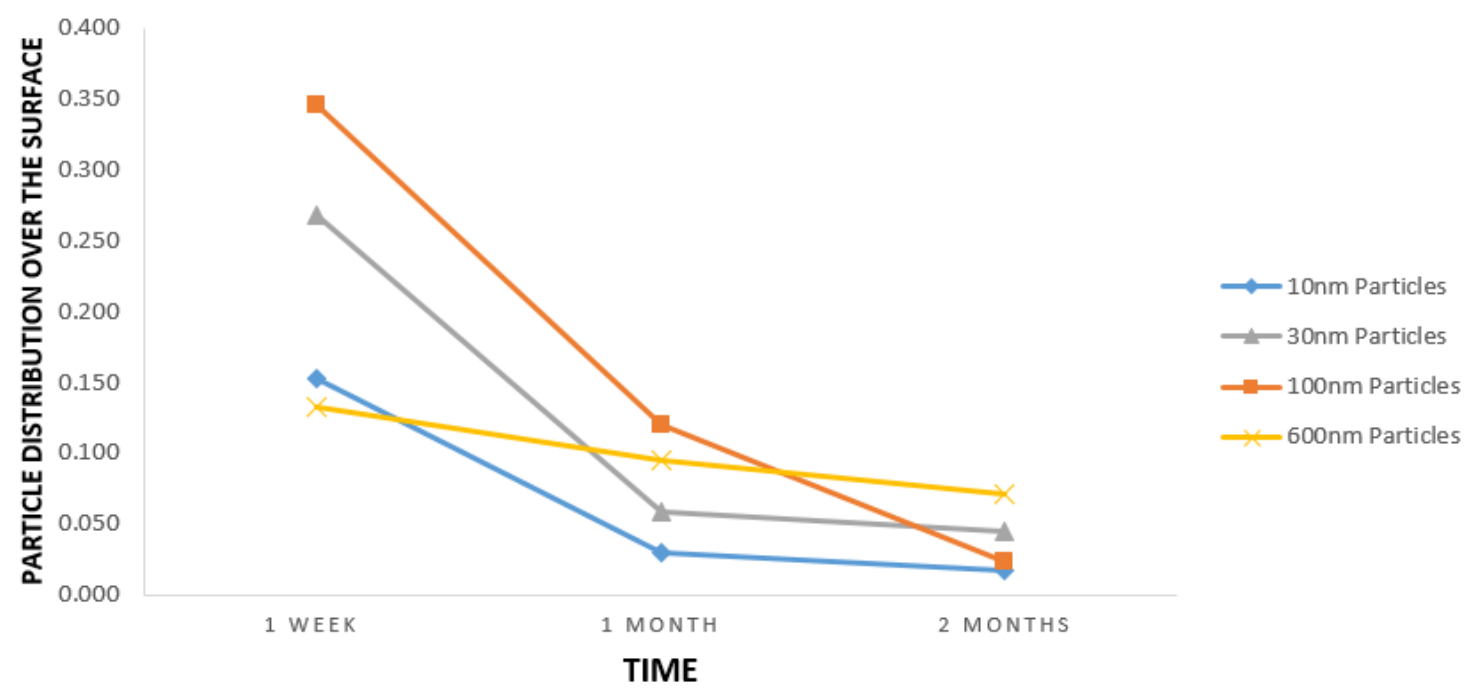

Figure 4.5. Lung distribution graph

Areal distribution of different sizes of MENs particles in the lung over time 


\section{Liver}
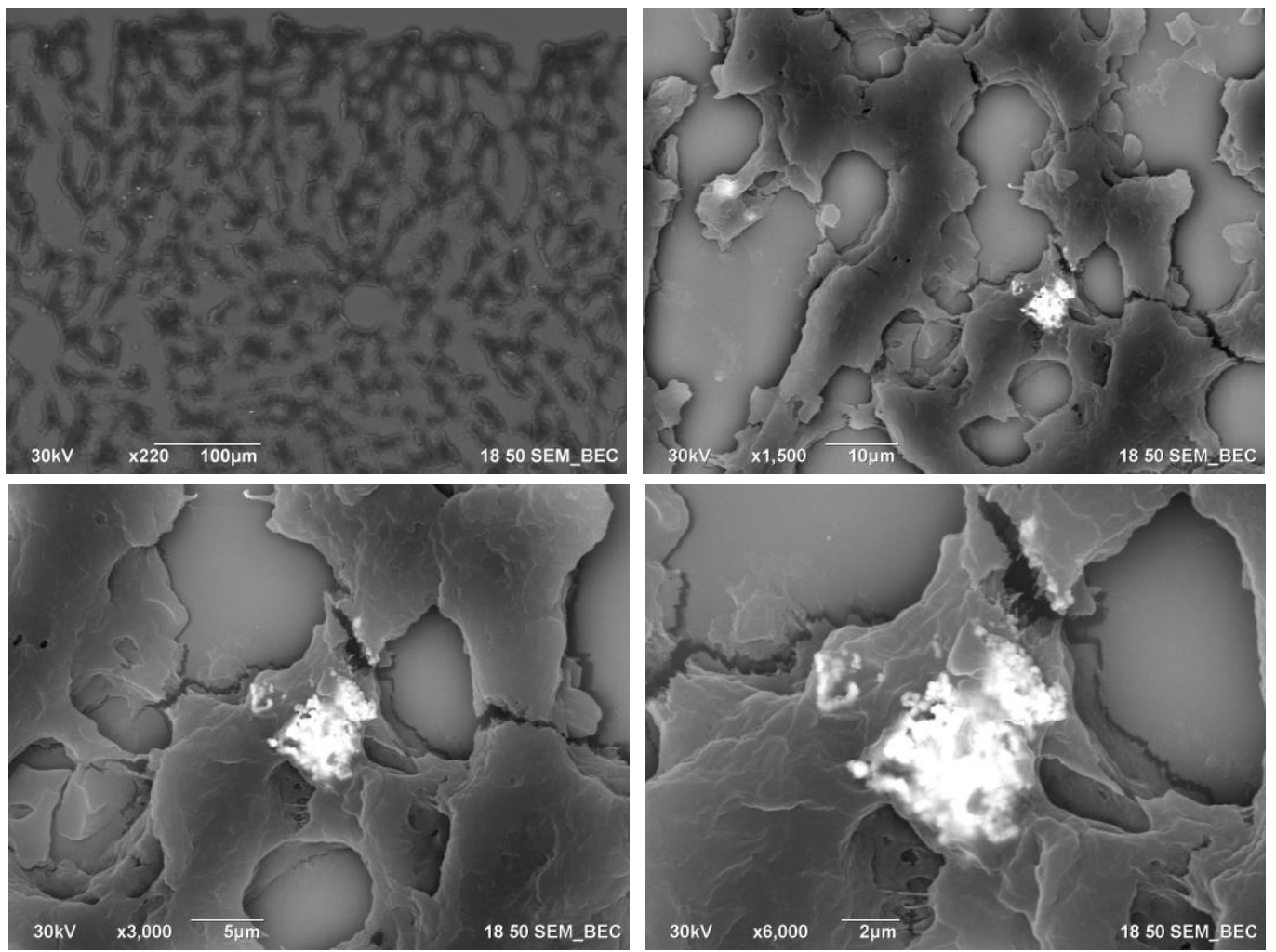

Figure 4.6. Particles in the liver

Different magnifications showing a particle cluster in the liver (100nm-1month post injection)

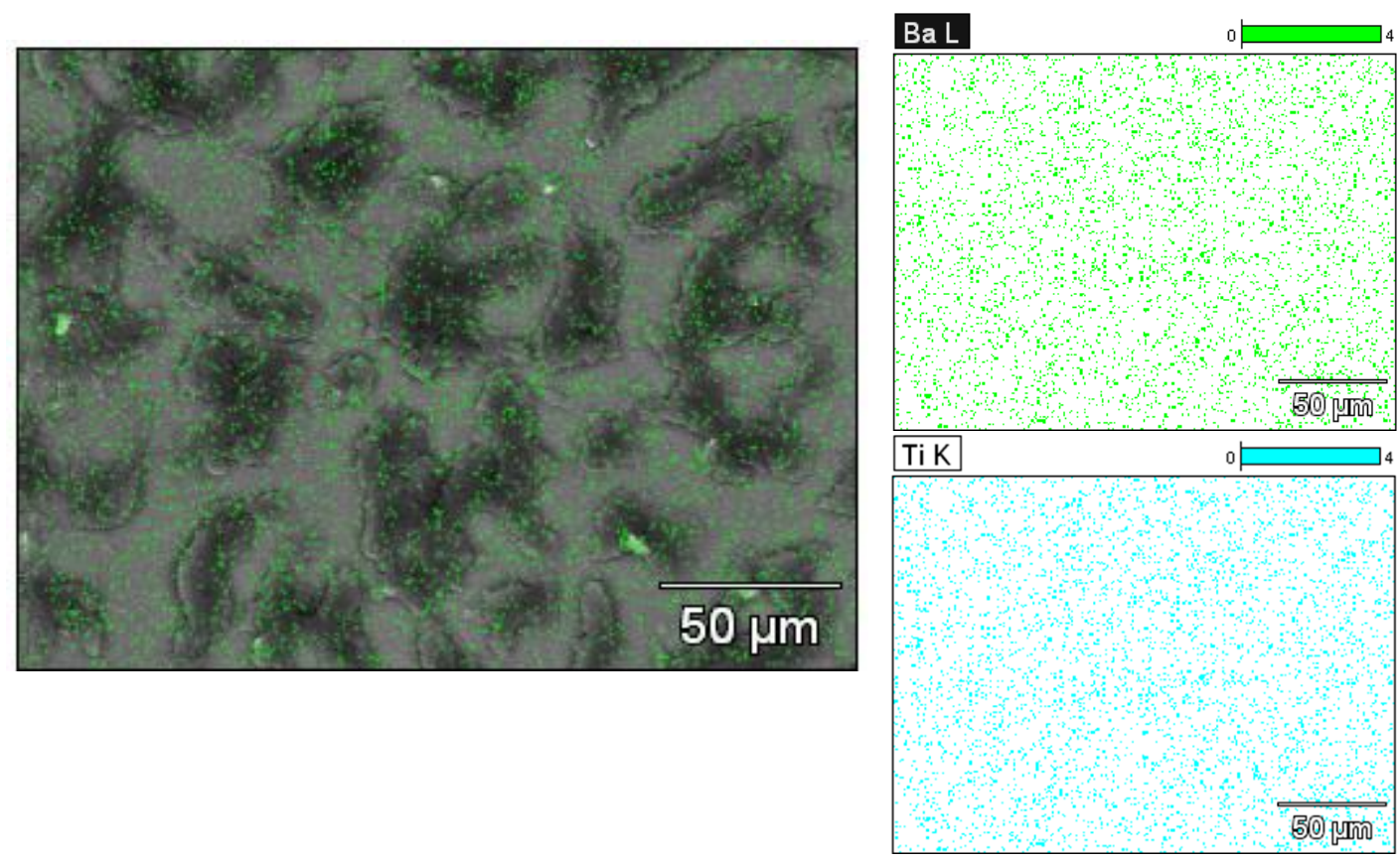

Figure 4.7. Mapping EDS of $100 \mathrm{~nm}$ MENs a month post injection in the liver 

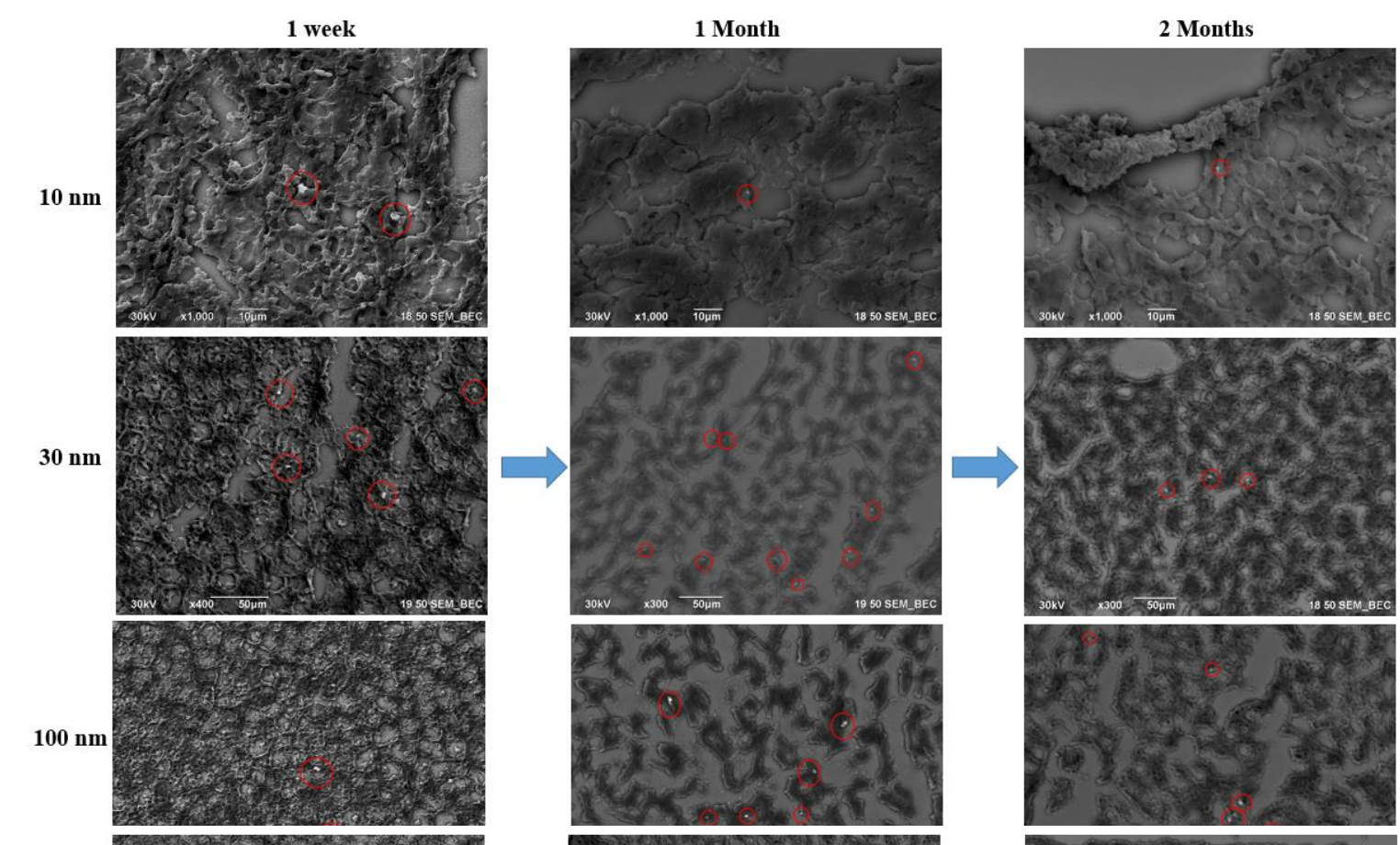

$600 \mathrm{~nm}$
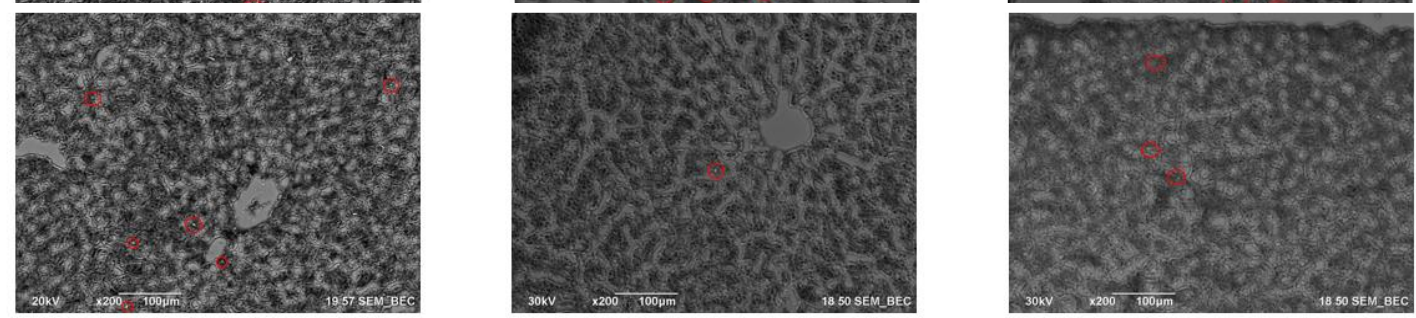

Figure 4.8. Liver schematic

Illustration of different MENs particles in the liver being discharged over time

1 week after the injection the samples seem to have an almost uniform distribution of particles everywhere in the liver. Additionally the particle distribution in the liver seems to be changing slower than in the other parts of the body. 
Table 4.2. The samples which were used for the quantitative analysis of the liver The table includes the number of the particles found by the software, the area that the particles cover, mean and standard deviation. In some samples only one area with particle agglomeration was found

\begin{tabular}{|c|c|c|c|c|c|}
\hline Time/ Size & Slice & count & $\%$ area & Mean $\%$ area & STDEV \\
\hline & 200x.bmp & 7 & 0.004 & & \\
\hline \multirow[t]{3}{*}{1 week $10 \mathrm{~nm}$} & 200x.bmp & 11 & 0.01 & 0.0057 & 0.0031 \\
\hline & $200 x-2 . b m p$ & 4 & 0.003 & & \\
\hline & $400 x-12 . b m p$ & 5 & 0.009 & & \\
\hline \multirow[t]{2}{*}{1 month $10 \mathrm{~nm}$} & $300 x-11 . b m p$ & 1 & 0.006 & 0.0070 & 0.0014 \\
\hline & $400 x-17 . b m p$ & 1 & 0.006 & & \\
\hline \multirow[t]{2}{*}{2 month $10 \mathrm{~nm}$} & 200x.bmp & 7 & 0.002 & 0.0020 & NA \\
\hline & 100x.bmp & 33 & 0.018 & & \\
\hline \multirow[t]{4}{*}{1 week $30 \mathrm{~nm}$} & $100 x-1 . b m p$ & 38 & 0.025 & 0.0183 & 0.0053 \\
\hline & $100 x-2 . b m p$ & 24 & 0.012 & & \\
\hline & 200x.bmp & 18 & 0.037 & & \\
\hline & $200 x-1 . b m p$ & 12 & 0.017 & & \\
\hline \multirow[t]{4}{*}{1 month $30 \mathrm{~nm}$} & 200x.bmp & 28 & 0.075 & 0.0666 & 0.0369 \\
\hline & $300 x-10 . b m p$ & 40 & 0.123 & & \\
\hline & $300 x-22 . b m p$ & 16 & 0.081 & & \\
\hline & 200x.bmp & 52 & 0.01 & & \\
\hline \multirow[t]{4}{*}{2 month 30nm } & 200x-1.bmp & 27 & 0.008 & 0.0070 & 0.0029 \\
\hline & 200x.bmp & 8 & 0.003 & & \\
\hline & 100x.bmp & 49 & 0.026 & & \\
\hline & 200x.bmp & 57 & 0.053 & & \\
\hline \multirow[t]{4}{*}{1 week $100 \mathrm{~nm}$} & 100x.bmp & 60 & 0.031 & 0.0363 & 0.0102 \\
\hline & 100x.bmp & 76 & 0.035 & & \\
\hline & $200 x-2 . b m p$ & 36 & 0.072 & & \\
\hline & $200 x-7 . b m p$ & 104 & 0.096 & & \\
\hline \multirow[t]{4}{*}{1 month $100 \mathrm{~nm}$} & $200 x-12 . b m p$ & 45 & 0.085 & 0.1016 & 0.0406 \\
\hline & $200 x-7 . b m p$ & 38 & 0.074 & & \\
\hline & $300 x \cdot b m p$ & 56 & 0.181 & & \\
\hline & $200 x-3 . b m p$ & 82 & 0.027 & & \\
\hline \multirow[t]{4}{*}{2 month $100 \mathrm{~nm}$} & $300 x \cdot b m p$ & 73 & 0.053 & 0.0313 & 0.0131 \\
\hline & $200 x-10 . b m p$ & 61 & 0.018 & & \\
\hline & 300x.bmp & 55 & 0.027 & & \\
\hline & $200 x-6 . b m p$ & 19 & 0.018 & & \\
\hline \multirow[t]{4}{*}{1 week $600 \mathrm{~nm}$} & $200 x-7 . b m p$ & 24 & 0.014 & 0.0320 & 0.0196 \\
\hline & $200 x-9 . b m p$ & 198 & 0.064 & & \\
\hline & $200 x-22 . b m p$ & 65 & 0.032 & & \\
\hline & 200x.bmp & 1 & 0.001 & & \\
\hline \multirow[t]{3}{*}{1 month $600 \mathrm{~nm}$} & 200x.bmp & 1 & 0.002 & 0.0013 & 0.0005 \\
\hline & $200 x-10 . b m p$ & 1 & 0.001 & & \\
\hline & $200 x-7 . b m p$ & 50 & 0.011 & & \\
\hline \multirow[t]{2}{*}{2 month $600 \mathrm{~nm}$} & 200x.bmp & 30 & 0.006 & 0.0133 & 0.0071 \\
\hline & $200 x-24 . b m p$ & 75 & 0.023 & & \\
\hline
\end{tabular}


Particle Distribution Over Time in Liver

0.1600

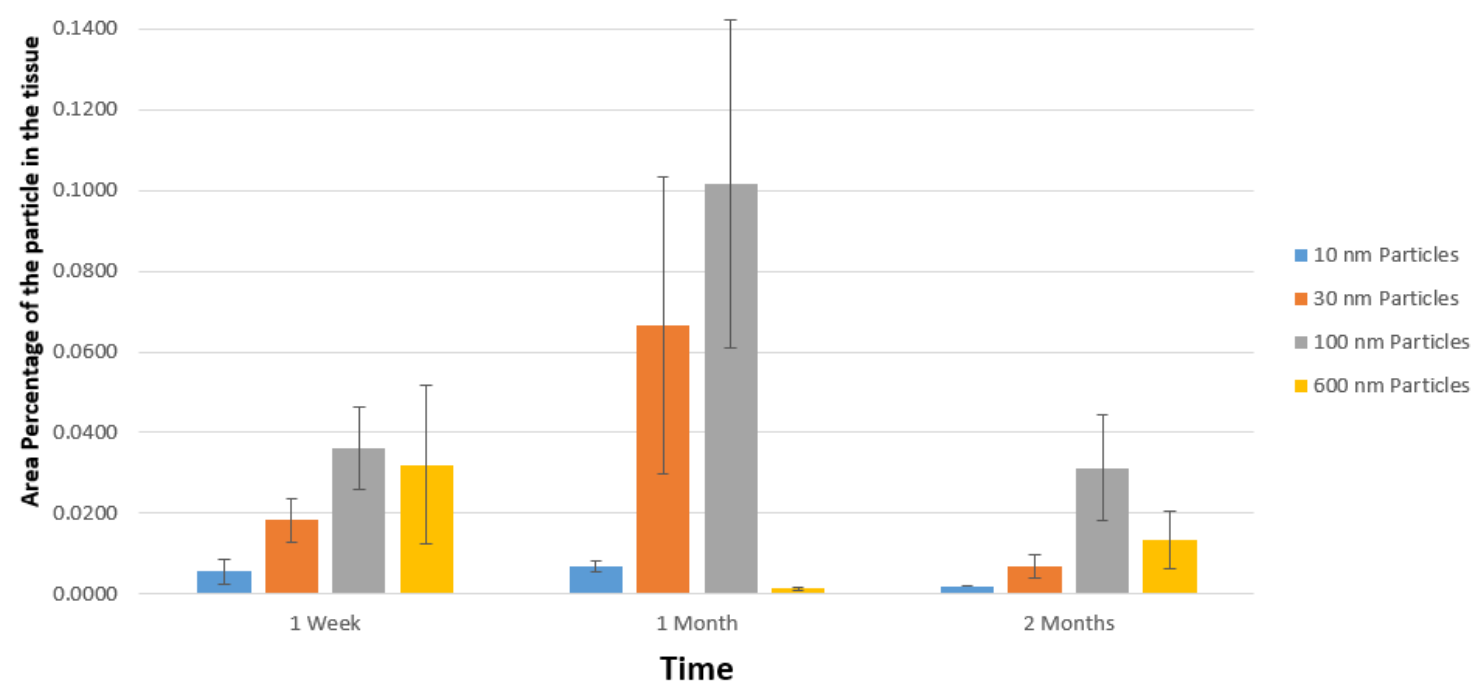

Figure 4.9. Liver distribution chart

Illustration of different size MENs particles in the liver being discharged over time

\section{PARTICLE DISTRIBUTION OVER TIME}

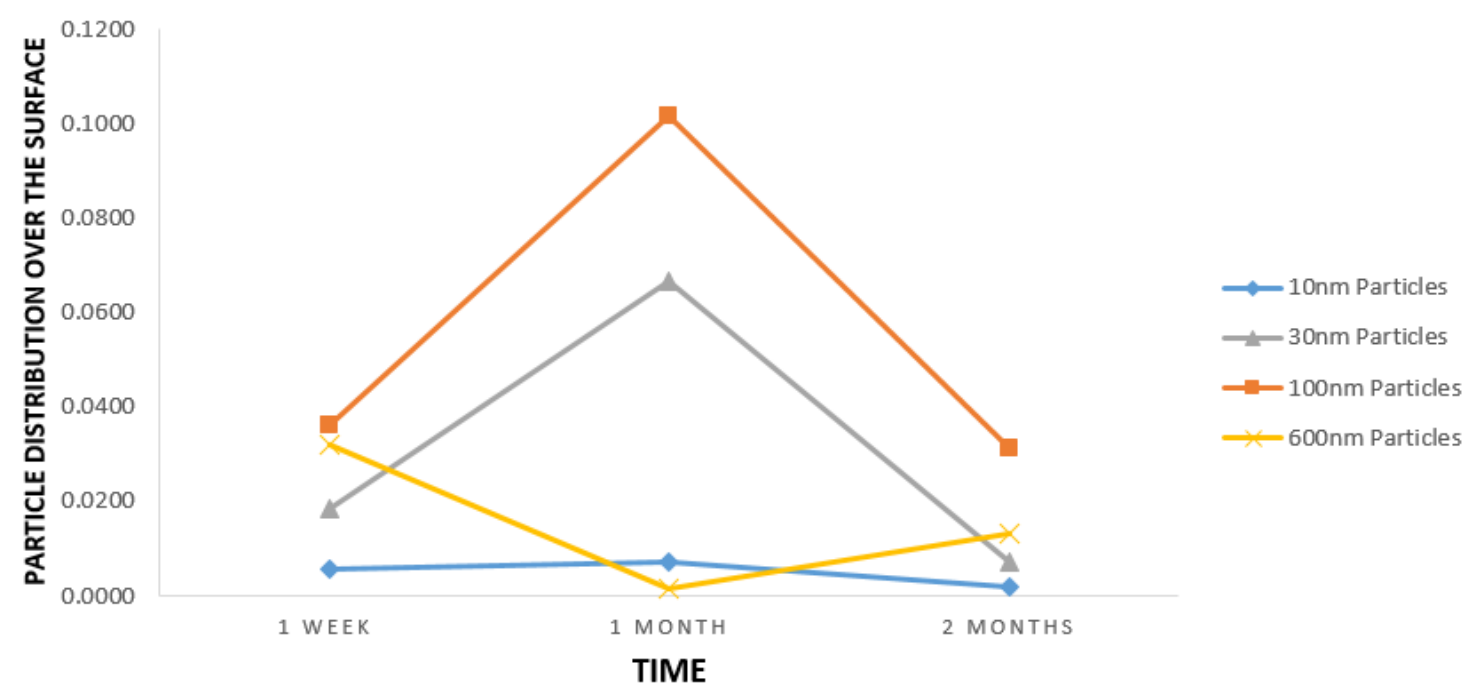

Figure 4.10. Liver distribution graph

Areal distribution of different sizes of MENs particles in the liver over time 


\section{Spleen}
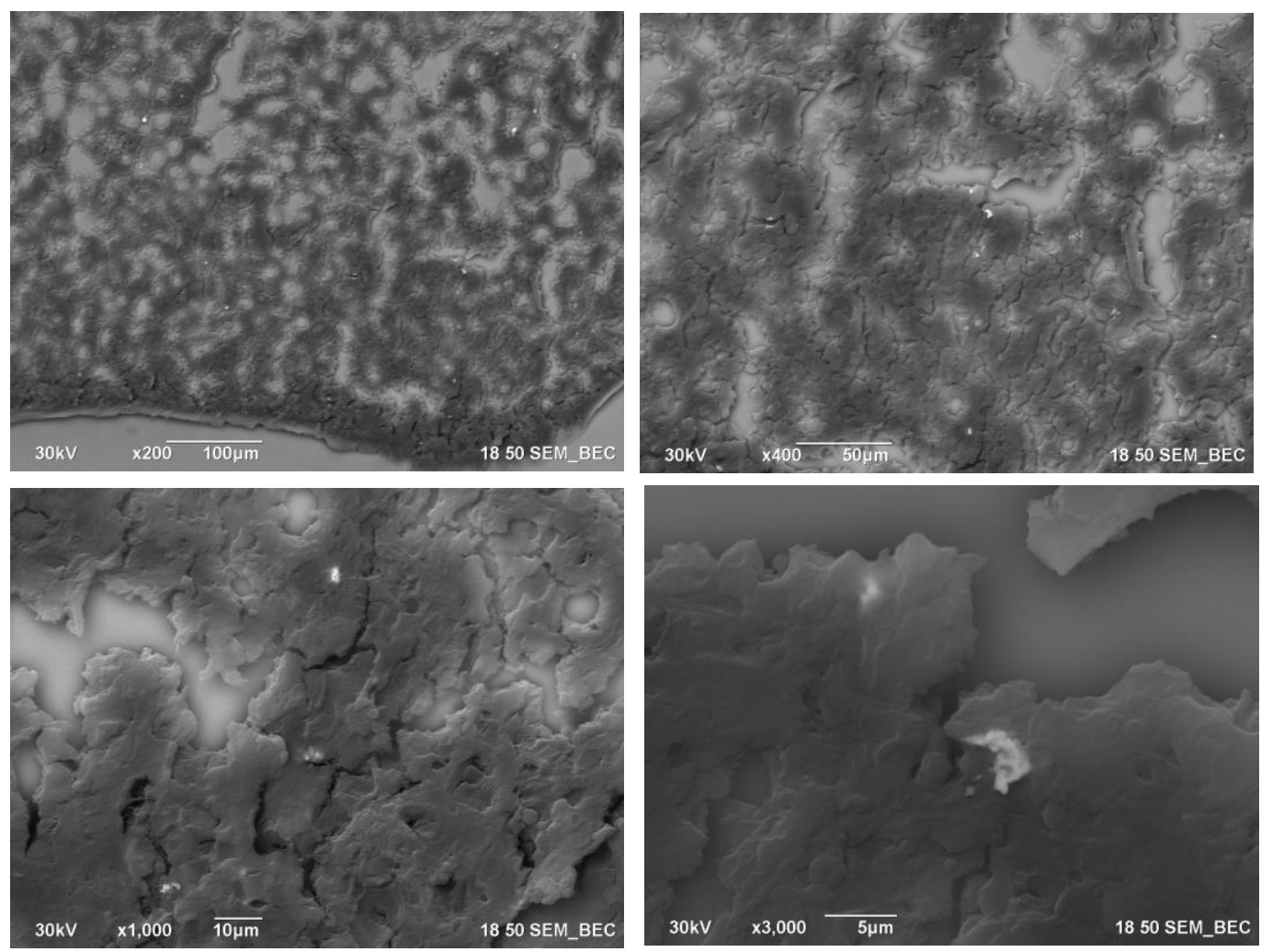

Figure 4.11. Particles in the spleen

Different magnifications showing a particle cluster in the spleen (100nm-1month post injection)

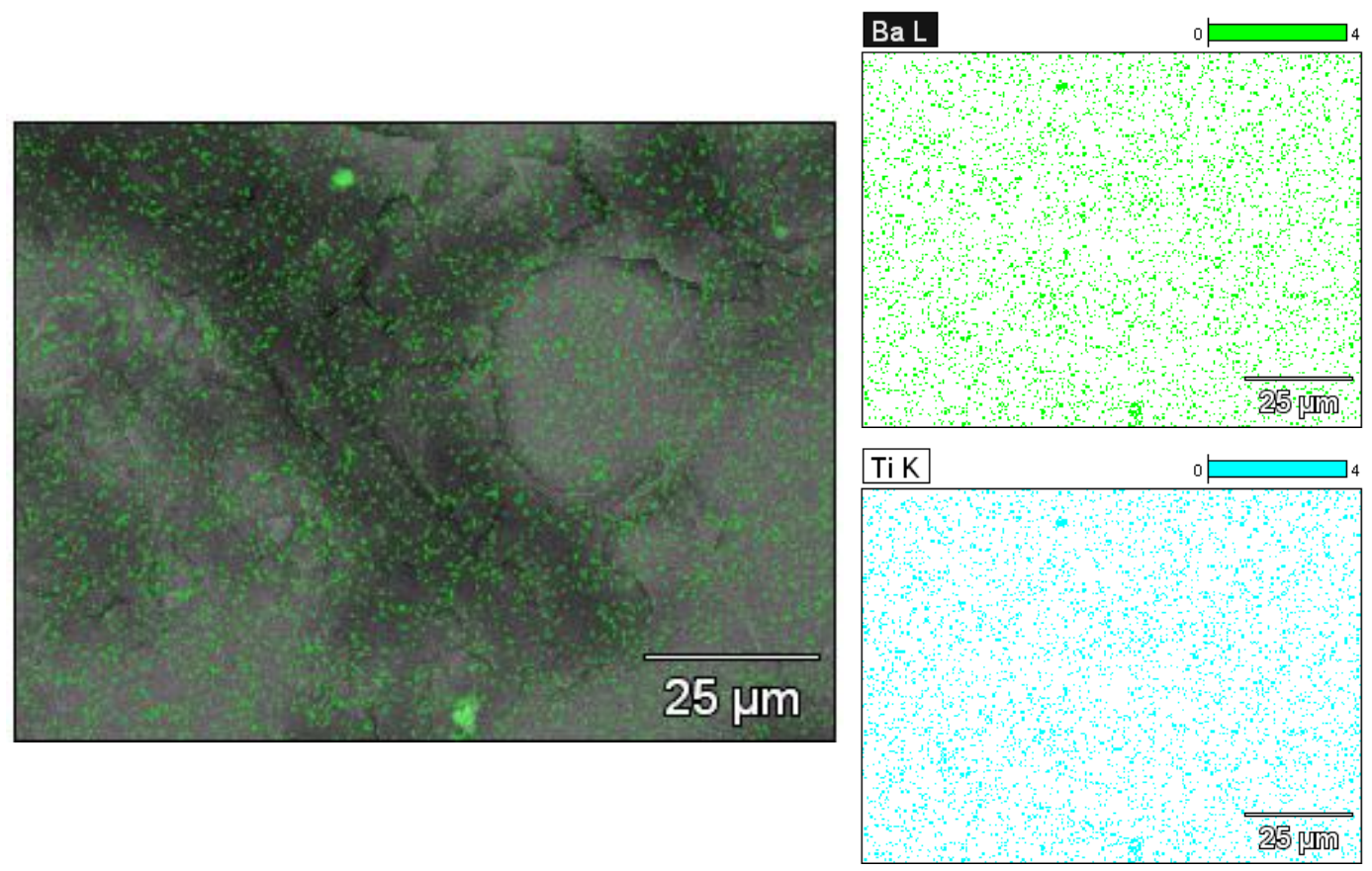

Figure 4.12. Mapping EDS of 100nm MENs a month post injection in the spleen 


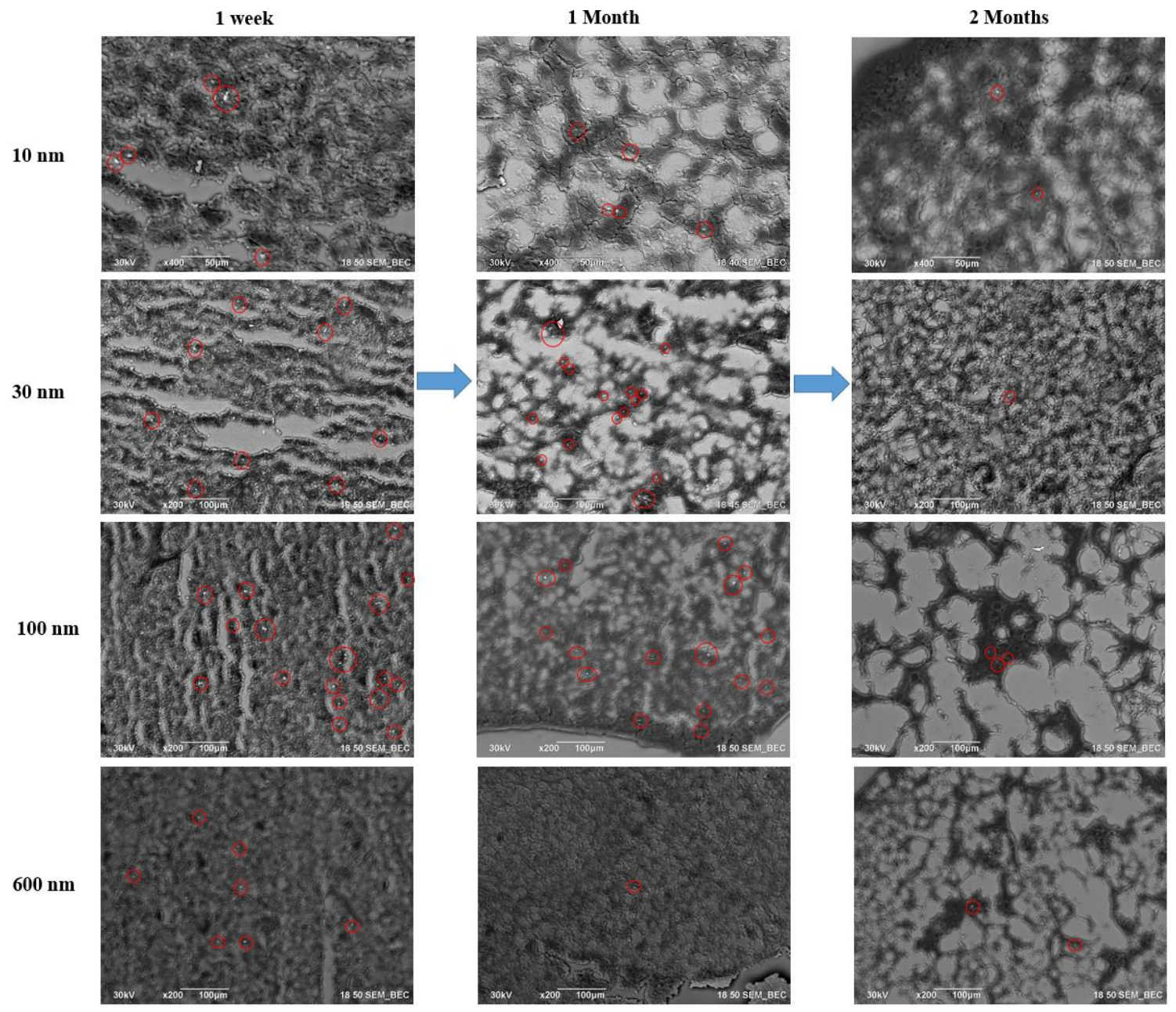

Figure 4.13. Spleen schematic

Illustration of different MENs particles in the spleen being discharged over time 
Table 4.3. The samples which were used for the quantitative analysis of the Spleen The table includes the number of the particles found by the software, the area that the particles cover, mean and standard deviation

\begin{tabular}{|c|c|c|c|c|c|}
\hline Time/ Size & Slice & count & $\%$ area & Mean \% area & STDEV \\
\hline & 200x-7.bmp & 204 & 0.048 & & \\
\hline \multirow[t]{3}{*}{1 week $10 \mathrm{~nm}$} & $300 x-5 . b m p$ & 154 & 0.021 & 0.0357 & 0.0111 \\
\hline & $200 x-4 . b m p$ & 190 & 0.038 & & \\
\hline & 200x.bmp & 15 & 0.006 & & \\
\hline \multirow[t]{3}{*}{1 month 10nm } & $200 x-7 . b m p$ & 157 & 0.029 & 0.0127 & 0.0116 \\
\hline & 200x-13.bmp & 13 & 0.003 & & \\
\hline & 200x.bmp & 2 & 0.001 & & \\
\hline \multirow[t]{5}{*}{2 month $10 \mathrm{~nm}$} & 200x.bmp & 1 & 0.001 & 0.0025 & 0.0021 \\
\hline & 200x.bmp & 3 & 0.002 & & \\
\hline & 200x.bmp & 18 & 0.006 & & \\
\hline & $200 x-5 . b m p$ & 191 & 0.04 & & \\
\hline & 200x.bmp & 218 & 0.043 & & \\
\hline \multirow[t]{4}{*}{1 week $30 \mathrm{~nm}$} & $200 x-6 . b m p$ & 241 & 0.059 & 0.0750 & 0.0373 \\
\hline & 300x.bmp & 325 & 0.139 & & \\
\hline & 200x.bmp & 229 & 0.094 & & \\
\hline & $200 x-3 . b m p$ & 252 & 0.03 & & \\
\hline \multirow[t]{3}{*}{1 month 30nm } & 200x.bmp & 76 & 0.021 & 0.0300 & 0.0073 \\
\hline & 200x.bmp & 122 & 0.039 & & \\
\hline & 200x.bmp & 1 & 0.001 & & \\
\hline \multirow[t]{3}{*}{2 month 30nm } & $200 x-1 . b m p$ & 6 & 0.002 & 0.0027 & 0.0017 \\
\hline & $200 x-2 . b m p$ & 11 & 0.005 & & \\
\hline & 200x.bmp & 462 & 0.118 & & \\
\hline \multirow[t]{3}{*}{1 week 100nm } & 200x.bmp & 403 & 0.12 & 0.1003 & 0.0264 \\
\hline & $200 x-6 . b m p$ & 221 & 0.063 & & \\
\hline & 200x.bmp & 112 & 0.035 & & \\
\hline \multirow[t]{3}{*}{1 month $100 \mathrm{~nm}$} & $200 x-4 . b m p$ & 54 & 0.026 & 0.0300 & 0.0037 \\
\hline & 200x-1.bmp & 58 & 0.029 & & \\
\hline & 200x.bmp & 28 & 0.053 & & \\
\hline \multirow[t]{3}{*}{2 month $100 \mathrm{~nm}$} & 200x.bmp & 4 & 0.003 & 0.0227 & 0.0218 \\
\hline & $200 x-1 . b m p$ & 19 & 0.012 & & \\
\hline & $200 x-2 . b m p$ & 234 & 0.066 & & \\
\hline \multirow[t]{3}{*}{1 week $600 \mathrm{~nm}$} & $200 x-3 . b m p$ & 132 & 0.035 & 0.0447 & 0.0151 \\
\hline & $200 x-4 . b m p$ & 105 & 0.033 & & \\
\hline & $200 x-1 . b m p$ & 16 & 0.003 & & \\
\hline \multirow[t]{3}{*}{1 month $600 \mathrm{~nm}$} & $200 x-4 . b m p$ & 15 & 0.006 & 0.0050 & 0.0014 \\
\hline & $200 x-5 . b m p$ & 20 & 0.006 & & \\
\hline & 200x.bmp & 42 & 0.01 & & \\
\hline \multirow[t]{2}{*}{2 month $600 \mathrm{~nm}$} & 200x.bmp & 5 & 0.002 & 0.0073 & 0.0038 \\
\hline & 200x.bmp & 47 & 0.01 & & \\
\hline
\end{tabular}


Particle Distribution Over Time in Spleen

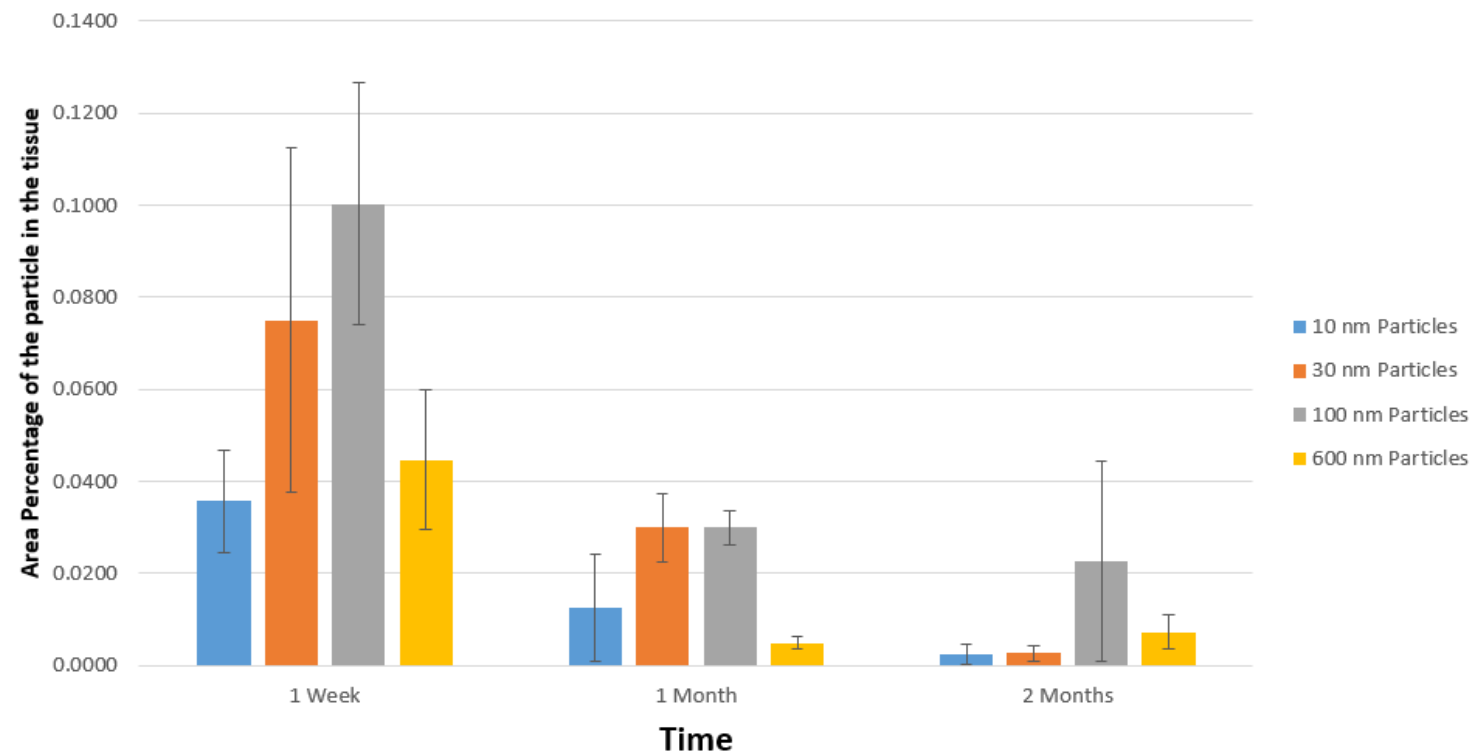

Figure 4.14. Spleen distribution chart

Illustration of different size MENs particles in the spleen being discharged over time

PARTICLE DISTRIBUTION OVER TIME

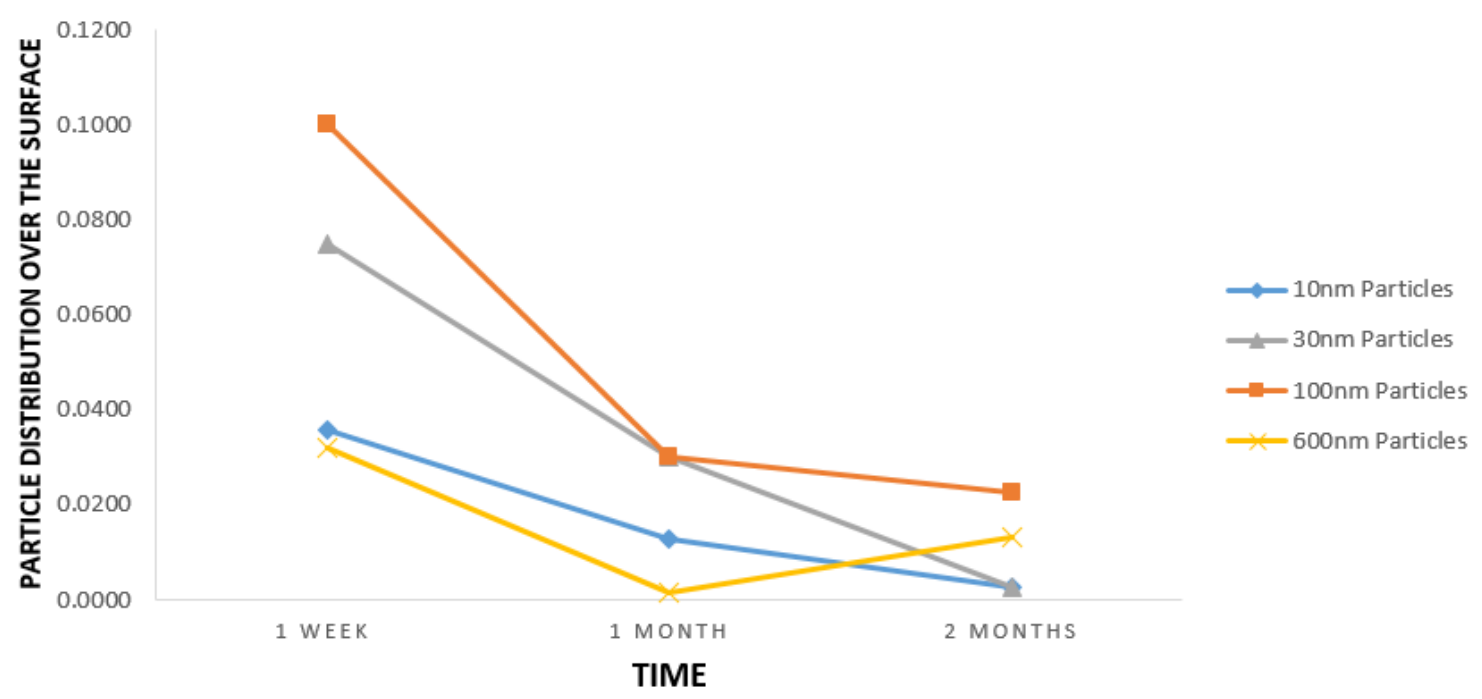

Figure 4.15. Spleen distribution graph

Areal distribution of different sizes of MENs particles in the spleen over time 


\section{Kidney}
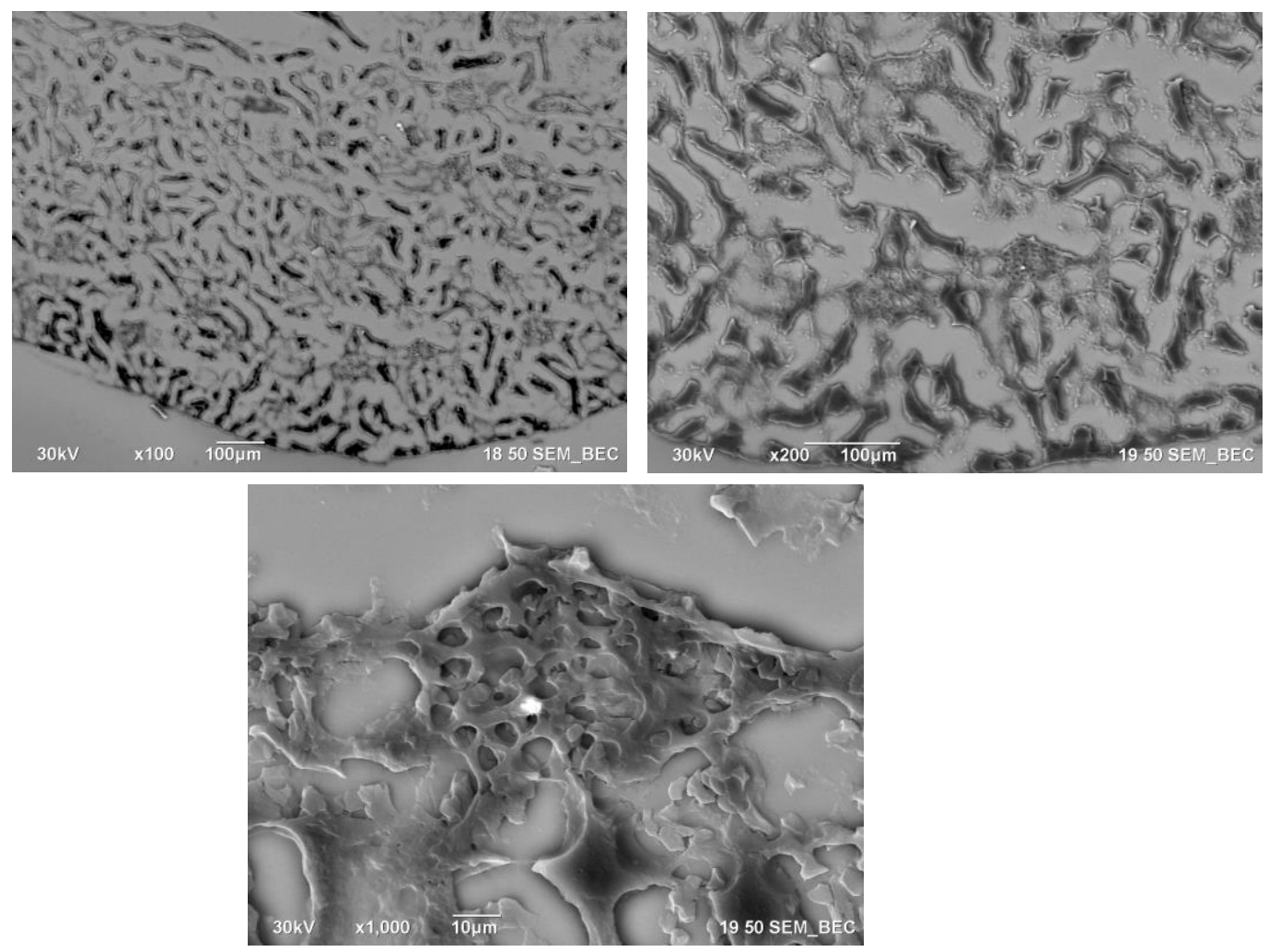

Figure 4.16. SEM of the kidney

Different magnifications showing a particle cluster in the kideny (100nm-1 week post injection)
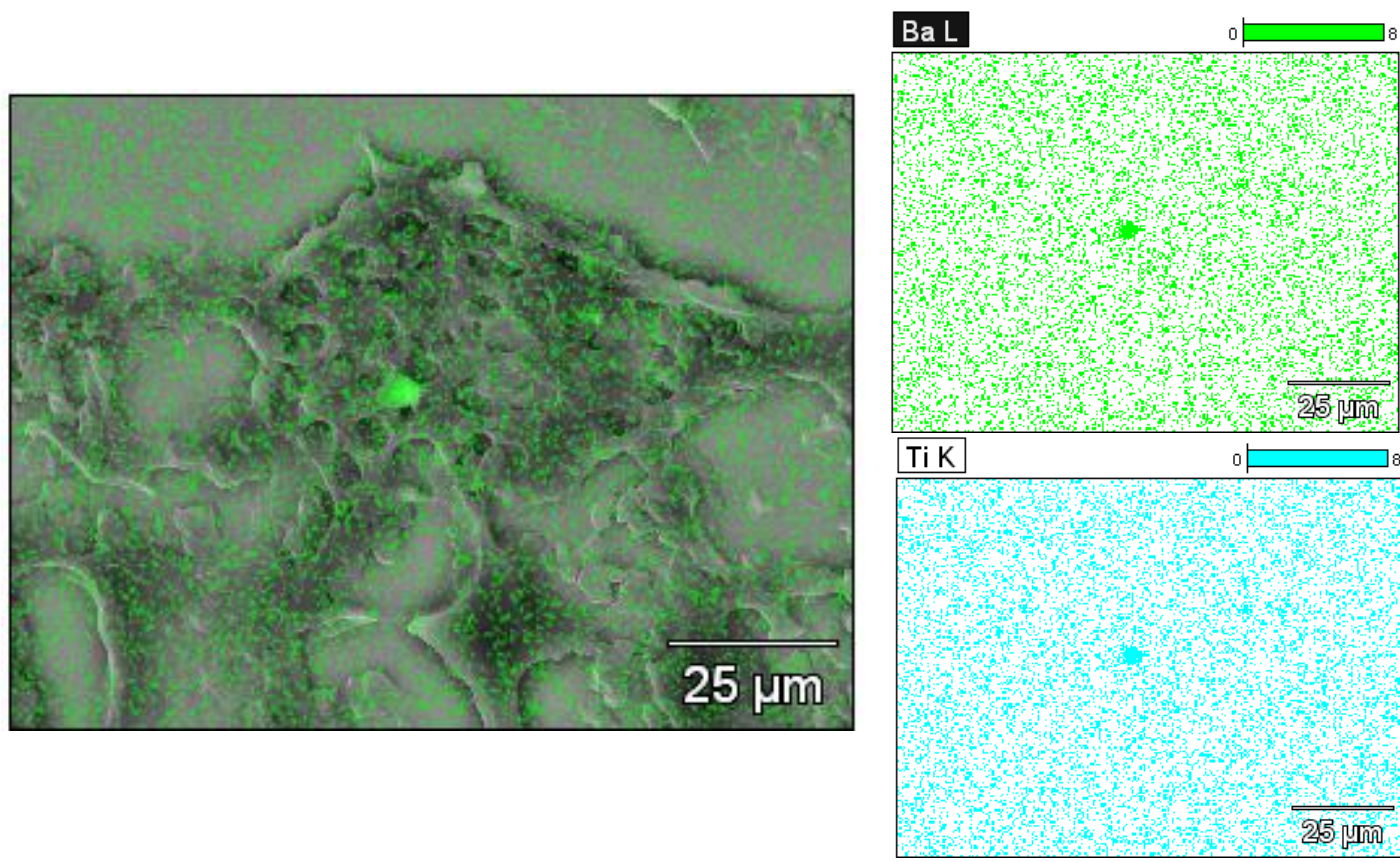

Figure 4.17. Mapping EDS of $100 \mathrm{~nm}$ MENs a week post injection in the kidney 


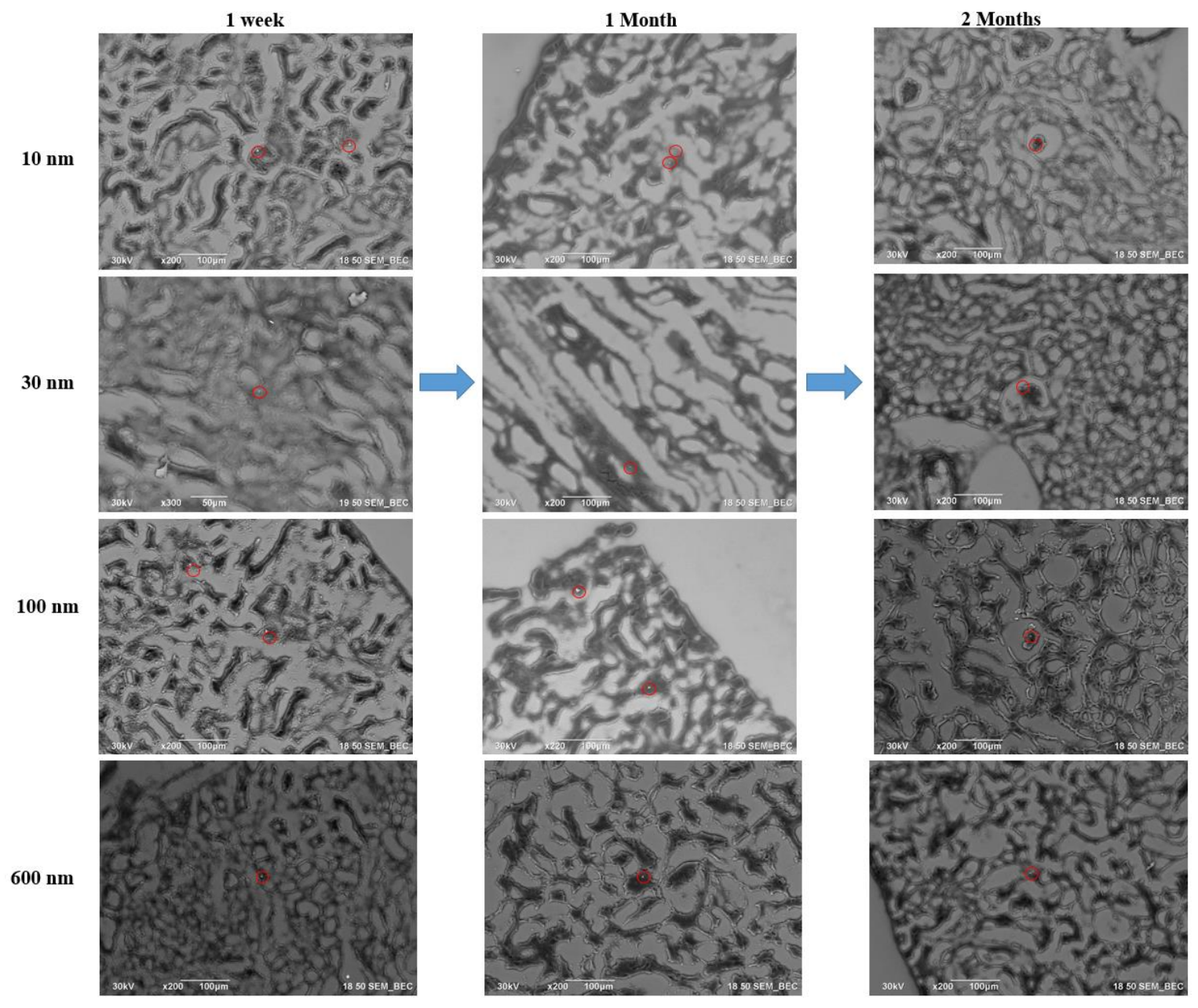

Figure 4.18. Kidney schematic

Illustration of different MENs particles in the kidney being discharged over time

Due to the sparsity of particle clusters in the kidney, a quantitative analysis of particles in the organ that yields meaningful data has proved to be difficult. The kidney consists of different anatomical components. One of the important parts of the kidney is the glomerulus, which is a cluster of capillaries around the end of a kidney tubule where waste products are filtered from the blood. This part of kidney, along with some other parts, is responsible for filtering the blood. A study of the kidney has confirmed that the kidney has 
three different layers in glomerular capillary wall. These layers are the endothelium, the basement membrane, and the visceral epithelium [2].

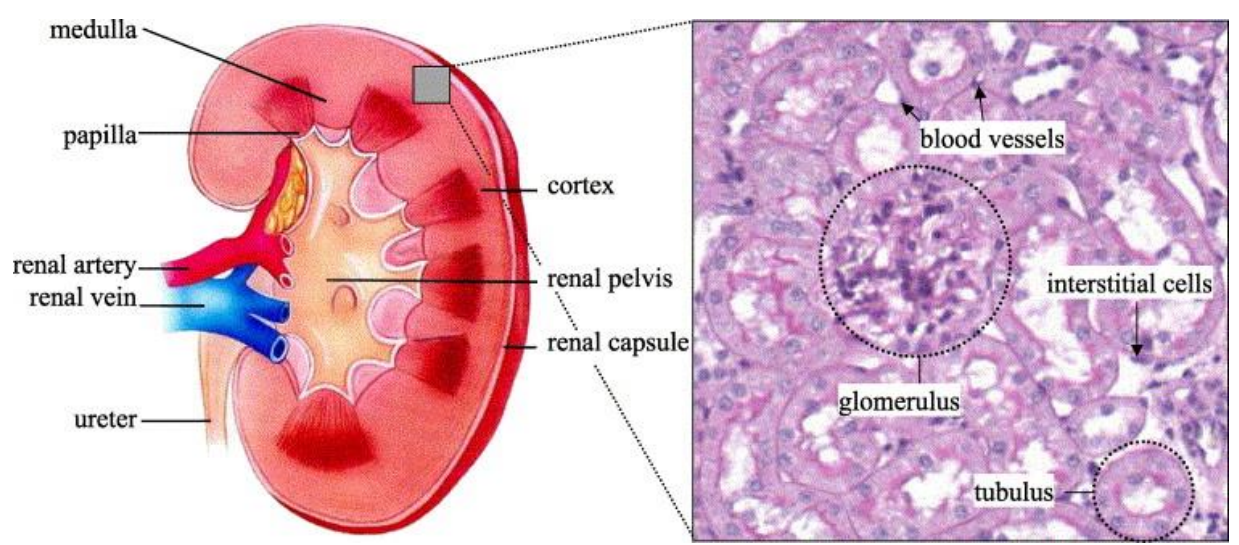

Fig 4.19. Relative anatomic location of various cell types in the kidney. Schematic picture of a kidney and a microscopic image of the renal cortex [1]

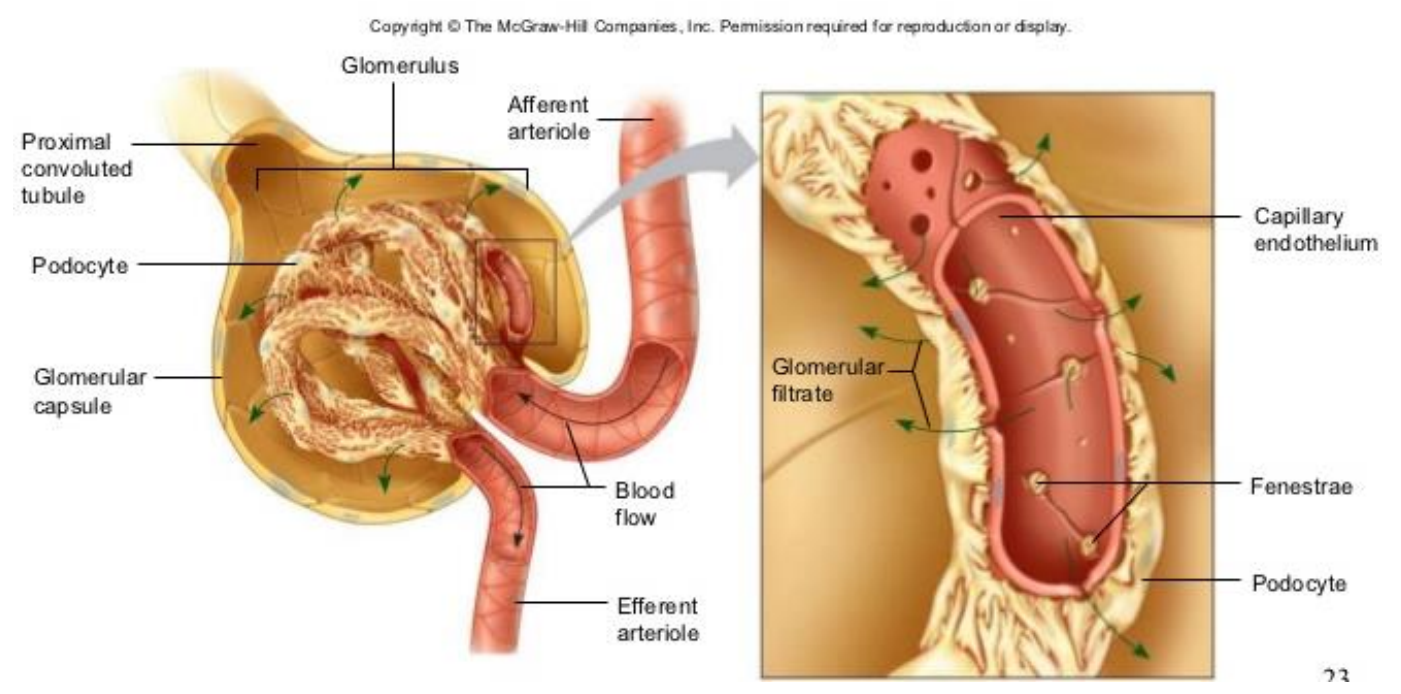

Figure 4.20. Schematic picture of the glomerular capillary [1]

It is assumed that in order to be able to facilitate diffusion, the glomerular capillary is highly specialized. It has epithelial cells that are highly branched and have numerous terminal processes that interdigitate with one another. Due to the existence of significant diffusion spaces between the processes, this layer cannot behave as a physiological barrier unless epithelial cells are contractile. The thin sheet of endothelial cytoplasm is perforated by a 
closely spaced system of holes. These pores average a little over 0.1 microns in diameter and this allows an unimpeded passageway for even the largest molecules. The endothelium is perforated by holes just like the glomerular endothelium. However, these pores are smaller (about $0.6 \mu$ ). These pores are also spaced more widely. In the medulla, only patches of fenestrated endothelium are found [3]. Basically, the glomerulus is filled with little pores and it has especially adapted endothelial cells which are fenestrated. These cells provide a thin selective membrane. Fenestrations in these cells are large enough for all the particles in the blood to pass through short of whole cells, but the pores are negatively charged and the other side of the cell is covered with smaller slit pores. This collection of cells only let the water and plasma through, as proteins and cells cannot pass.

This study has found that as the particles first enter the kidney they can be found in different parts of the organ, but as the time passes the particles get discharged and the only part that traps and accommodates clusters of the particles are the glomeruli. The glomeruli are also acting as pressure valves for keeping the blood flow constant by changing their size. The figures that follow show the images taken of these glomeruli at different times after injection. It seems that the glomeruli which had the particles for longer periods of time have grown smaller, which might be due to the increased pressure which particles in the glomerulus have caused. 

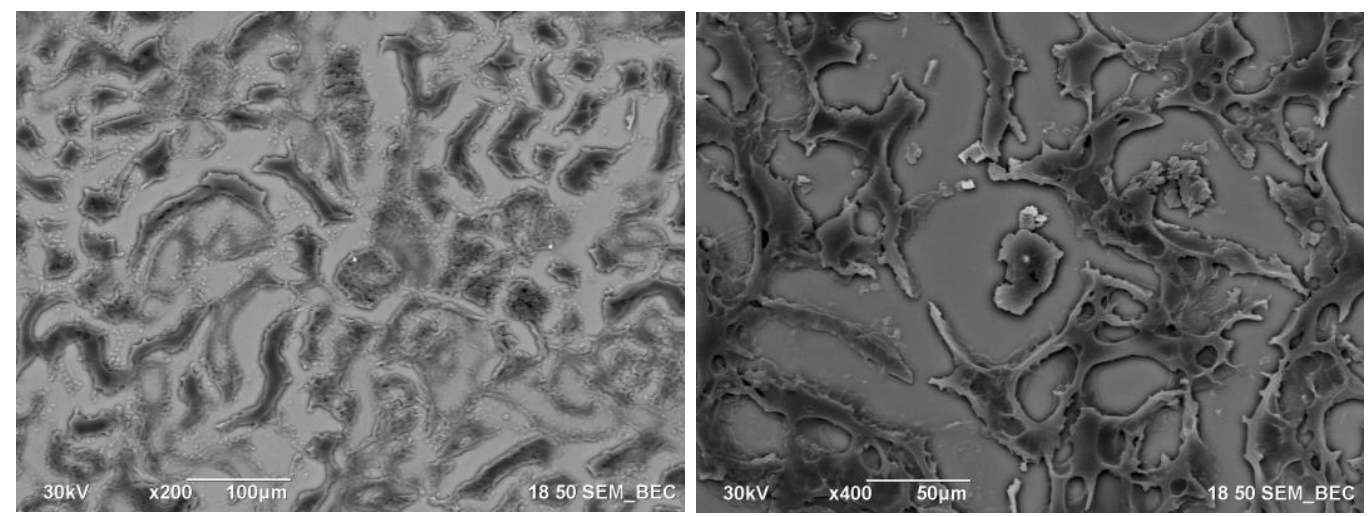

Figure 4.21. SEM of particles in the glomerular capillary

Left picture shows a cluster of $10 \mathrm{~nm}$ particles in the glomerulus after a week while the right image shows the glomerulus with $100 \mathrm{~nm}$ particles after 2 months

\section{Brain}

Particle clusters in the brain were rare and small in both size and quantity. Hence, making a schematic such as the ones done for the other oragns (i.e., liver, lung and spleen) relatively complicated. It made more sense to show the clusters which were found in the brain with the illustration which follow. These clusters are usually small ( 2 microns). Some of clusters of the particles in the brain were found in the cortex. Since, the bodies of cells which can become malignant (astrocytes mostly) are located in the cortex, and are at the origin of the most aggressive tumors in the brain (astrocytoma, glioblastoma multiform), these particles seem to have an advantage for being used as drug carriers for the brain tumors.
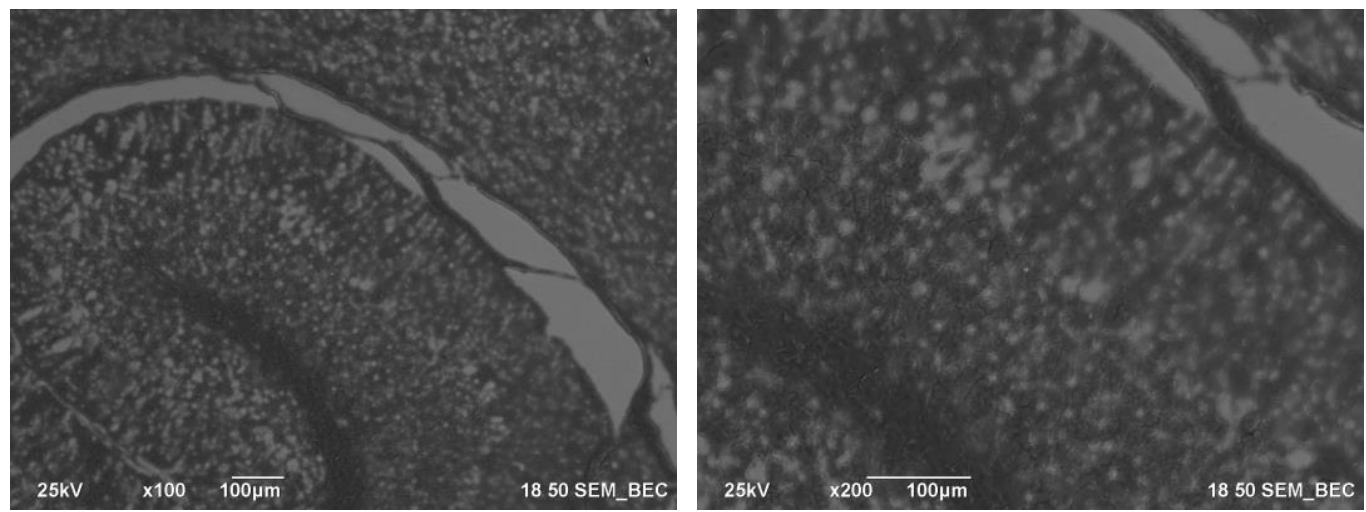

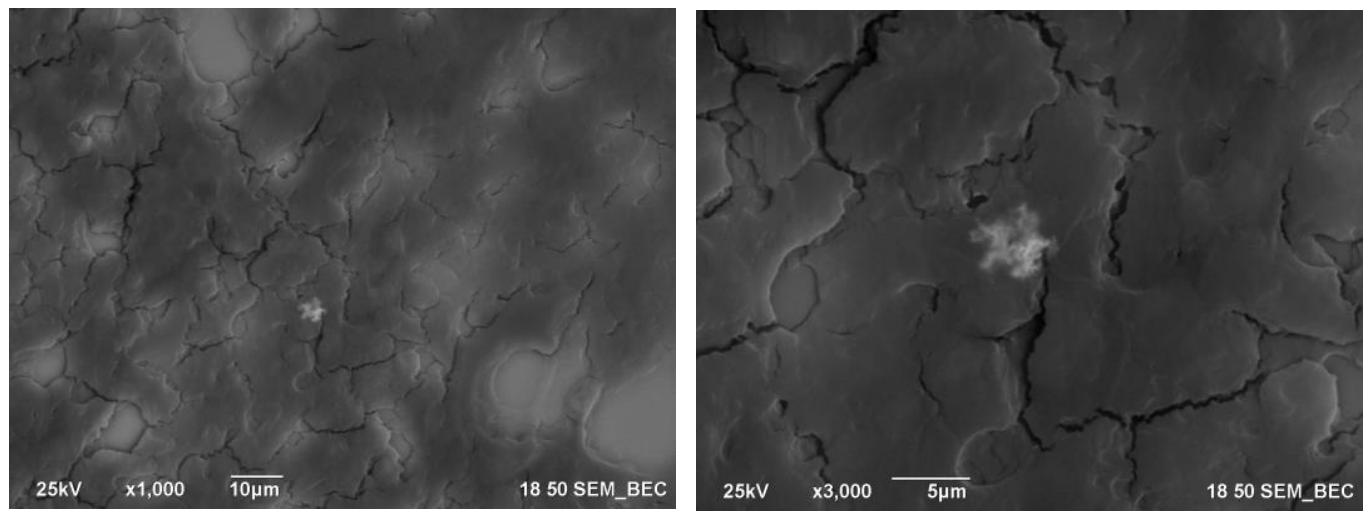

Figure 4.22. SEM image of a section of the brain (cerebellum)

Different magnifications showing a particle cluster in the brain (600nm-1 week post injection)

\section{Week Post Injection}

\section{Month Post Injection}

\section{Months Post Injection}
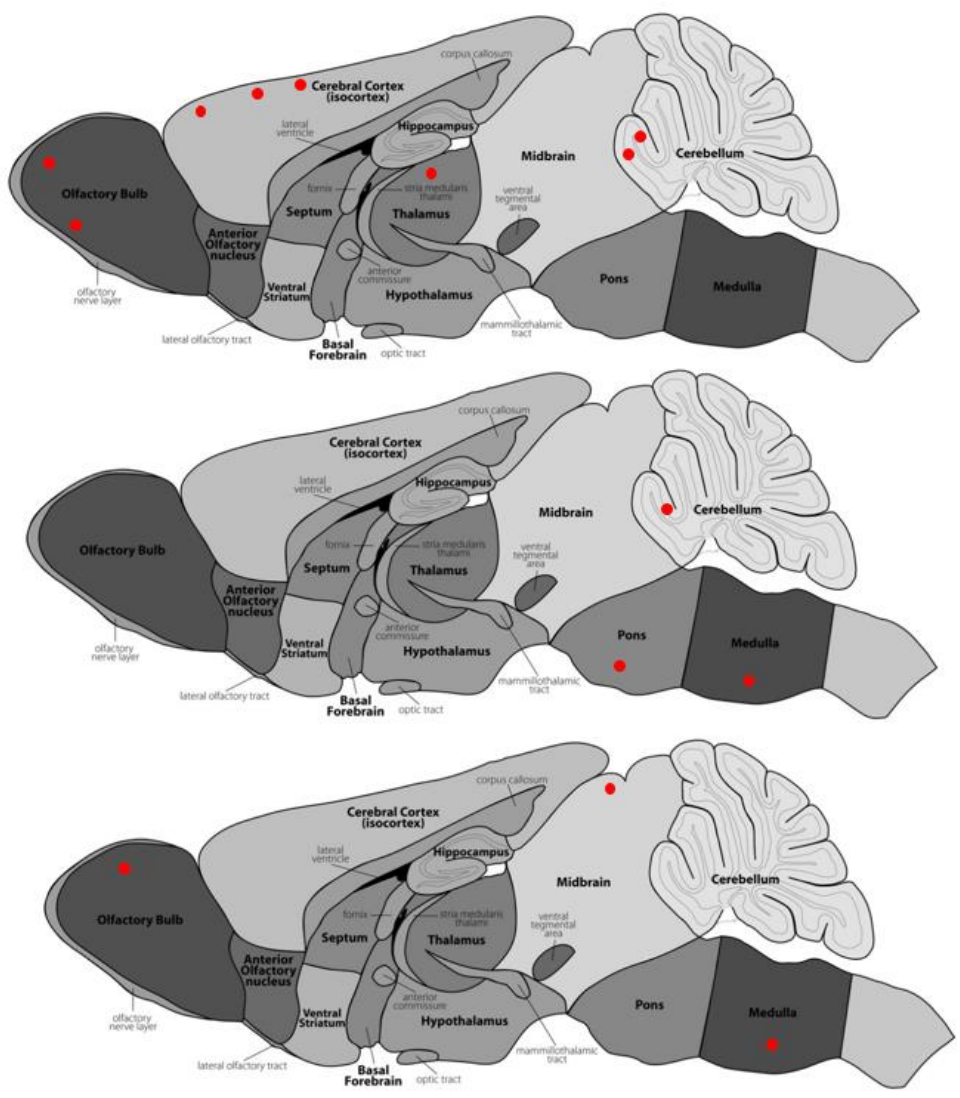

Figure 4.23. Brain schematic

$600 \mathrm{~nm}$ particles in brain over time 


\section{Total distribution and the effect of size}

Since the graphs and data for the distribution of particles in each organ is available, the distribution of particles in the whole body can be mapped based on the data gathered in the previous steps. It should be noted that since the amount of particles in the brain and in the kidney was relatively low, they were not taken into the account for this part of the study.

\section{Distribution of $10 \mathrm{~nm}$ Particles over time}

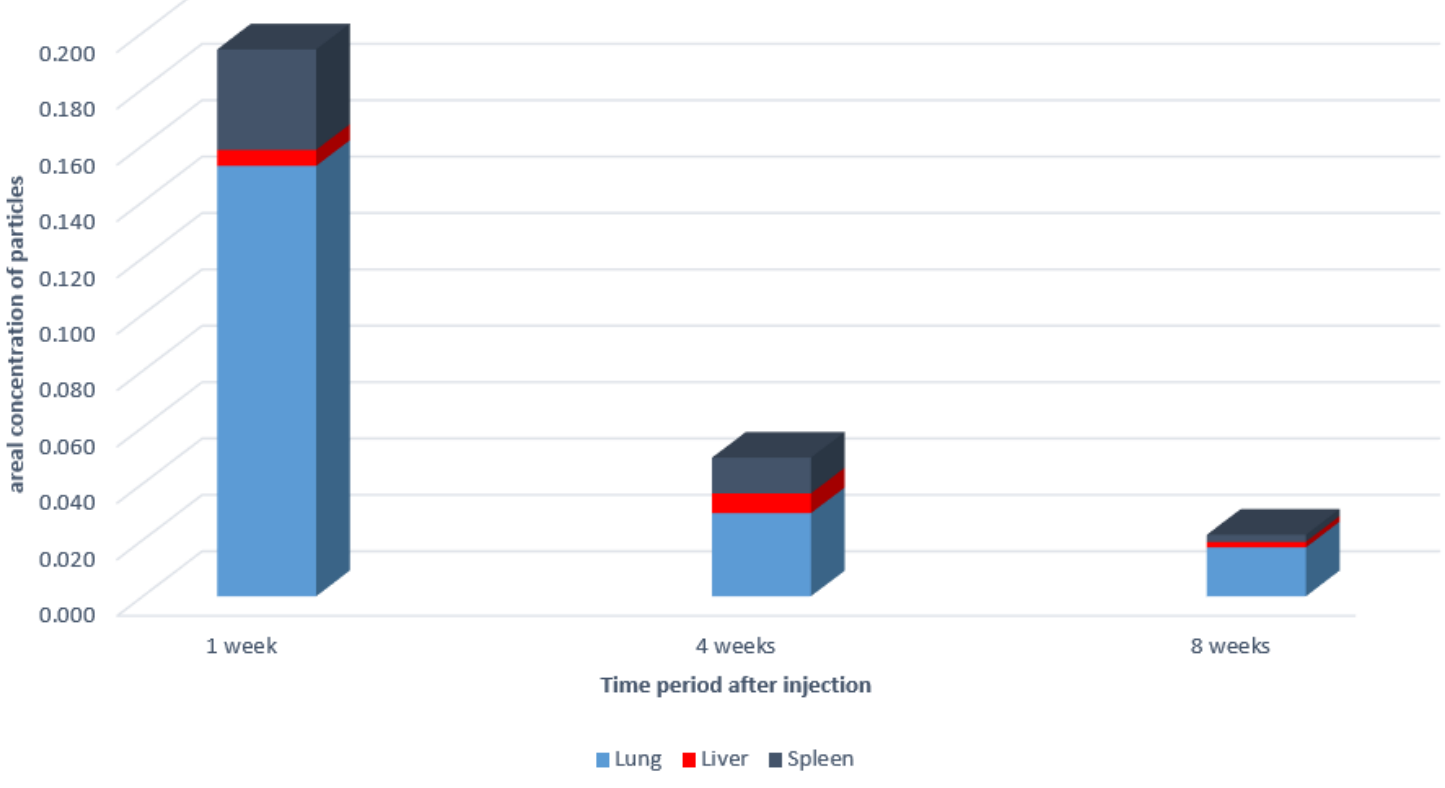

Figure 4.24. Bio-distribution of $10 \mathrm{~nm}$ particles over time 
Distribution of $\mathbf{3 0 ~} \mathrm{nm}$ Particles over time

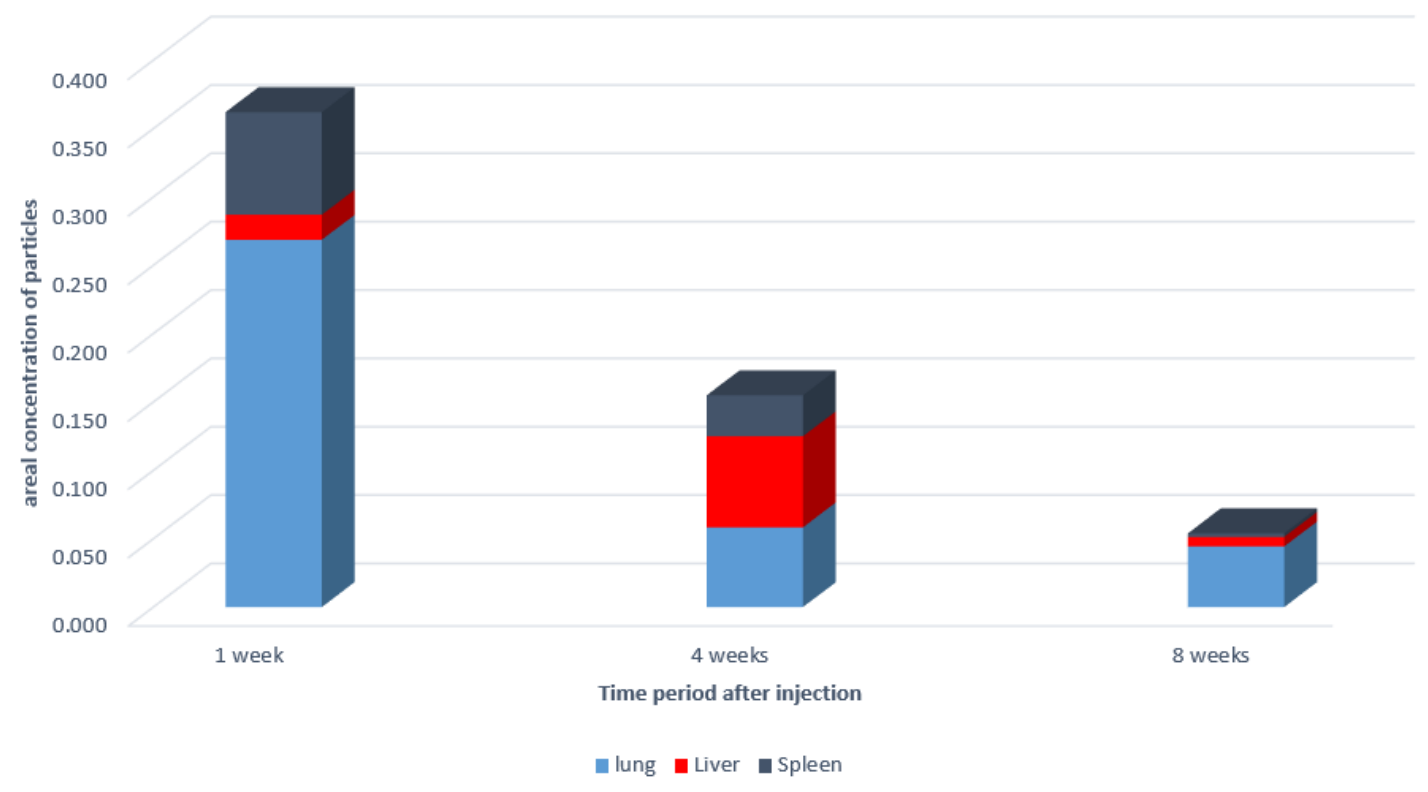

Figure 4.25. Bio-distribution of $30 \mathrm{~nm}$ particles over time

\section{Distribution of $100 \mathrm{~nm}$ Particles over time}

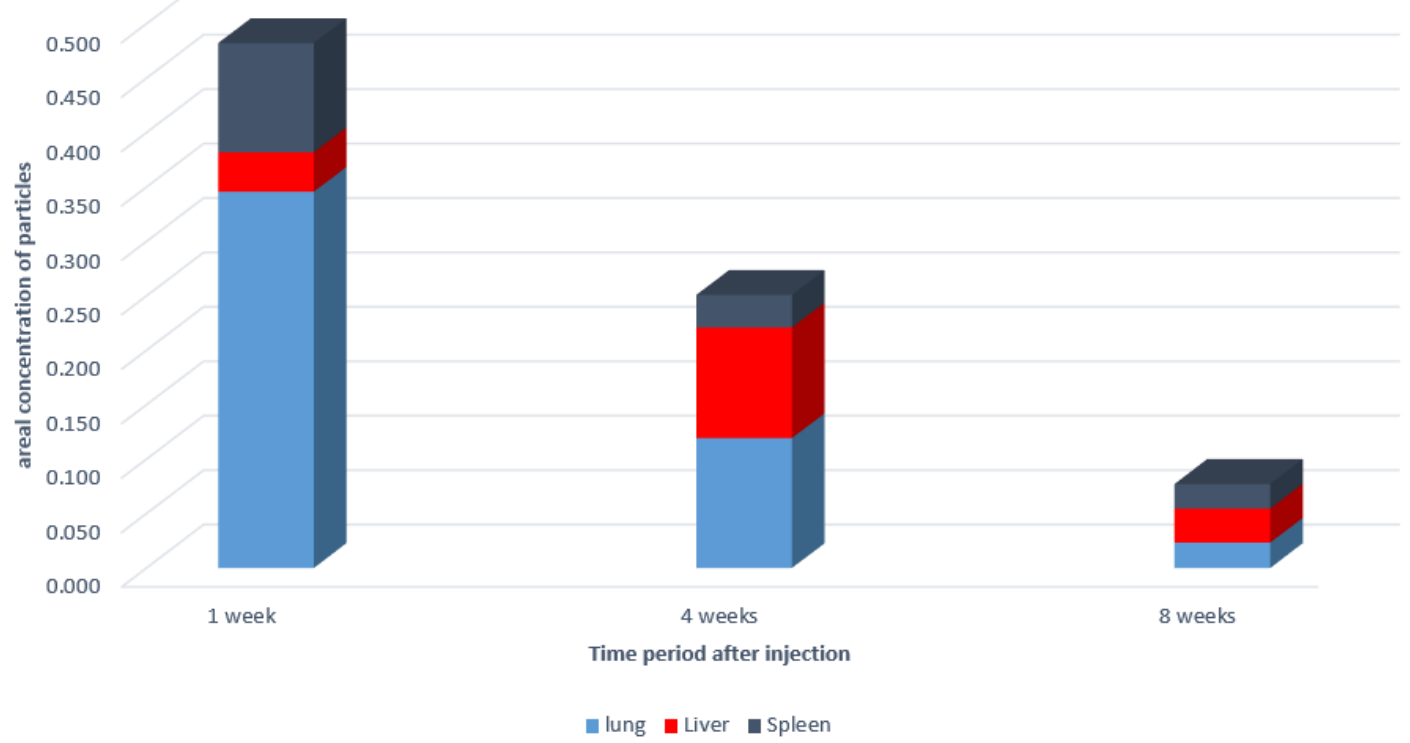

Figure 4.26. Bio-distribution of $100 \mathrm{~nm}$ particles over time 


\section{Distribution of $600 \mathrm{~nm}$ Particles over time}

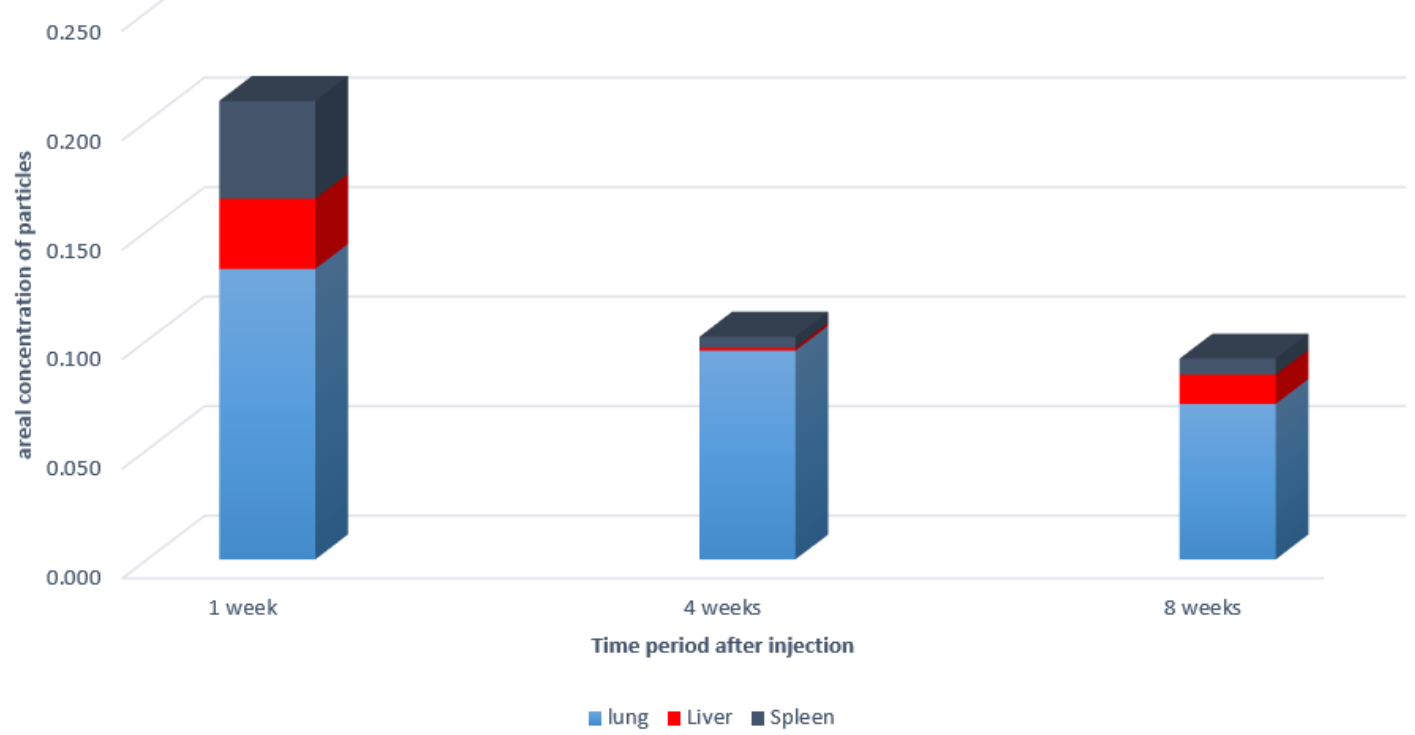

Figure 4.27. Bio-distribution of $600 \mathrm{~nm}$ particles over time

The amount of particles injected into each mouse was equal and close to $5 \mathrm{mg}(<10 \%$ was lost during the injection). It is clear that one of the key parameters that plays a role in how long the particles stay in the body is the size of the nanoparticles. This parameter is important due to the fact that it affects factors such as hepatic filtration, tissue extravasation, tissue diffusion, and kidney excretion. The higher amount of particles detected after 1 week for particles larger than $10 \mathrm{~nm}$ and smaller than $500 \mathrm{~nm}$ can be explained as follows. Very small particles $(<10 \mathrm{~nm})$ have a high tendency to leave the body through kidney filtration in large quantities. As the particles grow larger, the rate of their discharge through the kidney slows down. However, particles larger than 100nm have a higher tendency to absorb proteins onto their surface, which leads to opsonization and eventually hepatic uptake rates that are larger than those for smaller sized particles. Smaller sized particles cluster less, and might also form smaller clusters which cannot be 
detected by the microscope. Since their clusters are smaller, they take less time to get discharged out of the body. This explains why $600 \mathrm{~nm}$ particles seem to be an exception to the observed trend. These particles seem to be detected less than expected at each stage of the study. This leads to the conclusion that they are being discharged by a mechanism different from the discharge mechanism of the other particles (e.g. improved hepatic uptake). This observation has also been confirmed by a study of a different category of nanoparticles by Alexis et al. Their observations of nanoparticles that ranged between 50 and $500 \mathrm{~nm}$ shows that higher levels of agglomeration of the larger nanoparticles in the liver. They propose that the mechanism of hepatic uptake was mediated by surface absorption of proteins leading to opsonization [4].

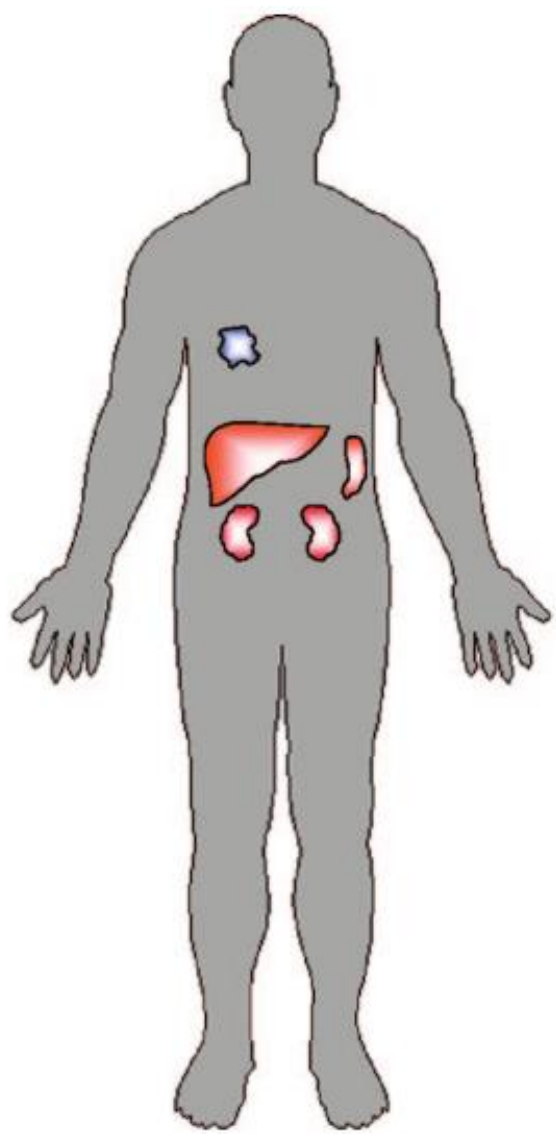

\section{Liver and Spleen}

- Clearance by phagocytic uptake and hepatic filtration

- Improve circulation half-life through particle sizes $\leq 100 \mathrm{~nm}$ and negative or neutral surface charge

\section{Kidneys}

- Clearance through excretion

- Improve circulation half-life through particle sizes $\geq 10 \mathrm{~nm}$

Figure 4.28. Biodistribution and clearance of nanoparticles.

Tissue defects, stealth properties, targeting, and the size of the nanoparticles are major factors affecting the biodistribution and clearance of nanoparticles[4]. 


\section{Nanoelectronics}

Before the fibbing process, a TEM sample was extracted from a pristine MTJ sample and was put on a TEM grid using FIB and in-situ lift out processes to confirm that the MTJ layer was deposited onto the wafer. Additionally, in order to confirm the properties of the deposited junction before FIB trimming, pristine sputter-deposited ferromagnetic films were studied via MOKE and they were analyzed using TMR measurements. The results have been shown in the figure 4.31

TEM analysis of the junctions

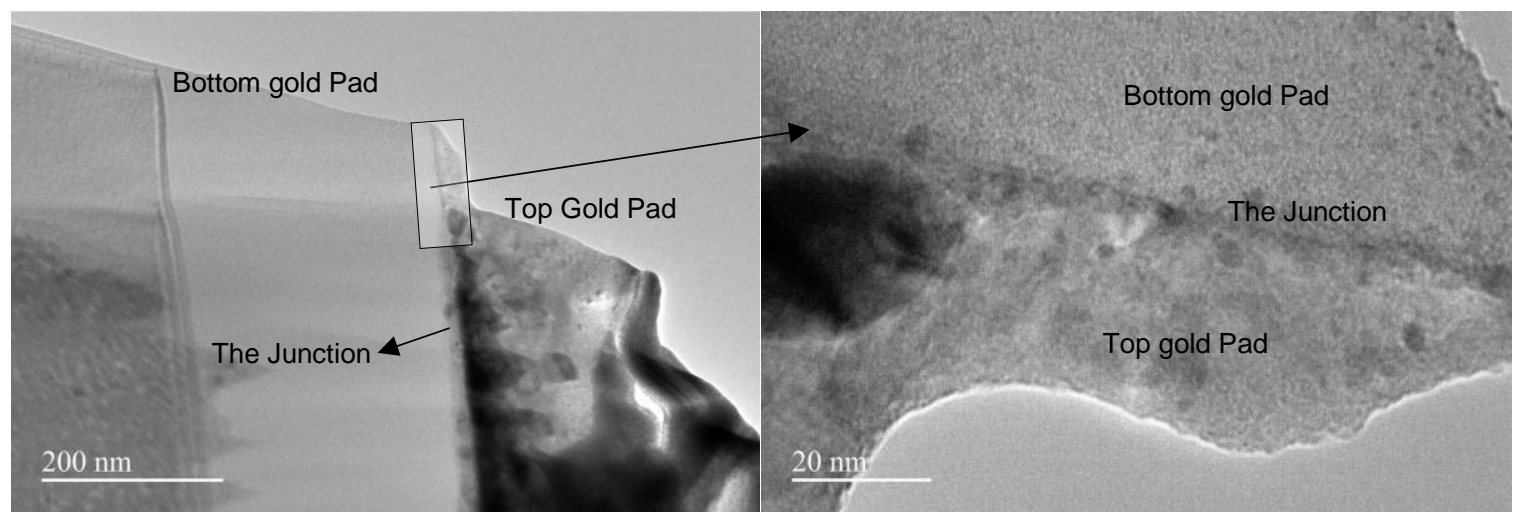

Figure 4.29. TEM image of a MTJ prepared by the FIB

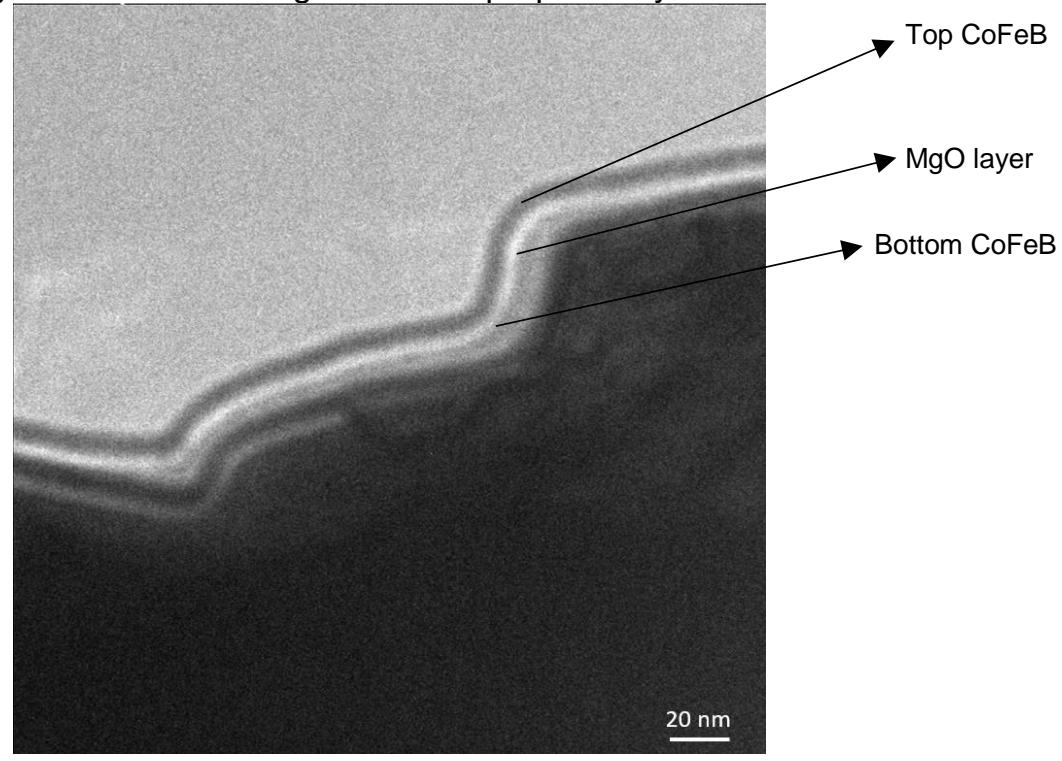

Figure 4.30. A high magnification TEM image of a MTJ showing different layers of the junction 

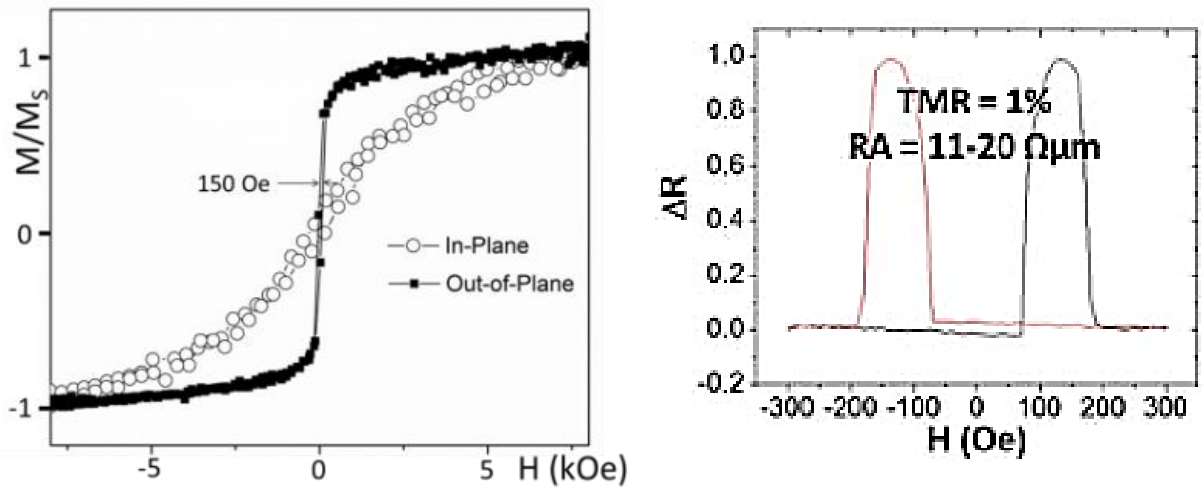

Figure 4.31. M-H loop of the fabricated pristine film on the left and TMR measurement on the right

A lock-in amplifier with a magnetic field perpendicular to the junction plane was used in order to carry out the magnetotransport measurements. A Kethley meters (current source and nano-voltmeter) was employed in order to do the I-V curve measurements. A focused magneto-optical Kerr effect (MOKE) setup in the polar Kerr rotation mode did M-H loops. First generation of MTJs
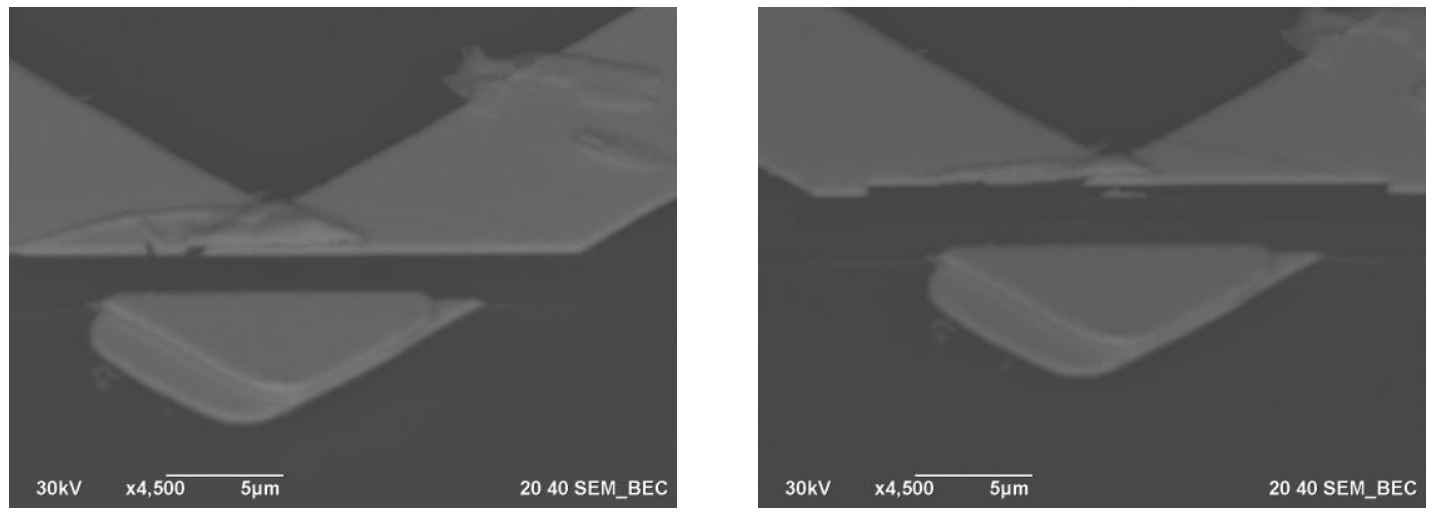

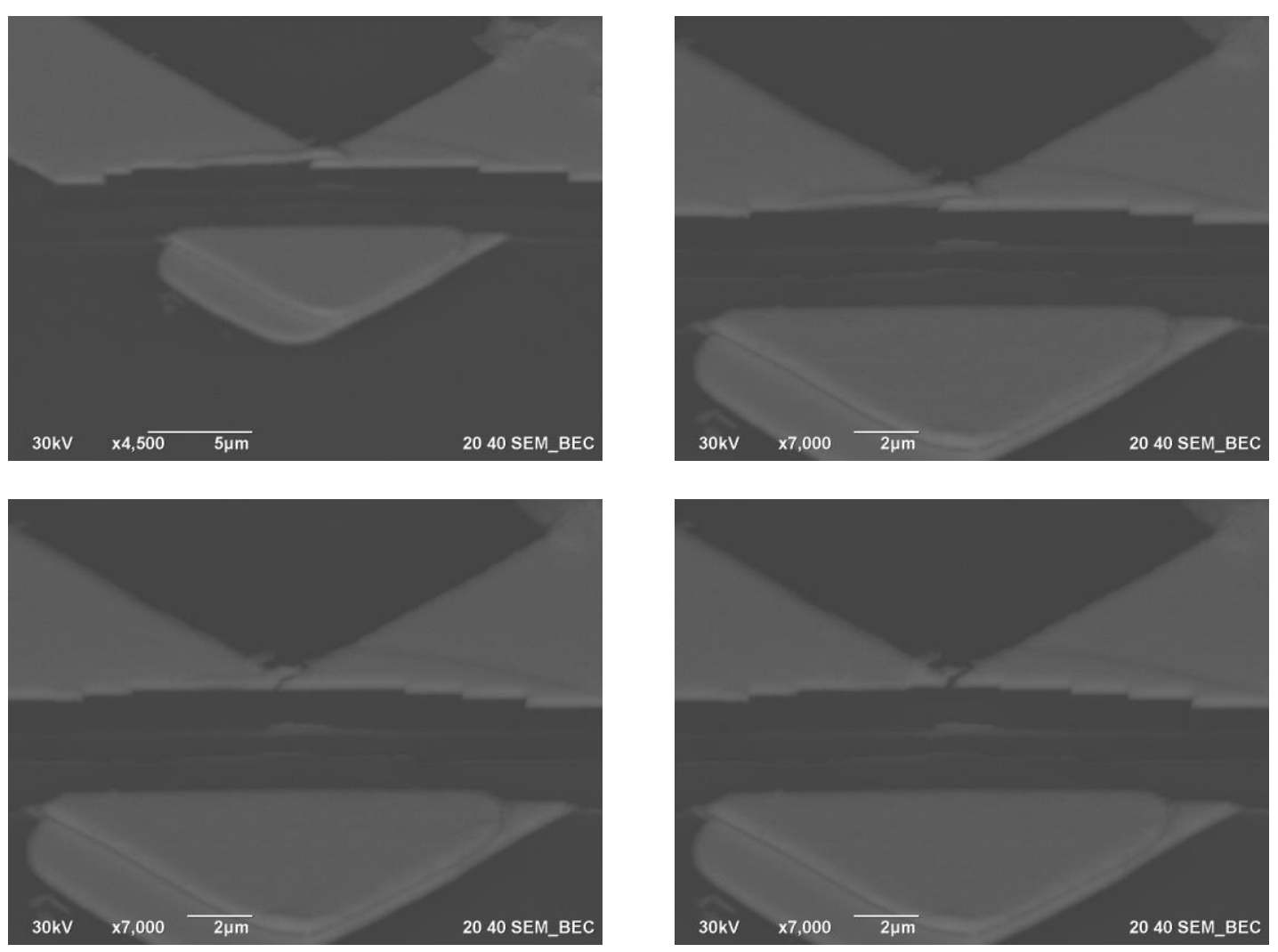

Figure 4.32. SEM image of a first generation MTJ in different steps of the milling

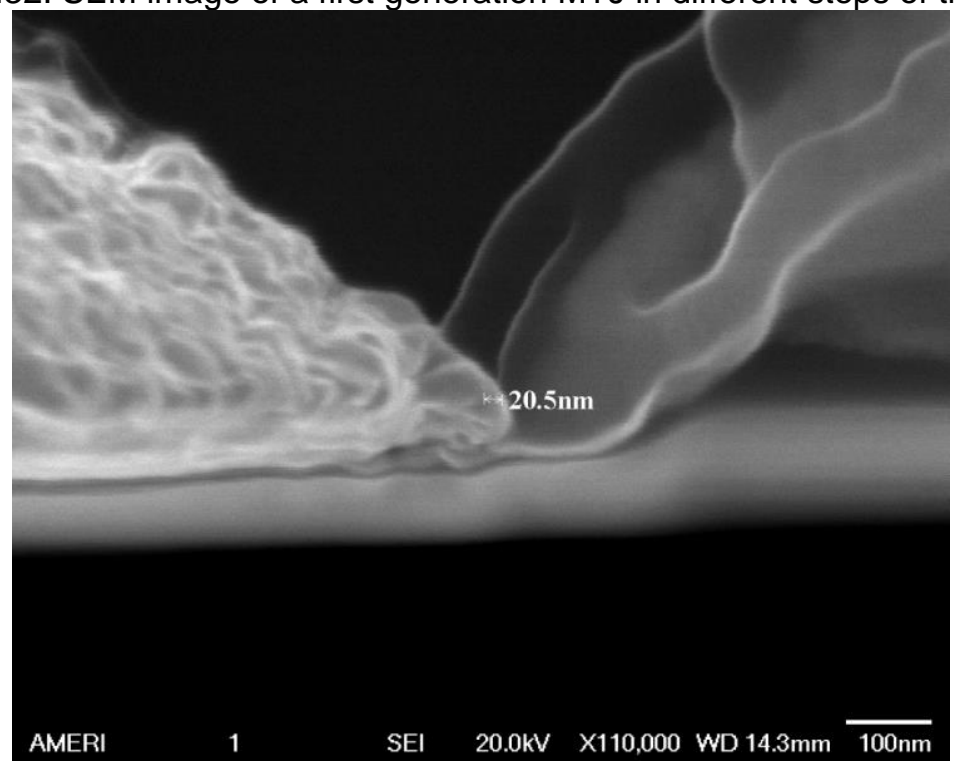

Figure 4.33. SEM image of the final MTJ 
Second generation of MTJs
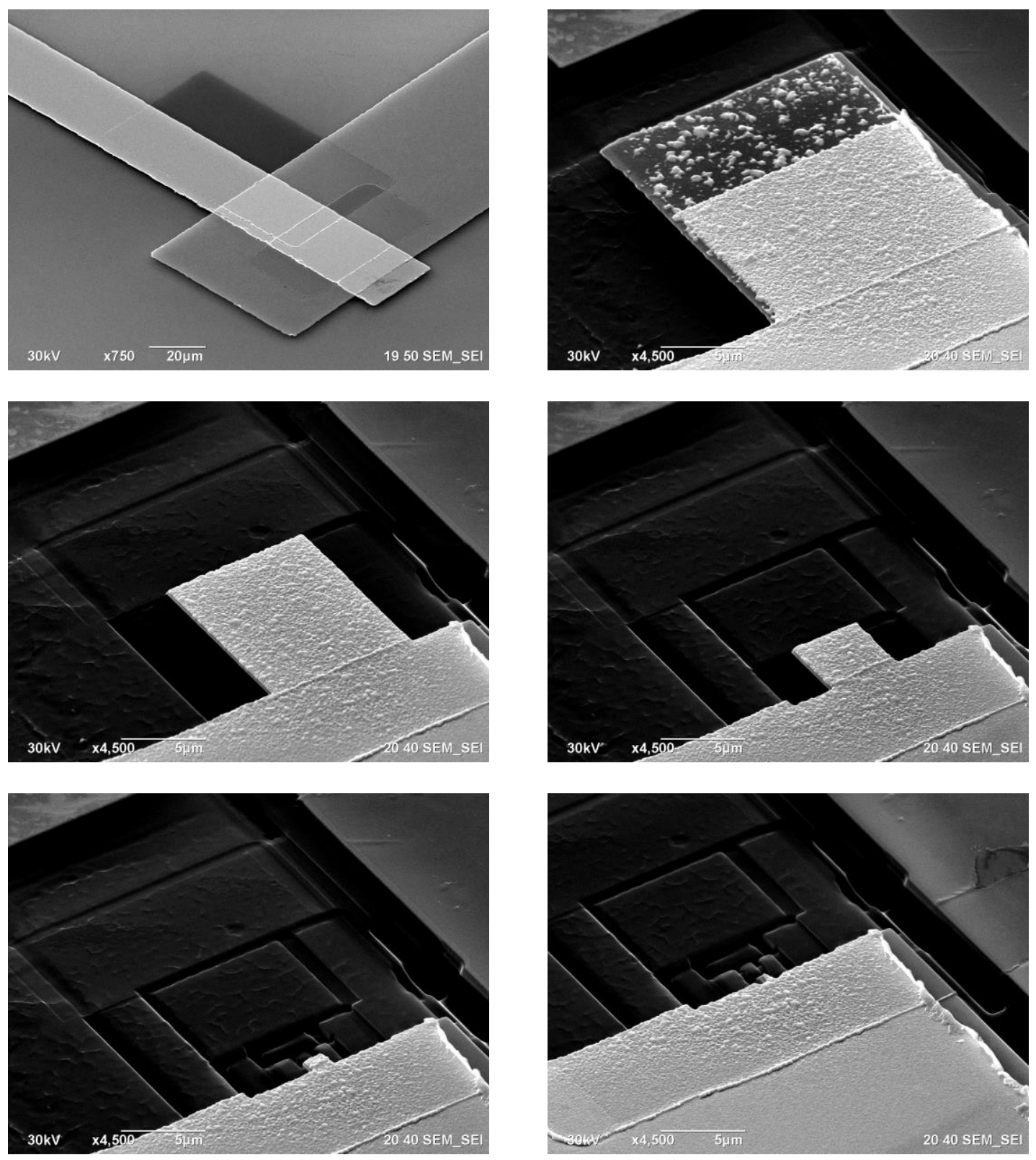

Figure 4.34. SEM image of a second generation MTJ in different steps of the milling process

After using $\mathrm{Ga}$ ions in order to make the structures as small as possible, a series of $\mathrm{He}$ and $\mathrm{Ne}$ ion millings were performed. Due to the limited resolution of Ga milling $(\sim 20 \mathrm{~nm}$, depending on the sample properties), Ga ion FIB is unable to yield the expected resolution 
on its own. For this reason it was decided that a series of complementary millings with $\mathrm{Ne}$ and He should follow the Ga millings in order for the junction to reach the appropriate size.

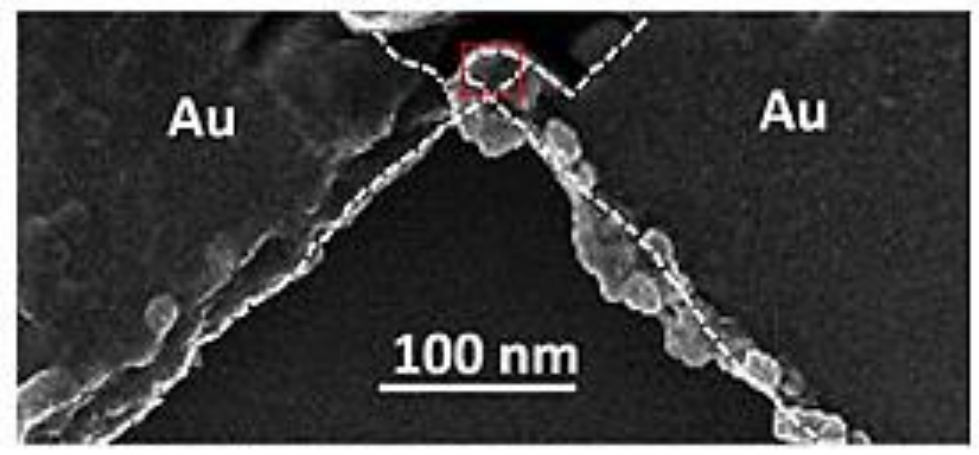

Figure 4.35. He ion image the MTJ (red box)

Also in some cases it is attractive to use a FIB for imaging instead of an SEM. Since, the ions have a much higher mass their effective wavelength is shorter and this leads to probe sizes as small as 3 to $5 \mathrm{~nm}$. However using a heavy ion such as a gallium for imaging has an important disadvantage. The small details are being sputtered while imaging and this puts a limitation on ultimate resolution of the imaging using a $\mathrm{Ga}$ ion beam. Helium ions have a largely reduced ability to sputter materials. Hence, the ultimate image resolution is much better as compared to using a heavier ion. This advantage along with a high brightness source results in a probe size of $\sim 0.3 \mathrm{~nm}$ that even outperforms an SEM in some applications [5].

The smallest fabricated device was approximately $5 \mathrm{~nm}$. Since the resolution of the SEM used for this analysis is limited, this value was also confirmed by estimating the resistance value. This value scales with the cross sectional area. Figure 4.36 shows the switching current and the magnetoresistance for the device. The measured switching current is approximately $0.6 \mu \mathrm{A}$. This value is comparable to the earlier measurements taken through the nanoprobe method, which was around $0.1 \mu \mathrm{A}$. The measured TMR ration is 
approximately $60 \%$, which is again comparable to the nanoprobe measurements which were around $30 \%$ for such a small device $[6,7]$.

Helium-Neon ion milling in Carl Zeiss

In a collaboration between FIU and Carl Zeiss a few samples were shipped to Carl Zeiss, Peabody MA for studying the effect of using different ions. Sample was first prepared with Ga-FIB to remove the majority of the excess gold contacts with $\sim 10 \mu \mathrm{m} \times 10 \mu \mathrm{m}$ area. Following the Ga-FIB preparation, neon ion beam was used to fabricate the $10 \mathrm{~nm} \times 10 \mathrm{~nm}$ junction. Due to the small dimension of the design, patterning was unsuccessful with the $10 \mathrm{~nm} \times 10 \mathrm{~nm}$ region being milled by the neon ion beam as well. Details of the patterning can be observed in the following figures
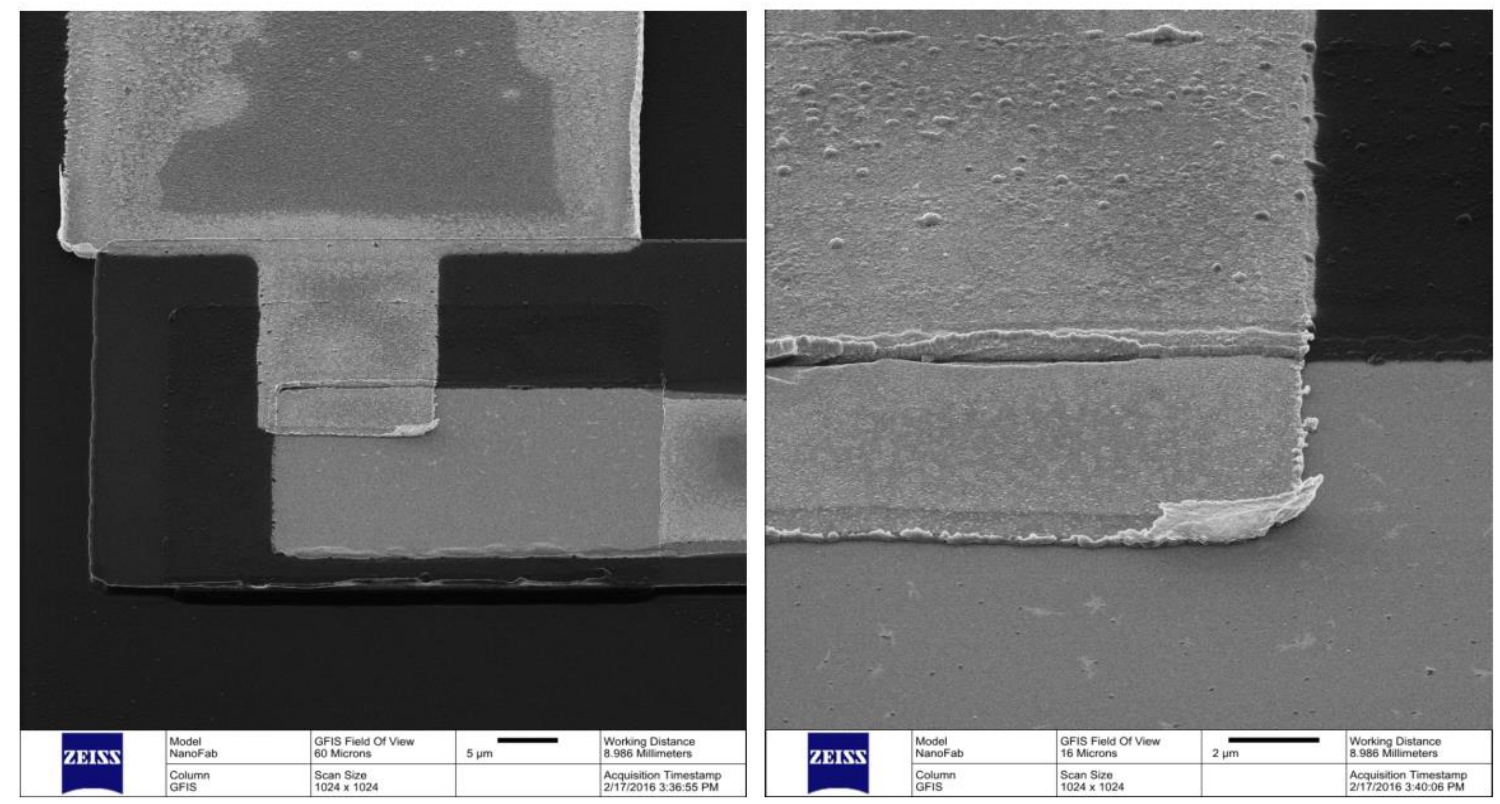

Figure 4.36. The junction before any fabrication work 


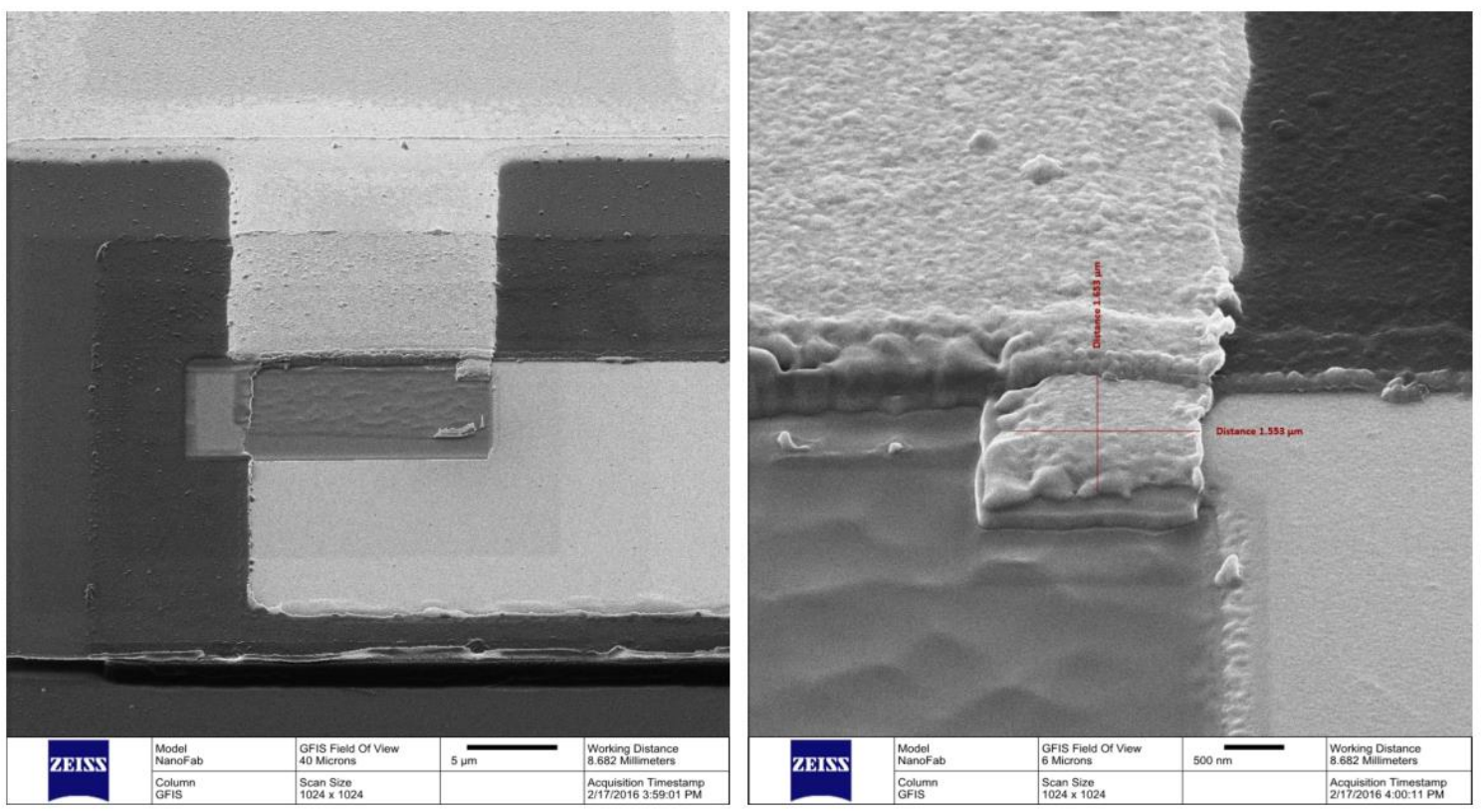

Figure 4.37. The junction after Ga fibbing
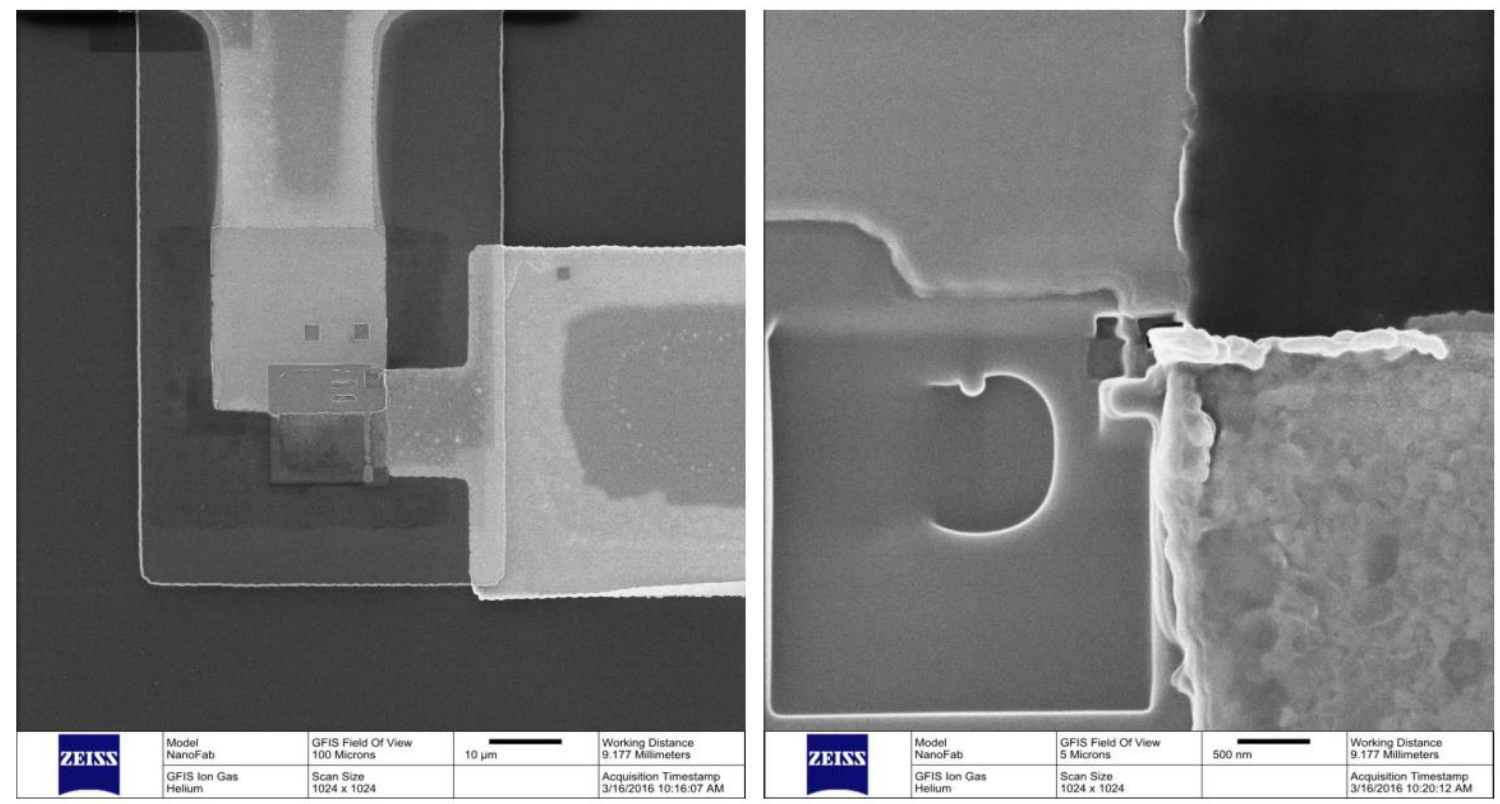

Figure 4.38. The junction after Ne patterning 
References:

1. van der Wouden, Els A., et al. "Approaches and methods in gene therapy for kidney disease." Journal of pharmacological and toxicological methods 50.1 (2004): 13-24.

2. Farquhar, Marilyn G., Steven L. Wissig, and George E. Palade. "Glomerular permeability I. Ferritin transfer across the normal glomerular capillary wall."The Journal of experimental medicine 113.1 (1961): 47-66.

3. Pease, Daniel C. "Fine structures of the kidney seen by electron microscopy." Journal of Histochemistry \& Cytochemistry 3.4 (1955): 295-308.

4. Alexis, Frank, et al. "Factors affecting the clearance and biodistribution of polymeric nanoparticles." Molecular pharmaceutics 5.4 (2008): 505-515.

5. Hill, Ray, John A. Notte, and Larry Scipioni. "Scanning helium ion microscopy." Advances in Imaging and Electron Physics 170 (2012): 65-148.

6. Hong, Jeongmin, et al. "The Physics of Spin-transfer Torque Switching in Magnetic Tunneling Junctions in Sub-10-nm Size Range."

7. Hong, Jeongmin, et al. "Energy-efficient spin-transfer torque magnetization reversal in sub-10-nm magnetic tunneling junction point contacts." Journal of nanoparticle research 15.4 (2013): 1-6. 
Recommendations for Future Work

Suggestions for future work can be classified in the following order:

\section{Nanomedicine}

\section{Including shorter time points}

Harvesting and studying the organs one hour and twenty-four hours after injection can yield valuable data about how much of the particles get discharged through digestive tract right after the injection.

\section{Including urine and feces samples}

A research has to be conducted in order to study how much of the particles get discharged by urine over time and how much of the particles get discharged by feces over time. This can provide a better understanding of the path the particles take and their metabolism in the blood stream. This study can be conducted both by EDS and backscattered electron microscopy and by employing differential scanning calorimetry.

\section{Simultaneous Thermal Analysis (TGA/DSC)}

Using SEM and EDS is a suitable method for the study of the particle clusters in the tissue. SEM and EDS can identify the exact location of the particle clusters in each part of the tissue. However, for the case of urine and feces, detecting the exact location of the particle is not of interest anymore. It is more important to know exactly how much of the particles are leaving the body at each point of time. Another issue with SEM study of the tissue is that these types of analysis yield two-dimensional information. All the analysis is taking place on section of a tissue that might or might not represent the distribution in that organ. For this reason, a lot of information from different regions of different sections of an organ needs to be gathered and averaged. This ensures that the results are not biased. 
Differential scanning calorimetry (DSC) is a thermoanalytical method that is often used to calculate reaction energy and temperature, melting temperature, heat of fusion, latent heat of melting, crystalline phase transition temperature and energy, oxidation induction times, and specific heat or heat capacity. DSC computes the energy that is absorbed or released by the sample when it is cooled or heated and produces qualitative and quantitative data on endothermic and exothermic processes. Thermogravimetric analysis (TGA) is a technique that measures the change in physical and chemical properties of materials as a function of temperature. TGA can provide data about physical and chemical phenomena including vaporization, sublimation and solid gas reactions like decomposition and oxidation. TGA is often used to study the materials that exhibit mass loss or gain because of decomposition and oxidation. One of the common applications of TGA which is related to this study is determination of inorganic content in a sample. Simultaneous application of DSC and TGA can provide useful information about the inorganic content of the bulk samples. Since for the analysis of feces and urine there is no need for site-specific detection the samples can be analyzed by DSC/TGA and since the bulk sample can be loaded the results are comparatively accurate and reliable. This method is cost effective and can also be used for other organs along with the microscopy analysis in order to collect complementary data. It should be noted that inorganic elements exist in different organs, feces and urine. Hence, there should be some runs with control samples for eliminating the effect of these elements.

\section{Using Inductively coupled plasma mass spectrometry (ICP-MS)}

Inductively coupled plasma mass spectrometry (ICP-MS) is capable of detecting metals and non-metals at concentrations as low as one part in $10^{15}$. This type of mass spectroscopy ionizes the sample with inductively coupled plasma and then exploits a mass spectrometer to separate and quantify those ions. In order to use this method the 
sample has to introduced to the plasma as aerosol. Hence, the bulk sample should be digested in a proper solvent. This process has a rather high precision but it is not straightforward and might be expensive.

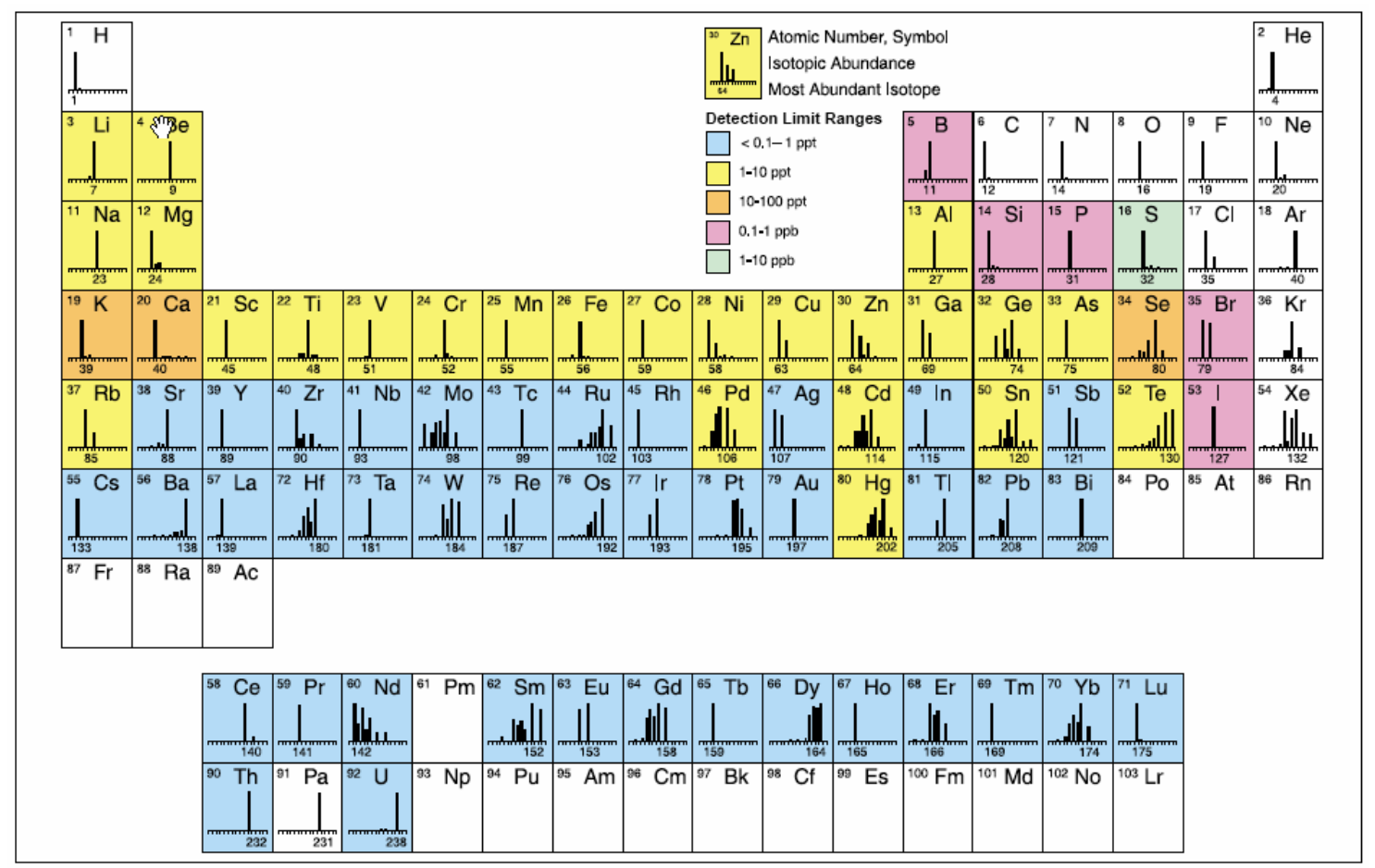

Figure 5.1. Approximate detection capabilities of the ICP-MS method. (Courtesy of PerkinElmer, Inc.)

\section{Nanoelectronics}

\section{EDS assisted TEM study of the samples}

Each layer of the junction that is going to be sputter deposited has a significant effect on the tunneling properties of the final junction. Hence, it is of utmost importance to characterize each layer, confirm its uniform deposition and measure the thickness of each layer to ensure that the junction that is going to be trimmed by fibbing, passes the magnetic, tunneling and resistance requirements. One of the most effective ways to characterize the magnetic tunneling junctions is TEM analysis. This characterization 
method has been employed throughout this study for several samples but the study could not be confirmed by EDS analysis of the TEM samples. EDS analysis of TEM samples inside the microscope enables nanometer resolution study of each layer. It can be employed to confirm uniform deposition of the deposited layers and is going to be an important part of the study for the future calibration and characterization of the MTJs.

\section{Incorporating nanoparticles in the new junctions}

The nanoparticle-based MTJs show the potential for low switching current and extremely high magnetoresistance ( $1000 \%)$ due to the magnetically affected Coulomb blockade in a single electron transport at room temperature. The structures that show promise are sub-10-nm MTJs trimmed via FIB, and MTJ with nanoparticles sandwiched between two magnetic layers (standard CoFeB material used in Spin transfer torque junctions). The nanoparticles are going to be separated from the magnetic layers by thin $0.3 \mathrm{~nm} \mathrm{MgO}$ layers. Nanoparticles in the magnetic sandwich are made of $\mathrm{CoFe} 2 \mathrm{O} 4$ ferrimagnetic material with a very high anisotropy to ensure thermal stability

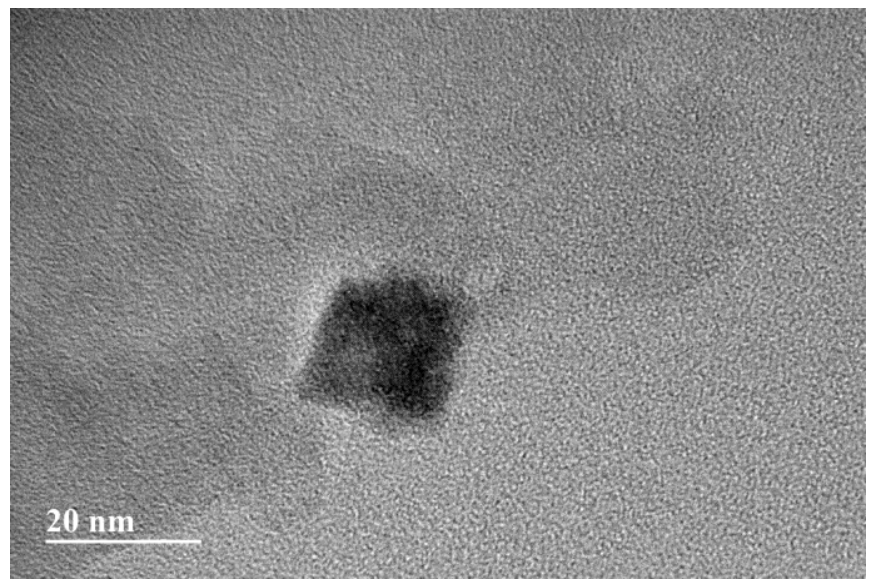

Figure 5.2. TEM image of a CoFe2O4 nanoparticle 


\section{Ion implantation study}

In order to study the effect of fibbing and ion implantation on magnetoresistance and electrical properties of the junction a comprehensive study of ion implantation should be carried out in the future.

\section{Calibration of neon and helium sources and using glass wafers instead of silicon}

It was observed that overexposing the sample to helium might cause nanometer-scale deformation of the sample. Trapped helium atoms merge into nanometer-scale bubbles that form under the exposed area cause this deformation of the sample. Using glass wafers instead of silicon wafers can reduce this problem considerably. Also on several occasions, the sample was lost due to the unstable neon source emission. In order to have repeatable fabrication exposure times for helium and neon should be carefully calibrated in the future. 
VITAE

ALI HADJIKHANI

2004-2009

BSc., Physics

Amirkabir University of Technology

Tehran, Iran

$2010-2012$

MSc., Materials Science and Engineering

Florida International University

Miami, Florida

2012-2016

$\mathrm{PhD}$, Electrical Engineering

Florida International University

Miami, Florida

\section{PUBLICATIONS AND PRESENTATIONS}

1- Hadjikhani, A., Rodzinski, A., and Khizroev, S., Biodustribution study of magneto electric nanoparticles used for anticancer drug delivery. In preparation for Nano Letters

2- Hadjikhani, A., Stone, M., Guduru, R., and Khizroev, S., Using MgO thickness to control the interplay between exchange and anisotropy energies in spin transfer torque devices.to be submitted to IEEE Trans Magn,

3- Rodzinski, A., Guduru, R., Liang, P., Hadjikhani, A., Stewart, T., Stimphil, E., Runowicz, C., Cote, R., Altman, N., Datar, R. and Khizroev, S., 2016. Targeted and controlled anticancer drug delivery and release with magnetoelectric nanoparticles. Scientific reports, 6.

4- J. Hong, A. Hadjikhani, M. Stone, F. Allen, V. Safonov, P. Liang, J. Bokor, and S. Khizroev, "The physics of spin-transfer torque switching in magnetic tunneling junctions in sub-10-nm size range," IEEE Trans. Magn. accepted (2016)

5- Stone, M., Hong, J., Hadjikhani, A., Guduru, R., Manoussakis, A., Stimphil, E., Luongo, K., Liang, P., Safonov, V., Bokor, J. and Khizroev, S., 2015, October. 
Anomalous properties of sub-10-nm magnetic tunneling junctions. In Energy Efficient Electronic Systems (E3S), 2015 Fourth Berkeley Symposium on (pp. 13). IEEE.

6- Guduru, R., Liang, P., Hong, J., Rodzinski, A., Hadjikhani, A., Horstmyer, J., Levister, E. and Khizroev, S., 2015. Magnetoelectric'spin'on stimulating the brain. Nanomedicine, 10(13), pp.2051-2061.

7- Rodzinski, A., Hadjikhani, A., Stewart, T., Stimphil, E., Guduru, R., Liang, P., Runowicz, C. and Khizroev, S., 2016. Abstract B47: A novel mechanism for fieldcontrolled high-specificity targeted anticancer drug delivery and on-demand release using magnetoelectric nanoparticles. Cancer Research, 76(3 Supplement), pp.B47-B47.

8- Lahiri, D., Hadjikhani, A., Zhang, C., Xing, T., Li, L.H., Chen, Y. and Agarwal, A., 2013. Boron nitride nanotubes reinforced aluminum composites prepared by spark plasma sintering: Microstructure, mechanical properties and deformation behavior. Materials Science and Engineering: A, 574, pp.149-156

9- Karbasi, A., Hadjikhani, A., Hrubiak, R., Durygin, A. and Jones, K., 2013. High Temperature Interactions in Platinum/Alumina System. Processing and Properties of Advanced Ceramics and Composites V: Ceramic Transactions, Volume 240, pp.45-53.

10- A. Hadjikhani, J. Chen, S. Das, W. Choi, "Raman Spectroscopy of Graphene and Plasma treated Graphene Under High Pressure" published in the proceeding TMS annual meeting 2012

11- A. Hadjikhani, J. Chen, S. Das, W. Choi, "Raman Spectroscopy of Graphene Under High Pressure" Compres, Williamsburg 2011 